\title{
Rayleigh-Bénard convection: The container shape matters
}

\author{
Olga Shishkina $~_{*}^{*}$ \\ Max Planck Institute for Dynamics and Self-Organization, 37077 Göttingen, Germany
}

(Received 6 July 2021; accepted 8 September 2021; published 28 September 2021)

To study turbulent thermal convection, one often chooses a Rayleigh-Bénard flow configuration, where a fluid is confined between a heated bottom plate, a cooled top plate of the same shape, and insulated vertical sidewalls. When designing a Rayleigh-Bénard setup, for specified fluid properties under Oberbeck-Boussinesq conditions, the maximal size of the plates (diameter or area), and maximal temperature difference between the plates, $\Delta_{\max }$, one ponders: Which shape of the plates and aspect ratio $\Gamma$ of the container (ratio between its horizontal and vertical extensions) would be optimal? In this article, we aim to answer this question, where under the optimal container shape, we understand such a shape, which maximizes the range between the maximal accessible Rayleigh number and the critical Rayleigh number for the onset of convection in the considered setup, $\mathrm{Ra}_{c, \Gamma}$. First we prove that $\operatorname{Ra}_{c, \Gamma} \propto\left(1+c_{u} \Gamma^{-2}\right)\left(1+c_{\theta} \Gamma^{-2}\right)$, for some $c_{u}>0$ and $c_{\theta}>0$. This holds for all containers with no-slip boundaries, which have a shape of a right cylinder, whose bounding plates are convex domains, not necessarily circular. Furthermore, we derive accurate estimates of $\mathrm{Ra}_{c, \Gamma}$, under the assumption that in the expansions (in terms of the Laplace eigenfunctions) of the velocity and reduced temperature at the onset of convection, the contributions of the constant-sign eigenfunctions vanish, both in the vertical and at least in one horizontal direction. With that we derive $\operatorname{Ra}_{c, \Gamma} \approx(2 \pi)^{4}\left(1+c_{u} \Gamma^{-2}\right)\left(1+c_{\theta} \Gamma^{-2}\right)$, where $c_{u}$ and $c_{\theta}$ are determined by the container shape and boundary conditions for the velocity and temperature, respectively. In particular, for circular cylindrical containers with no-slip and insulated sidewalls, we have $c_{u}=j_{11}^{2} / \pi^{2} \approx 1.49$ and $c_{\theta}=\left(\widetilde{j}_{11}\right)^{2} / \pi^{2} \approx 0.34$, where $j_{11}$ and $\tilde{j}_{11}$ are the first positive roots of the Bessel function $J_{1}$ of the first kind or its derivative, respectively. For parallelepiped containers with the ratios $\Gamma_{x}$ and $\Gamma_{y}$, $\Gamma_{y} \leqslant \Gamma_{x} \equiv \Gamma$, of the side lengths of the rectangular plates to the cell height, for no-slip and insulated sidewalls we obtain $\operatorname{Ra}_{c, \Gamma} \approx(2 \pi)^{4}\left(1+\Gamma_{x}^{-2}\right)\left(1+\Gamma_{x}^{-2} / 4+\Gamma_{y}^{-2} / 4\right)$. Our approach is essentially different to the linear stability analysis, however, both methods lead to similar results. For $\Gamma \lesssim 4.4$, the derived $\mathrm{Ra}_{c, \Gamma}$ is larger than Jeffreys' result $\mathrm{Ra}_{c, \infty}^{J} \approx 1708$ for an unbounded layer, which was obtained with linear stability analysis of the normal modes restricted to the consideration of a single perturbation wave in the horizontal direction. In the limit $\Gamma \rightarrow \infty$, the difference between $\operatorname{Ra}_{c, \Gamma \rightarrow \infty}=(2 \pi)^{4}$ for laterally confined containers and Jeffreys' $\mathrm{Ra}_{c, \infty}^{J}$ for an unbounded layer is about $8.8 \%$. We further show that in Rayleigh-Bénard experiments, the optimal rectangular plates are squares, while among all convex plane domains, circles seem to match the optimal shape of the plates. The optimal $\Gamma$ is independent of $\Delta_{\max }$ and of the fluid properties. For the adiabatic sidewalls, the optimal $\Gamma$ is slightly smaller than $1 / 2$ (for cylinder, about 0.46 ), which means that the intuitive choice of $\Gamma=1 / 2$ in most Rayleigh-Bénard experiments

\section{*Olga.Shishkina@ds.mpg.de}

Published by the American Physical Society under the terms of the Creative Commons Attribution 4.0 International license. Further distribution of this work must maintain attribution to the author(s) and the published article's title, journal citation, and DOI. Open access publication funded by the Max Planck Society. 
is right and justified. For the given plate diameter $D$ and maximal temperature difference $\Delta_{\max }$, the maximal attainable Rayleigh number range is about 3.5 orders of magnitudes smaller than the order of the Rayleigh number based on $D$ and $\Delta_{\max }$. Deviations from the optimal $\Gamma$ lead to a reduction of the attainable range, namely, as $\log _{10}(\Gamma)$ for $\Gamma \rightarrow 0$ and as $\log _{10}\left(\Gamma^{-3}\right)$ for $\Gamma \rightarrow \infty$. Our theory shows that the relevant length scale in Rayleigh-Bénard convection in containers with no-slip boundaries is $\ell \sim D / \sqrt{\Gamma^{2}+c_{u}}=H / \sqrt{1+c_{u} / \Gamma^{2}}$. This means that in the limit $\Gamma \rightarrow \infty, \ell$ equals the cell height $H$, while for $\Gamma \rightarrow 0$, it is rather the plate diameter $D$.

DOI: 10.1103/PhysRevFluids.6.090502

\section{INTRODUCTION}

One of the very few advantages of a virtual format of the 73rd Annual Meeting of the APS Division of Fluid Dynamics in virtual Chicago was the possibility to conduct an audience survey. An anonymous poll that was launched at the very beginning of my talk and continued for about one or two minutes had a single polling statement: "Rayleigh-Bénard convection in a cylinder for $\mathrm{Ra}=10^{20}$ and $\mathrm{Pr}=1$ is turbulent" with the following response options: (1) "yes," (2) "no," and (3) "I don't know." The polling report stated that 52\% of participants answered "yes," 7\% replied "no," and $41 \%$ did not know whether convection at these values of the Rayleigh number Ra and Prandtl number Pr would be turbulent or not. Of course, the results of this poll should not be taken seriously, as it was conceived and conducted for entertainment; however, the lack of unanimity in the responses of the participants is quite noteworthy. If I would have taken part in the poll, I also would choose the third response option, because information about the control parameters $\mathrm{Ra}$ and $\mathrm{Pr}$ is not sufficient to give a definite yes or no: even in the simplest case of convection within the Oberbeck-Boussinesq approximation [1,2], where the fluid properties are assumed to be independent of temperature and pressure, we need to know at least the diameter-to-height aspect ratio $\Gamma$ of the cylindrical container, in order to be able to judge on the flow state, even for that large values of Ra. In this article, we will discuss why the aspect ratio of the container, along with the other control parameters, $\mathrm{Ra}$ and $\mathrm{Pr}$, plays a very important role in Rayleigh-Bénard [3-5] experiments and numerical simulations (see [6-9]).

Among other natural convection systems, like horizontal convection [10-14] or vertical convection (see, e.g., [15-20]), Rayleigh-Bénard convection (RBC) [3-5] (see also [6-8,21,22]), being of particular importance in astrophysical and geophysical systems and in many industrial applications, has been the most studied paradigmatic buoyancy-driven flow. Rayleigh-Bénard convection is a fluid motion that occurs in a fluid layer confined between two isothermal horizontal surfaces of different temperatures when a certain critical Rayleigh number is exceeded. In the case of an infinite fluid layer and no-slip boundary conditions at the plates, this critical Rayleigh number for the onset of convection $\mathrm{Ra}_{c, \infty}^{J}$ is about 1708, as it was calculated by Jeffreys [23] and Harris and Reid [24] with the linear stability analysis of the normal modes restricted to the consideration of a single perturbation wave in the horizontal direction.

Although the onset of convection is known to be independent of Pr, the Prandtl number of the fluid influences the flow dynamics and heat transport in larger Ra regimes. Thus, the main dimensionless control parameters in Rayleigh-Bénard convection are the Rayleigh number Ra and the Prandtl number Pr, which are defined, respectively, as

$$
\mathrm{Ra} \equiv \alpha g \Delta H^{3} /(\kappa \nu) \quad \text { and } \quad \operatorname{Pr} \equiv \nu / \kappa
$$

where $\alpha$ is the isobaric thermal expansion coefficient, $v$ the kinematic viscosity, $\kappa$ the thermal diffusivity, $g$ the gravitational acceleration, $H$ the depth of the fluid layer (or the height of the Rayleigh-Bénard cell), and $\Delta \equiv T_{b}-T_{t}$ is the temperature difference between the hot bottom surface of the fluid layer and its cold top surface. 
The turbulent state of Rayleigh-Bénard convection, which develops for very large Ra, is the most relevant in many astrophysical and geophysical applications and at the same time is the most challenging for experimental and numerical studies. Accurate numerical simulations for high $\mathrm{Ra}$ require very fine grids in space and time and, therefore, extremely huge and not always feasible computational resources. On the other hand, for a fixed fluid and a fixed pressure used in a RayleighBénard experiment under Oberbeck-Boussinesq conditions, increasing Ra requires either $\Delta$ or $H$ to be increased. Therefore, achieving possibly large $\mathrm{Ra}$ in experiments requires huge setups, with possibly large height $H$, since the maximal variation of the temperature difference between the bottom and top plates, i.e., the maximal $\Delta=\Delta_{\max }$, is quite restricted, especially if one aims to conduct experiments under almost Oberbeck-Boussinesq conditions. In an effort to achieve very large values of $\mathrm{Ra}$, it becomes tempting to carry out experiments and simulations of RayleighBénard convection for as slender convection cells as possible, which in terms of dimensionless numbers means to use convection cells with as small aspect ratio $\Gamma$,

$$
\Gamma \equiv D / H
$$

as possible. Here $D$ is a horizontal extension of the convection cell. In the case of a cylindrical cell, $D$ is the diameter of the cylinder, while in the case of a parallelepiped cell, $D$ usually denotes the length of the longest side of a rectangular plate. Thus, next to $\mathrm{Ra}$ and $\mathrm{Pr}$, another control parameter in the system is $\Gamma$. While aiming to achieve possibly large Ra in a certain Rayleigh-Bénard setup, one usually assumes that one would cover a broader Ra range, measured in orders of magnitude of Ra values, starting from the critical Rayleigh number for the onset of convection and up to the maximal Rayleigh number that can be achieved in this experimental setup. But does an increase in the height of the container always lead to an expansion of the parameter range that can be studied using this cell (measured in orders of magnitude of Ra)? With this respect, what would be the optimal choice of the cell height, for given heated and cooled plates? Which shape should the plates have - a circle, a square, or maybe an elongated rectangle - to maximize the Ra range that can be studied in a Rayleigh-Bénard experiment using these plates? And what is, in general, the role of the aspect ratio $\Gamma$ in Rayleigh-Bénard convection, close to the onset of convection and up to the fully developed turbulent flow?

In this article, we intend to answer these questions for the case of Oberbeck-Boussinesq Rayleigh-Bénard convection and discuss further related topics.

The paper is organized as follows. In Sec. II we will discuss the dependence of the onset of convection on the container shape and will derive estimates of the critical Rayleigh number for the onset of convection in cylindrical and parallelepiped domains as a function of the domain aspect ratio. Section III is devoted to the discussion of the optimality of the shape of the Rayleigh-Bénard cell, the form of the cell plates, and the optimal cell height. The optimality of the shape of the cylindrical and parallelepiped domains will be formulated in terms of their aspect ratios. In Sec. IV we will derive the relevant length scale in Rayleigh-Bénard convection and show the way how, with that length scale, one can collapse all data for different $\Gamma$ on a master curve. In Sec. V we will discuss further applications of the developed ansatz to thermally driven flows. Finally, in the last Sec. VI, we will summarize results and give an outlook.

\section{ONSET OF CONVECTION}

In order to determine the optimal container shape that provides a maximum range of Rayleigh numbers, starting from the onset of convection, we need to know how the onset of convection depends on the container shape. For standard geometries like parallelepiped or circular cylinder we also need simple but sufficiently accurate formulas to estimate the critical Rayleigh number for the onset of convection as a function of the container aspect ratio $\Gamma$.

The onset of convection has been studied quite intensively in the past, mainly for an infinite fluid layer (theoretically) and for containers of large aspect ratios (experimentally) (see [6,21,25-27]). Thus, under assumption that in the horizontal directions, the first unstable mode is represented by a 
single wave $\sim \exp \left(i k_{x} x+i k_{y} y+\sigma t\right)$, the onset of convection in an infinite layer bounded by no-slip heated bottom and cooled top plates should occur at a critical $\mathrm{Ra}_{c, \infty}^{J} \approx 1708$, according to Jeffreys [23] and Reid and Harris [24]. Due to the above assumption, this estimate can be considered as an upper bound of the critical Rayleigh number for the onset of bulk convection in the limit $\Gamma \rightarrow \infty$.

For a laterally confined container, the critical $\mathrm{Ra}_{c, \Gamma}$ gets generally larger with decreasing $\Gamma$ [28-38]. Charlson and Sani [28] considered cylindrical containers of different aspect ratios, from 1 to 16 , and obtained that the upper bounds for the critical Rayleigh number for the onset of axisymmetric modes of convection decrease with growing $\Gamma$. Similar tendency was reported by Charlson and Sani [29] for small aspect ratios $\Gamma<1$ as well. They also reported that with decreasing $\Gamma$, a transition in the marginal dynamic state from an axisymmetric state to an asymmetric state happens at $\Gamma \approx 1.6$ for adiabatic lateral walls and at $\Gamma \approx 1.2$ for conducting sidewalls. These results were in agreement with the previous experiments by Ostroumov [39] and later were also confirmed by Hebert et al. [36].

Numerical solutions of the linearized perturbation equations for a cylindrical container by Catton and Edwards [31] showed that the critical Rayleigh number for the first mode of convection for a conducting sidewall is larger than for an adiabatic sidewall, and this difference is larger for smaller aspect ratio of the container. For the limit $\Gamma \rightarrow 0$ they also proposed the scaling $\operatorname{Ra}_{c, \Gamma} \sim \Gamma^{-4}$ for the onset Rayleigh number.

Buell and Catton [32] conducted similar analysis as Charlson and Sani [29,29] but for arbitrary conductivity of the sidewall. They came to similar conclusions; however, more accurate simulations showed that the critical Rayleigh numbers for the asymmetric modes are significantly ( $20 \%)$ lower than previously reported. Thus, for any boundary conditions at the sidewall, the first asymmetric mode is the least stable for all small aspect ratios up to about $\Gamma \leqslant 1.6$. At large aspect ratios, the critical flow state alternates between the axisymmetric and asymmetric modes. Recent simulations by Yu et al. [38] for integer values of $\Gamma, 6 \leqslant \Gamma \leqslant 20$, confirmed this fact and also showed that even and odd values of $\Gamma$ have different preferable modes.

In this section, starting from the governing equations, we will recollect some relevant relations and then will derive a general estimate of a critical Rayleigh number for the onset of convection in any geometry and finally will provide quite accurate estimates of the critical Rayleigh number for the onset of convection in cylindrical and parallelepiped containers.

\section{A. Towards a critical Rayleigh number for the onset of convection}

Within the Oberbeck-Boussinesq approximation, the Rayleigh-Bénard flow is described by the following system of the governing Navier-Stokes, energy, and continuity equations for the velocity $\mathbf{u}$, temperature $T$, and the kinematic pressure $p$ :

$$
\begin{gathered}
\partial_{t} \mathbf{u}+\mathbf{u} \cdot \nabla \mathbf{u}+\nabla p=\nu \nabla^{2} \mathbf{u}+\alpha g T \mathbf{e}_{z} \\
\partial_{t} T+\mathbf{u} \cdot \nabla T=\kappa \nabla^{2} T \\
\nabla \cdot \mathbf{u}=0 .
\end{gathered}
$$

Here $\mathbf{e}_{z}$ is a unit vector pointing upwards. The boundary conditions for the velocity are no-slip at all walls. The temperature equals $T_{b}$ at the bottom plate $(z=0)$ and $T_{t}$ at the top plate $(z=H)$. The sidewalls are adiabatic, $\partial T / \partial \mathbf{n}=0$. (Later we will also discuss conducting sidewalls, but our main focus here and derivations are for the insulated walls.)

The main response characteristic of a Rayleigh-Bénard flow is the dimensionless heat transport, which is known as the Nusselt number:

$$
\mathrm{Nu} \equiv \frac{\left\langle u_{z} T\right\rangle_{z}-\kappa \partial_{z}\langle T\rangle_{z}}{\kappa \Delta / H}
$$


where $\langle\cdot\rangle_{z}$ denotes the average in time and over a horizontal cross section at height $z$ from the bottom. Averaging Eq. (6) for $z \in[0 ; H]$ one obtains

$$
\mathrm{Nu}=\frac{H}{\kappa \Delta}\left\langle u_{z} T\right\rangle+1,
$$

where $\langle\cdot\rangle$ denotes the time and volume average.

For the mean kinetic energy dissipation rate $\epsilon_{u}$ and the thermal dissipation rate $\epsilon_{\theta}$,

$$
\begin{aligned}
\epsilon_{u} & =v\left\langle(\nabla \mathbf{u})^{2}\right\rangle, \\
\epsilon_{\theta} & =\kappa\left\langle(\nabla T)^{2}\right\rangle,
\end{aligned}
$$

where $(\nabla \mathbf{u})^{2} \equiv\left(\nabla u_{x}\right)^{2}+\left(\nabla u_{y}\right)^{2}+\left(\nabla u_{z}\right)^{2}$, we also recall the following well-known exact relations [40-42]:

$$
\begin{gathered}
\epsilon_{u}=\alpha g\left\langle u_{z} T\right\rangle, \\
\epsilon_{u}=\frac{\nu^{3}}{H^{4}}(\mathrm{Nu}-1) \frac{\mathrm{Ra}}{\mathrm{Pr}^{2}}, \\
\epsilon_{\theta}=\frac{\kappa \Delta^{2}}{H^{2}} \mathrm{Nu},
\end{gathered}
$$

which one obtains directly from the governing equations (3)-(5) and the boundary conditions.

Here we will derive a general form of the dependence of the critical Rayleigh number for the onset of convection, as a function of the container aspect ratio $\Gamma$. The approach we consider here is different to the linear stability analysis [25,43], but as we will see later, it allows us to derive simple but sufficiently accurate analytical estimates for the case of classical geometries of the RayleighBénard container, like a cylinder or a parallelepiped. Our approach is rather a variational one and, in contrast to the linear stability analysis, assumes neither vanishing contributions of the nonlinear terms in the governing equations, nor separation of variables in the horizontal and vertical directions for the flow components at the onset of convection, nor the same structure in the horizontal directions ( $x$ and $y$ ) of the velocity components and of the deviation of the temperature from the linear profile. Note that the latter assumption is quite restrictive in the case of confined containers with no-slip conditions for the velocity and adiabatic for the temperature: This combination of the boundary conditions actually excludes the legitimacy of reducing the stability analysis to the consideration of a single mode in the horizontal directions [in a form like $\sim \exp \left(i k_{x} x+i k_{y} y+\sigma t\right)$ ].

We start to proceed in quite a standard way (see, e.g., [44]) and decompose the temperature field into a linear function $T_{l}(z)$, that satisfies the boundary condition at the plates, and the residual function $\theta$ that vanishes at the plates:

$$
T \equiv T_{l}+\theta, \quad T_{l}(z) \equiv T_{b}-(z / H) \Delta .
$$

Since in a confined geometry $\left\langle u_{z}\right\rangle_{z}=0$ holds for any $z$, from this and Eq. (13) one obtains $\left\langle u_{z} T\right\rangle_{z}=$ $\left\langle u_{z} \theta\right\rangle_{z}$ for any $z$, which after integration over the cell height, $z \in[0 ; H]$, yields

$$
\left\langle u_{z} T\right\rangle=\left\langle u_{z} \theta\right\rangle .
$$

Applying subsequently (14), (10)-(12), (9), (1), and (13), we obtain

$$
\left\langle u_{z} \theta\right\rangle=\frac{\kappa H}{\Delta}\left\langle(\nabla \theta)^{2}\right\rangle .
$$

Combination of (8), (10), (1), and (14) yields

$$
\left\langle(\nabla \mathbf{u})^{2}\right\rangle=\frac{\kappa}{\Delta H^{3}} \operatorname{Ra}\left\langle u_{z} T\right\rangle=\frac{\kappa}{\Delta H^{3}} \operatorname{Ra}\left\langle u_{z} \theta\right\rangle .
$$


As soon as convection starts, $\mathbf{u}$ is not identically equal to zero and therefore $\left\langle(\nabla \mathbf{u})^{2}\right\rangle>0$, which means, according to Eq. (16), that the value $\left\langle u_{z} \theta\right\rangle$ becomes larger than zero. Extracting Ra from Eq. (16) and applying successively the Cauchy-Schwarz inequality and Eq. (15), one obtains the following estimate that holds true for any supercritical Rayleigh number:

$$
\begin{aligned}
\mathrm{Ra} & =\frac{\Delta H^{3}}{\kappa} \frac{\left\langle(\nabla \mathbf{u})^{2}\right\rangle}{\left\langle u_{z} \theta\right\rangle}=\frac{\Delta H^{3}}{\kappa} \frac{\left\langle(\nabla \mathbf{u})^{2}\right\rangle\left\langle u_{z} \theta\right\rangle}{\left\langle u_{z} \theta\right\rangle^{2}} \\
& \geqslant \frac{\Delta H^{3}}{\kappa} \frac{\left\langle(\nabla \mathbf{u})^{2}\right\rangle\left\langle u_{z} \theta\right\rangle}{\left\langle u_{z}^{2}\right\rangle\left\langle\theta^{2}\right\rangle}=H^{4} \frac{\left\langle(\nabla \mathbf{u})^{2}\right\rangle\left\langle(\nabla \theta)^{2}\right\rangle}{\left\langle u_{z}^{2}\right\rangle\left\langle\theta^{2}\right\rangle} \\
& \geqslant H^{4} \frac{\left\langle(\nabla \mathbf{u})^{2}\right\rangle\left\langle(\nabla \theta)^{2}\right\rangle}{\left\langle\mathbf{u}^{2}\right\rangle\left\langle\theta^{2}\right\rangle} .
\end{aligned}
$$

Formally, relation (17) holds for convective flows for any Ra. For flows close to the onset of convection, the vertical velocity component $u_{z}$ and the deviation of the temperature from the linear profile, $\theta$, are strongly correlated. Moreover, for laterally confined geometries, the viscous boundary layers near the sidewalls of the container play an important role: initial quite strong vertical motions of the fluid near and along the sidewalls, being much stronger than the horizontal motions there, determine the overall kinetic energy in the system at the Rayleigh numbers slightly larger than the critical one for the onset of convection. Therefore, relation (17) can lead to quite accurate estimates of the critical Rayleigh number for the onset of convection for laterally confined domains if one precisely estimates the greatest lower bound of the right-hand side term in (17), for all theoretically realizable convective flows in the considered domains.

One should also notice that the here developed approach can be considered as belonging to the class of variational methods of energy (see, e.g., Joseph [45] and Goluskin [46]), understood in a general sense. These methods to investigate the fluid system stability, having a long history (see, for example, Reynolds [47] and Orr [48]), are aimed to localize the regions in the control parameter space of the fluid system, where the global stability of the system is guaranteed so that the energy of any introduced velocity or temperature fluctuations unavoidably decreases as time goes on. Thus one can consider the linear stability theory and these variational methods as complementary ones, as the former theory delivers conditions (or the range of the control parameters) under which the fluid system is definitely unstable, while the latter methods give conditions under which the system is stable for sure. For some fluid systems the two sets of the critical control parameters, i.e., delivered by the linear stability theory and the variational method of energy, can be generally different and therefore the limits of these two approaches, i.e., the strict bounds on the conditions for the definite instability and definite stability might be different. However, in the case of Oberbeck-Boussinesq Rayleigh-Bénard convection, which we consider here, these limits coincide, which means that instabilities at a Rayleigh number, lower than predicted as a strict bound by the linear stability theory, do not exist (i.e., there are no arbitrary nonlinear subcritical instabilities). This was first proved by V. S. Sorokin in 1953, and then later, independently, by other authors (see $[44,49$ 52]). Thus, the critical Rayleigh number for the definite stability or instability is the same and equals $\min \frac{\Delta H^{3}}{\kappa} \frac{\left\langle(\nabla \mathbf{u})^{2}\right\rangle}{\left\langle u_{z} \theta\right\rangle}$ [cf. relation (17)], where the minimum is sought among all possible convective flow fields in the considered container, which satisfy the imposed boundary conditions. Knowing this fact, however, does not diminish the intrigue: we still need precise, but fairly easily computable estimates of the critical Rayleigh numbers for the onset of convection, for convection cells of different shapes. Assuming that $\mathbf{u}$ and $\theta$ can be any arbitrary fields, not necessarily satisfying the governing equations (3)-(5), one can relatively easy calculate the mathematically strict lower bounds for the right-hand side of (17) for ordinary domains, like circular cylinder or parallelepiped. However, to obtain realistic estimates of the critical Rayleigh number for the onset of convection, one needs to take into account all relevant properties of the functions $\mathbf{u}$ and $\theta$, which is of course not that trivial. 
In the following subsections, we will briefly discuss mathematically strict lower bounds for the critical Rayleigh number that one can derive from the relation (17) and will focus on more realistic and quite precise estimates of the critical Rayleigh numbers for the onset of convection in cylindrical and parallelepiped domains while making physically reasonable assumptions on the convective flows at the very onset of convection in confined geometries.

\section{B. Poincaré-Friedrichs inequalities and the principle dependence of the critical Rayleigh number on the domain aspect ratio}

The chain of the relations (17) can be further continued by applying inequalities of the PoincaréFriedrichs type to the velocity field $\mathbf{u}$ and to the reduced temperature $\theta$ and taking into account the corresponding boundary conditions and the specific geometry of the container. With that, we will obtain a (positive) lower bound in (17) and thus a lower bound for the Rayleigh number, below which a motion in a Rayleigh-Bénard cell cannot occur.

The Poincaré inequality

$$
\int_{\Omega} g^{2} d \Omega \leqslant c_{1} \int_{\Omega}(\nabla g)^{2} d \Omega+\widetilde{c}_{1}\left(\int_{\Omega} g d \Omega\right)^{2}
$$

and the Friedrichs inequality

$$
\int_{\Omega} g^{2} d \Omega \leqslant c_{2} \int_{\Omega}(\nabla g)^{2} d \Omega+\widetilde{c}_{2} \int_{S} g^{2} d S
$$

hold for a function $g$ (which in our case might be the velocity or deviation of the temperature from the linear profile) defined in the here considered domain $\Omega$ with the boundary $S$. [Formally, $g$ belongs to the Sobolev space $H^{1}(\Omega)$ and $\Omega$ is a domain with a Lipschitz boundary $S$; see, for example, Rektorys [53].] The constants $c_{1}$ and $\widetilde{c}_{1}$ in (18) and $c_{2}$ and $\widetilde{c}_{2}$ in (19) are non-negative and depend on the geometrical characteristics of $\Omega$ only, but not on the function $g$.

When the average over the whole domain $\Omega$ of the function $g$ equals zero, the Poincaré inequality (18) reduces to

$$
\left\langle g^{2}\right\rangle \leqslant c_{1}\left\langle(\nabla g)^{2}\right\rangle
$$

When the function $g$ vanishes at the domain boundary $S$, the Friedrichs inequality (19) takes a similar form:

$$
\left\langle g^{2}\right\rangle \leqslant c_{2}\left\langle(\nabla g)^{2}\right\rangle
$$

Although the reduced inequalities (20) and (21) look similar, the constants are generally different. For any bounded and convex domain $\Omega \subset \mathbb{R}^{3}$

$$
0<c_{2} \leqslant c_{1} \leqslant[\operatorname{diam}(\Omega) / \pi]^{2}
$$

holds true, where $\operatorname{diam}(\Omega)$ is the maximum distance between any two points of the domain $\Omega$ (i.e., the diameter of the domain $\Omega$ ); see [54]). This implies, in particular, that for any defined in a bounded and convex domain $\Omega$, square integrable function $g$, having square integrable gradient $\nabla g$, we will find to hold

$$
\frac{\left\langle(\nabla g)^{2}\right\rangle}{\left\langle g^{2}\right\rangle} \geqslant \frac{1}{c_{2}} \geqslant \frac{1}{c_{1}} \geqslant\left[\frac{\pi}{\operatorname{diam}(\Omega)}\right]^{2}>0 .
$$

Applying (23) to the reduced temperature $\theta$ and to the velocity and substituting this into (17), one obtains that the critical Rayleigh number $\mathrm{Ra}_{c, \Gamma}$ for the onset of convection satisfies

$$
\operatorname{Ra}_{c, \Gamma} \geqslant\left[\frac{\pi H}{\operatorname{diam}(\Omega)}\right]^{4}
$$


Although the estimate (24) of the critical Rayleigh number $\mathrm{Ra}_{c, \Gamma}$ for the onset of convection in any bounded convex domain $\Omega$ is not precise for the case of ordinary domains like circular cylinder or parallelepiped, it gives us a hint that geometrical characteristics of the Rayleigh-Bénard cell are important. (More accurate estimates for cylindrical and parallelepiped domains we will derive in the following subsections.)

From Eq. (22) it follows also that among all boundary conditions for the sidewalls, which guarantee that averages of $\theta$ over the whole domain vanishes and that relation (17) holds, the largest critical Rayleigh number $\operatorname{Ra}_{c, \Gamma}$ for the onset of convection will be obtained when $\theta$ vanishes at the sidewalls. Thus, one can expect larger $\mathrm{Ra}_{c, \Gamma}$ for the case of conducting sidewalls (i.e., when the temperature $T$ is linear at the sidewalls and thus $\theta$ vanishes there) compared to the case of adiabatic sidewalls.

\section{Variational characterization of the critical Rayleigh number on the domain aspect ratio}

To estimate the lower bounds of the quantities like $\left\langle(\nabla g)^{2}\right\rangle /\left\langle g^{2}\right\rangle$ in (17), we will use the variational characterization of the lowest eigenvalues of the Laplace operator $(-\Delta)$ in the domain $\Omega$,

$$
\Delta g+\lambda g=0 \text { in } \Omega,
$$

with Dirichlet boundary condition,

$$
g=0 \quad \text { on the boundary } S \text { of } \Omega,
$$

or with Neumann boundary condition,

$$
\partial g / \partial n=0 \quad \text { on the boundary } S \text { of } \Omega,
$$

or with mixed boundary conditions, where on disjoint parts of the boundary either Neumann or Dirichlet boundary conditions are satisfied. Dirichlet boundary conditions (26) are associated with the no-slip boundary conditions for the velocity at all walls and also with the boundary conditions for the reduced temperature $\theta$ at the plates and at conducting sidewalls. Neumann boundary conditions (27) are associated with the boundary conditions for $\theta$ at the adiabatic sidewalls.

The variational characterization of the lowest (positive) eigenvalue $\lambda_{1}$ of the Laplace operator is known to be

$$
\inf _{g \neq 0} \frac{\left\langle(\nabla g)^{2}\right\rangle}{\left\langle g^{2}\right\rangle}=\lambda_{1}
$$

where the infimum is sought among the functions that satisfy the corresponding boundary conditions (see, e.g., [55-57]). (The eigenvalues here have the dimension of inversed squared length.) It is obvious that for any fixed boundary conditions, the eigenvalues of the Laplace operator can depend only on the geometry of the domain in which the operator is defined. In particular, for Dirichlet, Neumann, or mixed boundary conditions, the lowest eigenvalue $\lambda_{1}$ depends on the geometrical properties of the domain $\Omega$ only.

Thus, using Eq. (28), from relation (17) we obtain that the critical Rayleigh number for the onset of convection $\mathrm{Ra}_{c, \Gamma}$ is bounded as follows:

$$
\operatorname{Ra}_{c, \Gamma} \gtrsim H^{4} \lambda_{u} \lambda_{\theta},
$$

where $\lambda_{u}$ and $\lambda_{\theta}$ are the lowest relevant eigenvalues of the Laplace operator in the considered domain $\Omega$ and for the corresponding boundary conditions for the velocity and temperature, respectively. Here we also used the fact that from

$$
\left\langle\left(\nabla u_{x}\right)^{2}\right\rangle \geqslant \lambda_{u}\left\langle u_{x}^{2}\right\rangle, \quad\left\langle\left(\nabla u_{y}\right)^{2}\right\rangle \geqslant \lambda_{u}\left\langle u_{y}^{2}\right\rangle, \quad \text { and } \quad\left\langle\left(\nabla u_{z}\right)^{2}\right\rangle \geqslant \lambda_{u}\left\langle u_{z}^{2}\right\rangle,
$$

for

$$
(\nabla \mathbf{u})^{2} \equiv\left(\nabla u_{x}\right)^{2}+\left(\nabla u_{y}\right)^{2}+\left(\nabla u_{z}\right)^{2}
$$


the following relation holds:

$$
\left\langle(\nabla \mathbf{u})^{2}\right\rangle \geqslant \lambda_{u}\left\langle\mathbf{u}^{2}\right\rangle .
$$

The properties of the Laplace operator, its eigenfunctions and eigenvalues are well investigated (see $[55,58])$. In the next subsections we will consider eigenfunctions and eigenvalues of the Laplace operator for simple geometries like parallelepiped and circular cylinder. As we will see, for these geometries and for both Dirichlet and Neumann boundary conditions, it holds that

$$
\begin{aligned}
& \lambda_{u} \propto H^{-2}\left(1+c_{u} \Gamma^{-2}\right), \\
& \lambda_{\theta} \propto H^{-2}\left(1+c_{\theta} \Gamma^{-2}\right)
\end{aligned}
$$

for certain constants $c_{u}>0$ and $c_{\theta}>0$. Combining (29) with (33) and (34), we will significantly improve the approximation (24) and will accurately estimate the dependence on the domain aspect ratio $\Gamma$ of the critical Rayleigh number for the onset of convection, in particular for small values of $\Gamma$.

\section{Estimate of the critical Rayleigh number for the onset of convection in parallelepiped Rayleigh-Bénard cells}

Let us consider the eigenvalue problem (25) for the Laplace operator $(-\Delta), \Delta=\partial_{x}^{2}+\partial_{y}^{2}+\partial_{z}^{2}$, in a domain $\Omega \equiv\left(0, D_{x}\right) \times\left(0, D_{y}\right) \times(0, H)$ with Dirichlet boundary conditions (26) or Neumann boundary conditions (27) at the boundary $S$ of the domain $\Omega$. The eigenfunctions take the form

$$
g(x, y, z)=g_{x}(x) g_{y}(y) g_{z}(z)
$$

with the eigenvalues

$$
\lambda=\lambda_{x}+\lambda_{y}+\lambda_{z},
$$

where for Dirichlet boundary conditions

$$
\begin{array}{ll}
g_{x}(x)=\sin \left(\pi n_{x} x / D_{x}\right), & \lambda_{x}=\pi^{2} n_{x}^{2} / D_{x}^{2}, \\
g_{y}(y)=\sin \left(\pi n_{y} y / D_{y}\right), & \lambda_{y}=\pi^{2} n_{y}^{2} / D_{y}^{2}, \\
g_{z}(z)=\sin \left(\pi n_{z} z / H\right), & \lambda_{z}=\pi^{2} n_{z}^{2} / H^{2},
\end{array}
$$

and for Neumann boundary conditions

$$
\begin{array}{ll}
g_{x}(x)=\cos \left(\pi n_{x} x / D_{x}\right), & \lambda_{x}=\pi^{2} n_{x}^{2} / D_{x}^{2}, \\
g_{y}(y)=\cos \left(\pi n_{y} y / D_{y}\right), & \lambda_{y}=\pi^{2} n_{y}^{2} / D_{y}^{2}, \\
g_{z}(z)=\cos \left(\pi n_{z} z / H\right), & \lambda_{z}=\pi^{2} n_{z}^{2} / H^{2},
\end{array}
$$

with integers $n_{x} \geqslant 0, n_{y} \geqslant 0$, and $n_{z}>0$, where at least one of the integers, $n_{x}$ or $n_{y}$, is positive (see illustrations for two-dimensional rectangular domains in Fig. 1).

Estimating $\lambda_{u}$ and $\lambda_{\theta}$ with the smallest positive eigenvalues $\lambda$ (36) of the Laplace operator for nonvanishing eigenmodes (35) with respective boundary conditions, one obtains

$$
\begin{aligned}
& \lambda_{u} \geqslant \frac{\pi^{2}}{H^{2}}+\frac{\pi^{2}}{D^{2}}, \\
& \lambda_{\theta} \geqslant \frac{\pi^{2}}{H^{2}}+\frac{\pi^{2}}{D^{2}} .
\end{aligned}
$$

Here we introduced

$$
D \equiv \max \left\{D_{x}, D_{y}\right\}
$$


(a)

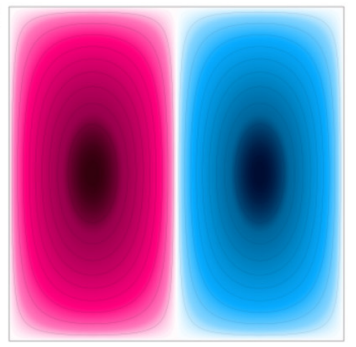

(c)

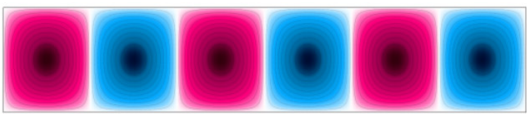

(d)

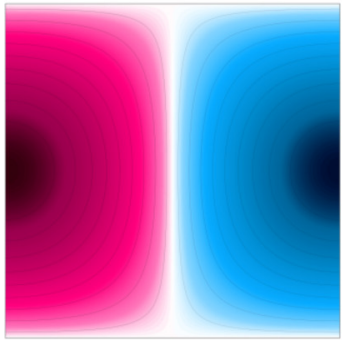

(e)

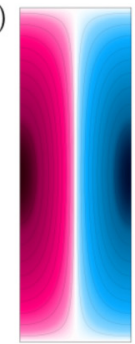

(f)

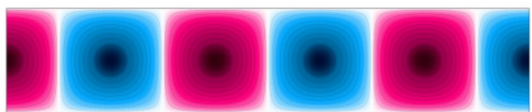

FIG. 1. Examples of eigenfunctions of the Laplace operator (possible dominating temperature modes close to the onset of convection in Rayleigh-Bénard convection) for two-dimensional domains and Dirichlet boundary conditions at the top $(z=H)$ and bottom $(z=0)$ boundaries and $(\mathrm{a}-\mathrm{c})$ Dirichlet boundary conditions at the side boundaries $(x=0$ and $x=D)$ or $(\mathrm{d}-\mathrm{f})$ Neumann boundary conditions at the side boundaries, for different aspect ratios of the domain: (a, d) $\Gamma=1$, (b, e) any case of $\Gamma<1$ (shown example is for $\Gamma=1 / 3$ ), and (c, f) $\Gamma>1$ (shown example is for $\Gamma=5$ ). All modes have a form $(\mathrm{a}-\mathrm{c}) \sim \sin \left(\pi n_{x} x / D\right) \sin \left(\pi n_{z} z / H\right)$ for Dirichlet boundary conditions at the side boundaries or $(\mathrm{d}-\mathrm{f}) \sim \cos \left(\pi n_{x} x / D\right) \sin \left(\pi n_{z} z / H\right)$ for Neumann boundary conditions at the side boundaries. Pink and blue correspond to warm or cold fluid, respectively.

so that the aspect ratio of the domain $\Omega$ is $\Gamma=D / H$. Using (43) and (44) one can derive the estimate (29), which will then hold for any smooth function $g$ that satisfies the corresponding boundary conditions and which might be thought of as the reduced temperature or the velocity components. With the relations (43) and (44) one finalizes a mathematically rigorous proof of the relations (33) and (34) which, if combined with the derived relation (29), leads to

$$
\operatorname{Ra}_{c, \Gamma} \geqslant H^{4}\left(\frac{\pi^{2}}{H^{2}}+\frac{\pi^{2}}{D^{2}}\right)^{2}=\pi^{4}\left(1+\Gamma^{-2}\right)^{2} .
$$

This is a stronger result than relation (24), as $1+\Gamma^{-2}>1 /\left(1+\Gamma^{2}\right)$. We see that the critical Rayleigh number for the onset of convection must grow as $\Gamma^{-4}$ when $\Gamma \rightarrow 0$.

While deriving the estimates (43) and (44) for a function $g$, we have not used any specific features of the velocity or temperature, which the function $g$ is sought for. To derive (46) we used relation (29), the derivation of which, in turn, is based on relation (17), which involves already some integral information about the flow, like the balance of the dissipation rates and connection to the dimensionless heat transport, i.e., the Nusselt number. However, all the so far used information is about the time- and volume-averaged quantities and the specific properties of the velocity and temperature, for instance, their spatial structures or that they satisfy the Navier-Stokes (3), energy (4), and continuity (5) equations, have not yet been used. While applying some physical arguments about the flow characteristics, we can refine the mathematically strict relation (46), to obtain more realistic estimates of the critical Rayleigh number for the onset of convection in laterally confined geometries.

Indeed, while deriving the estimates (43) and (44), we assumed only that the considered functions are smooth and satisfy the prescribed boundary conditions. A set of generally possible flow states is, however, quite restricted, and, moreover, all possible states by the onset of convection form only a subset of the set of all possible states for supercritical Rayleigh numbers. First of all, the functions that describe the flow close to the onset of convection should satisfy the continuity equation. This implies that the velocity profiles should change signs in the vertical direction and at least in one horizontal direction. With the onset of convection, also the vertical temperature profile starts to deviate from the linear profile in a symmetric way so that the reduced temperature, $\theta=T-T_{\ell}$, 
becomes negative in the lower half of the convection cell and positive in the upper half. Since near the onset of convection, the vertical component of the velocity $u_{z}$, strongly correlates with the reduced temperature $\theta$, also the horizontal profile of $\theta$ needs to change the sign. All this means that the functions, which describe the flow components at the onset of convection, cannot be of the same sign through the entire domain (they cannot be either non-negative or nonpositive through the whole height or width of the domain), thus, they must be alternating functions (i.e., they change the sign) in the vertical direction and at least in one horizontal direction. Furthermore, we assume symmetry of the flow close to the onset of convection, and therefore, the functions that describe the flow near the onset must vanish, when averaged over the whole domain.

Thus, bearing in mind only realistic flows at the onset of convection in confined geometries, which admit alternating behavior of the velocity and temperature deviations from the steady state (i.e., that the corresponding functions change the sign in horizontal as well as in the vertical directions in the domain $\Omega$ ), we propose here (without proof) that the smallest relevant eigenvalues correspond to the first alternating eigenfunctions. The correctness of this assumption has no proof; this is rather an intuitive guess supported by the reasoning from the previous paragraph. However, this assumption, being the only one in our analysis, leads to very accurate predictions of the critical Rayleigh numbers for the onset of convection in laterally confined convection cells, as we will see later. Thus, with the requirement that the leading eigenmodes are alternating functions we exclude from the consideration Dirichlet eigenmodes (37)-(39) for the values of $n_{x}, n_{y}$, and $n_{z}$ equal to one, and Neumann eigenmodes (40)-(42) for zero values of $n_{x}, n_{y}$, and $n_{z}$.

With that, taking into account that the velocity satisfies Dirichlet (no-slip) boundary conditions at all boundaries, for $\lambda_{u}$ we obtain

$$
\lambda_{u}=\frac{4 \pi^{2}}{H^{2}}+\min \left\{\frac{4 \pi^{2}}{D_{x}^{2}}, \frac{4 \pi^{2}}{D_{y}^{2}}\right\}=\frac{4 \pi^{2}}{H^{2}}+\frac{4 \pi^{2}}{D^{2}}=\frac{4 \pi^{2}}{H^{2}}\left(1+\frac{1}{\Gamma^{2}}\right) .
$$

Here and in the following we assume that $D_{y} \leqslant D_{x}=D$.

The leading eigenvalue $\lambda_{u}$ (47) for Dirichlet boundary conditions can be interpreted as an eigenvalue (36) for a quasi-two-dimensional eigenmode $g$; see Eqs. (35) and (37)-(39) with $n_{x}=2$, $n_{y}=0$, and $n_{z}=2$, which has a four-roll (two-by-two) structure. This eigenvalue corresponds to the first most energetic mode that occurs right after immediate switch on of the heating and cooling of the plates and that further develops into a well-known stable state with rolls attached to each other and aligned in a horizontal direction only, with $n_{z}=1$ and $n_{x}=2$ in the case $\Gamma \lesssim 1$ or $n_{z}=1$ and $n_{x} \geqslant 2$ in the case $\Gamma \gtrsim 1$.

The most energetic eigenmode of the developed stable state with multiple rolls attached to each other corresponds to an eigenvalue $\tilde{\lambda}_{u}$, which certainly cannot be larger than $\lambda_{u}$. The stable state with multiple rolls and the two-by-two mode can also perfectly match, so that $\tilde{\lambda}_{u}=\lambda_{u}$, through the property of the degeneracy of the Laplace eigenvalues. The phenomenon of the degeneracy of the Laplace eigenvalues for Dirichlet boundary conditions in spatially symmetrical systems is known from different problems of quantum and classical mechanics, e.g., the vibration of a rectangular membrane. Any degenerate eigenvalue corresponds to more than one linearly independent eigenmode. For example, an eigenmode $g$, [see Eq. (35) with (37)-(39)] with the mode numbers $n_{x}, n_{y}$, and $n_{z}$ and another eigenmode $\widetilde{g}$ with the mode numbers $\widetilde{n}_{x}, \widetilde{n}_{y}$, and $\widetilde{n}_{z}$ correspond to the same eigenvalue if

$$
\left(\frac{n_{x}}{D_{x}}\right)^{2}+\left(\frac{n_{y}}{D_{y}}\right)^{2}+\left(\frac{n_{z}}{H}\right)^{2}=\left(\frac{\widetilde{n}_{x}}{D_{x}}\right)^{2}+\left(\frac{\widetilde{n}_{y}}{D_{y}}\right)^{2}+\left(\frac{\widetilde{n}_{z}}{H}\right)^{2}
$$

From Eq. (48) one can see that the degeneracy of Dirichlet eigenvalues in confined rectangular domains can happen for the domain aspect ratios $D_{x} / H$ and $D_{y} / H$, which can be represented in a form of the square roots of some rational numbers. Furthermore, the eigenvalue for the case 
$n_{x}=2, n_{y}=0$ and $n_{z}=2$ will asymptotically match the eigenvalue for $\widetilde{n}_{y}=0$ and $\widetilde{n}_{z}=1$ if $\tilde{n}_{x} \approx$ $\sqrt{4+3 \Gamma^{2}}$. This resembles the cases

$$
\begin{aligned}
& \tilde{n}_{x} \rightarrow 2 \text { for } \Gamma \rightarrow 0, \\
& \tilde{n}_{x} \sim \Gamma \text { for } \Gamma \rightarrow \infty,
\end{aligned}
$$

of stable states by slightly supercritical convection.

When the reduced temperature also satisfies Dirichlet boundary conditions (i.e., the sidewalls are conducting and the plates are isothermal),

$$
\lambda_{\theta}=\lambda_{u}
$$

When the boundary conditions for the temperature are Dirichlet ones at the plates and Neumann (adiabatic) at the sidewalls, from relations (36) and (39) for $n_{z}=2$, (40) for $n_{x}=1$ and (41) for $n_{y}=1$ we obtain

$$
\lambda_{\theta}=\frac{4 \pi^{2}}{H^{2}}+\frac{\pi^{2}}{D_{x}^{2}}+\frac{\pi^{2}}{D_{y}^{2}}=\frac{4 \pi^{2}}{H^{2}}\left(1+\frac{1}{4 \Gamma_{x}^{2}}+\frac{1}{4 \Gamma_{y}^{2}}\right)
$$

where

$$
\Gamma_{x} \equiv D_{x} / H, \quad \Gamma_{y} \equiv D_{y} / H .
$$

Now we can estimate the critical Rayleigh number $\mathrm{Ra}_{c, \Gamma}$ as a function of the domain aspect ratio $\Gamma$, for parallelepiped domains with no-slip boundary conditions for the velocity at all boundaries. From (29), (47), and (51), we obtain $\mathrm{Ra}_{c, \Gamma}$ for the conducting sidewalls:

$$
\operatorname{Ra}_{c, \Gamma} \approx(2 \pi)^{4}\left(1+\frac{1}{\Gamma^{2}}\right)^{2} .
$$

For adiabatic sidewalls, from (29), (47), and (52) we obtain

$$
\begin{array}{ll}
\operatorname{Ra}_{c, \Gamma} \approx(2 \pi)^{4}\left(1+\frac{1}{\Gamma^{2}}\right)\left(1+\frac{1}{4 \Gamma^{2}}\right) & \text { in 2D }, \\
\operatorname{Ra}_{c, \Gamma} \approx(2 \pi)^{4}\left(1+\frac{1}{\Gamma_{x}^{2}}\right)\left(1+\frac{1}{4 \Gamma_{x}^{2}}+\frac{1}{4 \Gamma_{y}^{2}}\right) & \text { in 3D. }
\end{array}
$$

Thus using the facts that the velocity $\mathbf{u}$ and the deviation of the temperature from the linear profile, $\theta$, satisfy the prescribed boundary conditions and relation (17), without using any other properties of $\mathbf{u}$ and $\theta$, we have derived a rigorous estimate (46) for the critical Rayleigh number for the onset of convection, $\mathrm{Ra}_{c, \Gamma}$. However, under the assumption, which is in line with the physical reasoning discussed above, that the first relevant eigenmodes in the expansions of $\mathbf{u}$ and $\theta$ in terms of the Laplace eigenfunctions are alternating (i.e., changing the sign) functions in both horizontal and vertical directions, we have derived physically more realistic estimates of $\mathrm{Ra}_{c}, \Gamma$ for laterally confined domains. For adiabatic sidewalls these estimates are Eq. (55) for two-dimensional rectangular domains and Eq. (56) for three-dimensional parallelepiped domains. When the thermal boundary conditions at the sidewalls are conducting or periodic, the critical Rayleigh number for the onset of convection can be estimated with Eq. (54).

\section{E. Estimate of the critical Rayleigh number for the onset of convection in cylindrical Rayleigh-Bénard cells}

Let us now conduct a similar analysis as in the previous section, but for cylindrical domains. This will allow us to derive the critical Rayleigh number $\mathrm{Ra}_{c}, \Gamma$ for the onset of convection in fluid layers confined in cylindrical containers. 
(a)

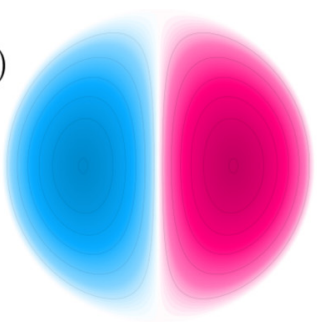

(d)

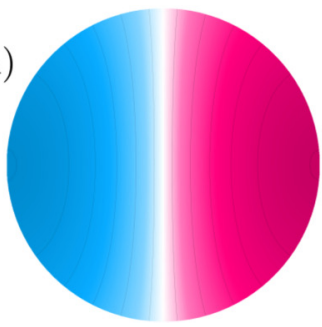

(b)

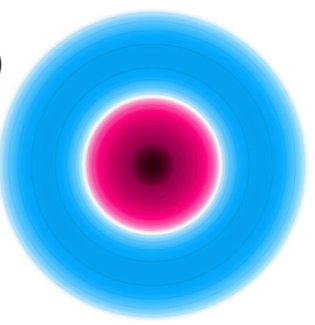

(e)

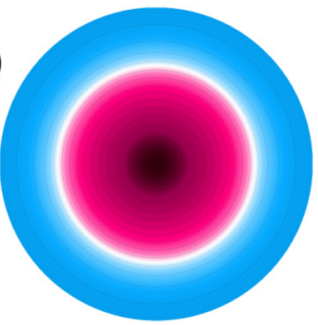

(c)

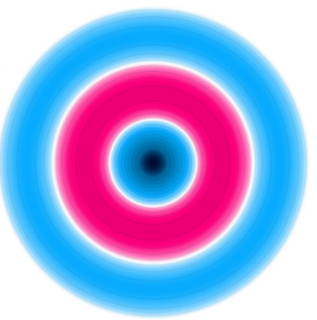

(f)

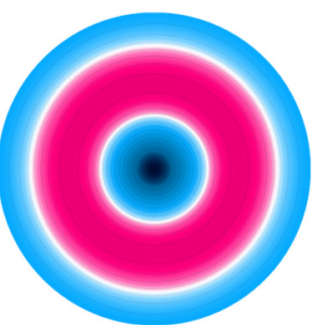

FIG. 2. Examples of eigenfunctions of the Laplace operator in a circle for $(a-c)$ Dirichlet boundary conditions at the side boundary $(r=D / 2)$ or (d-f) Neumann boundary conditions at $r=D / 2$. All shown modes have a form $\sim J_{n}\left(2 \alpha_{n k}(r / D)\right) \cos (n \phi)$, where $\alpha_{n k}$ is (a-c) the $k$ th positive root $j_{n k}$ of the Bessel function $J_{n}$, for Dirichlet boundary conditions at at $r=D / 2$, or (d-f) the $k$ th positive root $\widetilde{j}_{n k}$ of the derivative $J_{n}^{\prime}$ of the Bessel function $J_{n}$, for Neumann boundary conditions at at $r=D / 2$. These can be interpreted as possible dominating temperature modes in the central horizontal cross section of a cylindrical Rayleigh-Bénard cell, close to the onset of convection, for Dirichlet boundary conditions at the top $(z=H)$ and bottom $(z=0)$ plates, where the reduced temperature $\theta$ vanishes, and (a-c) Dirichlet (conducting) boundary conditions at the sidewall $(r=D / 2)$ or (d-f) Neumann (adiabatic) boundary conditions at the sidewall $(r=D / 2)$, for different aspect ratios of the container: $(\mathrm{a}, \mathrm{d}) \Gamma \leqslant 1,(\mathrm{~b}, \mathrm{e}) \Gamma \approx 2$, and $(\mathrm{c}, \mathrm{f}) \Gamma \approx 5$. Color scale as in Fig. 1.

We consider the eigenvalue problem (25) for the Laplace operator $(-\Delta)[55,58]$,

$$
\Delta=\partial_{r}^{2}+(1 / r) \partial_{r}+\left(1 / r^{2}\right) \partial_{\phi}^{2}+\partial_{z}^{2},
$$

in a cylindrical domain $\Omega$ with the diameter $D$ and height $H$, with Dirichlet boundary conditions (26) or Neumann boundary conditions (27) at the boundary $S$ of the domain $\Omega$. The eigenfunctions take the form

$$
f(r, \phi, z)=J_{n}\left(2 \alpha_{n k} r / D\right) \cos (n \phi) g_{z}(z),
$$

where $g_{z}$ is given by Eq. (39), $J_{n}$ is the Bessel function of the first kind and the coefficients $\alpha_{n k}$ are determined by the boundary condition at the sidewall (see illustrations for two-dimensional circular domains in Fig. 2). Other eigenvalues are similar to those from Eq. (58), but with $\sin (n \phi)(n \neq 0)$ instead of $\cos (n \phi)$, thus leading to the same eigenvalues. As their consideration would not change the results (the leading eigenvalues) and therefore the principle conclusions on the critical Rayleigh number $\mathrm{Ra}_{c, \Gamma}$, we do not discuss them here.

The eigenvalues that correspond to the eigenmodes (58) are

$$
\lambda=\lambda_{\phi, r}+\lambda_{z}
$$

with

$$
\lambda_{\phi, r} \equiv 4 \alpha_{n k}^{2} / D^{2}
$$

and $\lambda_{z}$ given in (39). For Dirichlet boundary conditions at the sidewall of the cylindrical container, the values of $\alpha_{n k}$ are the positive roots $j_{n k}$ of the Bessel function $J_{n}$. For Neumann boundary 
conditions, $\alpha_{n k}$ are the positive roots of the derivative of the Bessel function, i.e., of $J_{n}^{\prime}$; these roots are denoted as $\widetilde{j}_{n k}$.

With similar argumentation as in the previous section for parallelepiped containers, also for cylindrical domains, we propose that the smallest relevant eigenvalues correspond to the eigenfunctions that change sign in the vertical direction and at least in one horizontal direction $(r$ or $\phi)$ of the domain $\Omega$, i.e., the leading eigenfunctions are alternating functions. This assumption (or proposition) is in line with the symmetry, continuity, and vanishing in mean flow at the onset of convection and is, as already mentioned, the only one assumption made in our analysis, which, however, leads to precise estimates of the critical Rayleigh numbers $\operatorname{Ra}_{c, \Gamma}$ for the onset of convection in confined cylindrical containers of the aspect ratio $\Gamma$, as we will show later.

Following our proposition, we consider $J_{n}\left(2 \alpha_{n k} r / D\right) \cos (n \phi)$ in Eq. (58) for only those combinations of $n$ and $k$ that make eigenfunctions $f(r, \phi, z)$ alternating in the vertical direction $z$ as well in the horizontal plane $\phi-r$, i.e., only those $J_{n}\left(2 \alpha_{n k} r / D\right) \cos (n \phi)$ that change sign in a $\phi-r$ plane, at any height $z$. For Dirichlet boundary conditions, this excludes from the consideration a potential choice of the $\alpha_{n k}$ for $n=0$ and $k=1$ that corresponds to $\alpha_{01}=j_{01} \approx 2.4048$. Among other closest values, which are $\alpha_{02}=j_{02}=5.5201$ for $n=0$ and $k=2$ and $\alpha_{11}=j_{11}=3.8317$ for $n=1$ and $k=1$, the value of $j_{11}$ is smaller and therefore is taken into the estimate. Thus the smallest relevant eigenvalues correspond to $J_{1}(n=1)$ with $k=1$, where

$$
\alpha_{11}=j_{11} \approx 3.8317
$$

for Dirichlet boundary conditions at the sidewall, and

$$
\alpha_{11}=\widetilde{j}_{11} \approx 1.8412
$$

for Neumann boundary conditions.

The smallest relevant $\lambda_{z}$, Eq. (39), which is used in (59) corresponds to $n_{z}=2$. With this and Eqs. (39), (60), and (59) we obtain

$$
\lambda=\frac{4 \pi^{2}}{H^{2}}+\frac{4 \alpha_{11}^{2}}{D^{2}}
$$

Taking into account that the velocity satisfies Dirichlet (no-slip) boundary conditions at all boundaries, from (63) and (61) for $\lambda_{u}$ we obtain

$$
\lambda_{u}=\frac{4 \pi^{2}}{H^{2}}+\frac{4 j_{11}^{2}}{D^{2}}=\frac{4 \pi^{2}}{H^{2}}\left(1+\frac{j_{11}^{2}}{\pi^{2} \Gamma^{2}}\right) \approx \frac{4 \pi^{2}}{H^{2}}\left(1+\frac{1.4876}{\Gamma^{2}}\right) .
$$

When the sidewall is conducting and the plates are isothermal, i.e., when the reduced temperature also satisfies Dirichlet boundary conditions, we have

$$
\lambda_{\theta}=\lambda_{u} .
$$

In the case when the boundary conditions for the temperature are Dirichlet ones at the plates and Neumann (adiabatic) at the sidewall, from relations (63) and (62) we obtain

$$
\lambda_{\theta}=\frac{4 \pi^{2}}{H^{2}}+\frac{4\left(\widetilde{j}_{11}\right)^{2}}{D^{2}}=\frac{4 \pi^{2}}{H^{2}}\left(1+\frac{\left(\widetilde{j}_{11}\right)^{2}}{\pi^{2} \Gamma^{2}}\right) \approx \frac{4 \pi^{2}}{H^{2}}\left(1+\frac{0.3435}{\Gamma^{2}}\right) .
$$

Now we can estimate the critical Rayleigh number $\mathrm{Ra}_{c, \Gamma}$ for the onset of convection as a function of the aspect ratio $\Gamma=D / H$ of the cylindrical container with no-slip boundary conditions for the velocity at all boundaries. From (29), (64), and (65), we obtain $\mathrm{Ra}_{c, \Gamma}$ for the case of the conducting sidewall:

$$
\operatorname{Ra}_{c, \Gamma} \approx(2 \pi)^{4}\left(1+\frac{j_{11}^{2}}{\pi^{2} \Gamma^{2}}\right)^{2} \approx(2 \pi)^{4}\left(1+\frac{1.4876}{\Gamma^{2}}\right)^{2}
$$


(a)

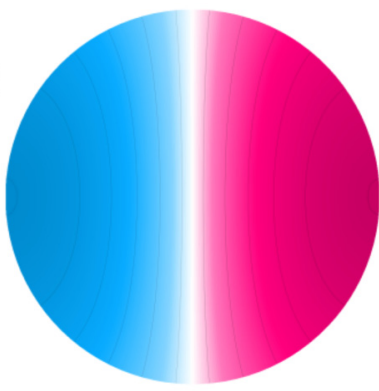

(c)

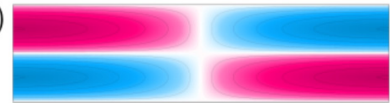

(b)

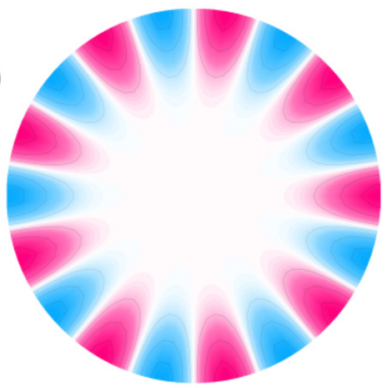

(d)

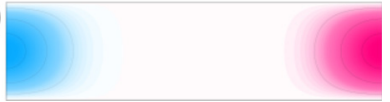

FIG. 3. Example of a pair of eigenfunctions, $(a, c)$ " $2 \times 2$ mode" and (b, d) "wall mode," that correspond to the same (degenerate) eigenvalue of the Laplace operator (possible dominating temperature modes close to the onset of Rayleigh-Bénard convection) for a cylindrical domain with the aspect ratio $\Gamma \approx 3.878$ and Dirichlet boundary conditions at the top $(z=H)$ and bottom $(z=0)$ boundaries and Neumann boundary conditions at the side boundary, which are plotted in the horizontal cross sections (a, b), for (a) $z / H=1 / 4$ and (b) $z / H=1 / 2$, and in the central vertical cross sections (c, d). Both eigenfunctions, in (a, c) and in (b, d), have a form $J_{n}\left(2 \widetilde{j}_{n k} r / D\right) \cos (n \phi) \sin \left(\pi n_{z} z / H\right)$ and correspond to the same eigenvalue $\lambda=4 \widetilde{j}_{n k}^{2} / D^{2}+\pi^{2} n_{z}^{2} / H^{2}$, where (a, c) $n=1, k=1, n_{z}=2$ and $\lambda \approx 4 \pi^{2} H^{-2}\left(1+0.34 \Gamma^{-2}\right)$ and (b, d) $n=9, k=1, n_{z}=1$ and $\lambda \approx 4 \pi^{2} H^{-2}\left(0.25+11.63 \Gamma^{-2}\right)$, both for $\Gamma \approx 3.878$. Here $J_{n}$ is the Bessel function $J_{n}$ and $\widetilde{j}_{n k}$ is the $k$ th root of the derivative of the Bessel function $J_{n}$. Pink and blue correspond to warm or cold fluid, respectively.

For the adiabatic sidewall, from (29), (64), and (66) we obtain

$$
\operatorname{Ra}_{c, \Gamma} \approx(2 \pi)^{4}\left(1+\frac{j_{11}^{2}}{\pi^{2} \Gamma^{2}}\right)\left(1+\frac{\left(\tilde{j}_{11}\right)^{2}}{\pi^{2} \Gamma^{2}}\right) \approx(2 \pi)^{4}\left(1+\frac{1.4876}{\Gamma^{2}}\right)\left(1+\frac{0.3435}{\Gamma^{2}}\right) .
$$

Thus from the relation (17), under the assumption that in a laterally confined cylinder, in the expansions of the velocity $\mathbf{u}$ and the deviation of the temperature from the steady-state profile, $\theta$, in terms of the Laplace eigenfunctions, the first relevant eigenmodes change sign (alternate) in both horizontal and vertical directions, we have derived estimates of the critical Rayleigh number for the onset of convection, $\mathrm{Ra}_{c, \Gamma}$; for the case of conducting sidewall, see Eqs. (67), and for the case of adiabatic sidewall, see Eqs. (68).

We should mention that degenerate eigenvalues also appear in cylindrical domains. Two illustrations are presented in Fig. 3 and Fig. 4, where we can see that the "two-by-two" mode with $n=1, k=1$, and $n_{z}=2$ [see Figs. 3(a) and 3(b) and Figs. 4(a) and 4(b)], which, in the above analysis, was considered as the first relevant mode, can have exactly the same eigenvalue as a "wall mode" [Figs. 3(b) and 3(d)] or as a "donut mode" [Figs. 4(b) and 4(d)] if the aspect ratio of the domain allows this. Thus, the mode with $n=1, k=1$ and $n_{z}=2$ can transform into another, a quite different in shape but energetically similar mode that corresponds to the same or smaller eigenvalue. Whether the leading eigenvalue is degenerate or not depends on the aspect ratio of the cylinder: only large values of $\Gamma>1$ admit degenerate eigenvalues with that for $n=1, k=1$ and $n_{z}=2$.

The "two-by-two mode" with $n=1, k=1$ and $n_{z}=2$ can be associated with an initial flow motion in laterally confined containers, where the instability so to say starts from the corners of the container, in a quite symmetric way. If the aspect ratio $\Gamma$ is small, the mode with $n=1, k=1$ should remain to be the most energetic because there are no other realisable eigenmodes that correspond to the same or smaller eigenvalue. However, when the aspect ratio $\Gamma$ is large, there appear other mode options due to the above-discussed degeneracy of the eigenvalues. Thus for large aspect ratio containers one might observe "donut modes" as in Figs. 4(b) and 4(d) or even "wall modes" like 
(a)

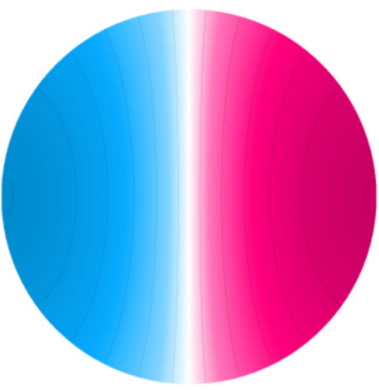

(c)

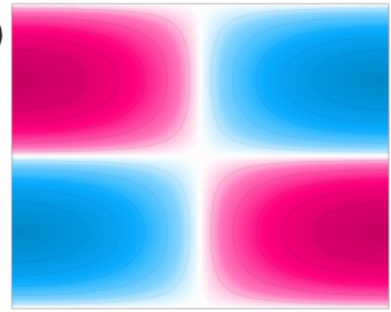

(b)

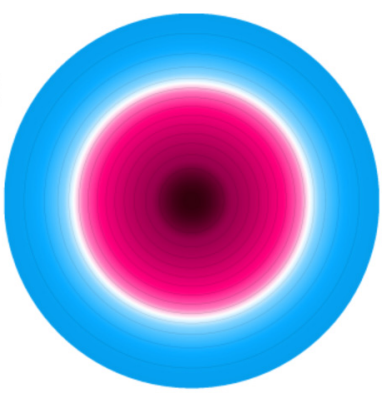

(d)

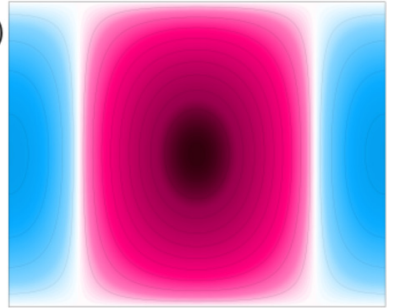

FIG. 4. Another example of a pair of eigenfunctions, (a, c) " $2 \times 2$ mode" and (b, d) "donut mode," that correspond to the same (degenerate) eigenvalue of the Laplace operator (possible dominating temperature modes close to the onset of Rayleigh-Bénard convection) for a cylindrical domain with the aspect ratio $\Gamma \approx$ 1.235 and Dirichlet boundary conditions at the top $(z=H)$ and bottom $(z=0)$ boundaries and Neumann boundary conditions at the side boundary, which are plotted in the horizontal cross sections (a, b), for (a) $z / H=$ $1 / 4$ and (b) $z / H=1 / 2$, and in the central vertical cross sections (c, d). Both eigenfunctions, in (a, c) and in (b, d), have a form $J_{n}\left(2 \widetilde{j}_{n k} r / D\right) \cos (n \phi) \sin \left(\pi n_{z} z / H\right)$ and correspond to the same eigenvalue $\lambda=4 \widetilde{j}_{n k}^{2} / D^{2}+$ $\pi^{2} n_{z}^{2} / H^{2}$, where (a, c) $n=1, k=1, n_{z}=2$, and $\lambda \approx 4 \pi^{2} H^{-2}\left(1+0.34 \Gamma^{-2}\right)$ and (b, d) $n=0, k=1, n_{z}=1$, and $\lambda \approx 4 \pi^{2} H^{-2}\left(0.25+1.488 \Gamma^{-2}\right)$, for $\Gamma \approx 1.235$. Here $J_{n}$ is the Bessel function $J_{n}$ and $\widetilde{j}_{n k}$ is the $k$ th root of the derivative of the Bessel function $J_{n}$. Pink and blue correspond to warm or cold fluid, respectively.

in Figs. 3(b) and 3(d). The preference in realization and dominance of this or that mode depend on specific values of the container aspect ratio $\Gamma$.

\section{F. Illustration from the classical linear stability analysis for the Rayleigh's free-slip case}

Below we illustrate why the consideration of the flow near the onset of convection as expansion in terms of the Laplace eigenfunctions can be useful and briefly discuss why the agreement of our analysis with the classical linear stability analysis is good, though the underlying ansatz is different.

Let us consider Rayleigh's example [5] of the onset of convection in an infinite fluid layer for free-slip boundary conditions at the plates. Following the fundamental idea of the linear stability analysis [24,25], we can rewrite the governing equations (3)-(4) in terms of the velocity $\mathbf{u}$ and reduced temperature $\theta$, using the decomposition (13), in Cartesian coordinates $(x, y, z)$. According to the concept of the linear stability analysis, at the onset of motion, the contribution from the nonlinear terms is negligible, therefore one obtains

$$
\begin{gathered}
\partial_{t} \mathbf{u}+\nabla p=v \nabla^{2} \mathbf{u}+\alpha g \theta \mathbf{e}_{z}, \\
\partial_{t} \theta=\kappa \nabla^{2} \theta+u_{z} \Delta / H .
\end{gathered}
$$

Using $\Delta, H$ and $\kappa / H$ as the reference temperature, distance, and velocity, one makes these equations dimensionless. (Below in this section we use the same notations for the dimensionless quantities as 
for the corresponding dimensional quantities above.) Applying twice the curl operator $[\nabla \times(\nabla \times)]$ to Eq. (69) one eliminates the pressure term,

$$
\begin{gathered}
\partial_{t} \nabla^{2} u_{z}=\operatorname{Pr} \nabla^{2} \nabla^{2} u_{z}+\operatorname{Pr} \operatorname{Ra}\left(\partial_{x}^{2}+\partial_{y}^{2}\right) \theta, \\
\partial_{t} \theta=\nabla^{2} \theta+u_{z}
\end{gathered}
$$

and thus obtains

$$
\left(\partial_{t}-\nabla^{2}\right)\left(\operatorname{Pr}^{-1} \partial_{t}-\nabla^{2}\right) \nabla^{2} u_{z}=\operatorname{Ra}\left(\partial_{x}^{2}+\partial_{y}^{2}\right) u_{z} .
$$

The boundary conditions at the plate are $u_{z}=0$ and in the free-slip case also $\partial_{z}^{2 k} u_{z}=0$ for $k=$ $1,2, \ldots$, that follows from the continuity.

Let us consider $u_{z}$ in a form

$$
u_{z}=\exp (\sigma t) g(x, y, z)
$$

i.e., as a product of the amplification of the velocity with time by growth rate $\sigma$ and any eigenfunction $g(x, y, z)$ of the Laplace operator $(-\Delta), \Delta=\partial_{x}^{2}+\partial_{y}^{2}+\partial_{z}^{2}$,

$$
\begin{gathered}
g(x, y, z) \equiv g_{x, y}(x, y) g_{z}(z), \\
g_{z}(z) \equiv \sin \left(\pi n_{z} z\right), \\
\left(\partial_{x}^{2}+\partial_{y}^{2}\right) g_{x, y}+\lambda_{x, y} g_{x, y}=0, \\
\partial_{z}^{2} g_{z}+\lambda_{z} g_{z}=0,
\end{gathered}
$$

with integer $n_{z}$ and positive values of $\lambda_{x, y}$ and

$$
\lambda_{z}=\left(\pi n_{z}\right)^{2} .
$$

The function $g_{z}(z)$ (76) guarantees that the boundary conditions at the plate $(z=0)$ are met, i.e., $\partial^{2 k} u_{z}=0$ for $k=0,1,2, \ldots$ Substituting Eqs. (74)-(79) into (73), for the neutral (marginal) state $\sigma=0$ we obtain

$$
\left(\lambda_{x, y}+\lambda_{z}\right)^{3}=\lambda_{x, y} \text { Ra. }
$$

Let us recollect that in an infinite fluid layer, $\lambda_{x, y}$ can take any positive value. Therefore the onset of convection starts at $\mathrm{Ra}_{c}$, which corresponds to the minimum over all positive $\lambda_{x, y}$ values of $\mathrm{Ra}=\left(\lambda_{x, y}+\lambda_{z}\right)^{3} / \lambda_{x, y}$ that is achieved at

$$
\lambda_{x, y}=\lambda_{z} / 2
$$

The smallest $\lambda_{z}$ corresponds to $n_{z}=1$, i.e., $\lambda_{z}=\pi^{2}$, which together with Eq. (81) leads to Rayleigh's result [5]:

$$
\operatorname{Ra}_{c}=(27 / 4) \pi^{4} .
$$

Note that in the derivations that we have conducted right now, we neither assumed any specific shape of the domain nor any specific form of the function $g_{x, y}(x, y)$ in the Laplace eigenfunction $g(x, y, z)$, Eq. (75).

However, when the flow is confined inside a parallelepiped $\left[0, \Gamma_{x}\right] \times\left[0, \Gamma_{y}\right] \times[0,1]$, the function $g_{x, y}(x, y)$ must have a form

$$
g_{x, y}(x, y) \sim \sin \left(\frac{\pi n_{x} x}{\Gamma_{x}}\right) \sin \left(\frac{\pi n_{y} y}{\Gamma_{y}}\right),
$$


which leads to

$$
\lambda_{x, y}=\left(\frac{\pi n_{x}}{\Gamma_{x}}\right)^{2}+\left(\frac{\pi n_{y}}{\Gamma_{y}}\right)^{2} .
$$

In the case $\Gamma \rightarrow 0$, one cannot simply take $\lambda_{x, y}$ according to Eq. (81), because $n_{x}$ and $n_{y}$ must be integers. The closest to $\lambda_{z} / 2$ for $n_{z}=1$ value of $\lambda_{x, y}$ is estimated as

$$
\lambda_{x, y} \geqslant \frac{\pi^{2}}{\max \left\{\Gamma_{x}^{2}, \Gamma_{y}^{2}\right\}}=\frac{\pi^{2}}{\Gamma^{2}} .
$$

From this, Eq. (79) for $n_{z}=1$ and Eq. (80) we then obtain that

$$
\mathrm{Ra}_{c} \geqslant \frac{\left(\pi^{2} \Gamma^{-2}+\pi^{2}\right)^{3}}{\pi^{2} \Gamma^{-2}}>\pi^{4} \Gamma^{-4}, \quad \Gamma \rightarrow 0,
$$

which confirms again that the critical Rayleigh number grows as $\sim \Gamma^{-4}$ for tall containers, $\Gamma \rightarrow 0$. However, to form at least one roll in horizontal direction, either $n_{x}$ or $n_{y}$ must be larger than 1 . With that we obtain

$$
\operatorname{Ra}_{c} \geqslant \frac{\left[(2 \pi)^{2} \Gamma^{-2}+\pi^{2}\right]^{3}}{(2 \pi)^{2} \Gamma^{-2}} \rightarrow(2 \pi)^{4} \Gamma^{-4}, \quad \Gamma \rightarrow 0 .
$$

The analytical derivations in the classical linear stability analysis for the case of an infinite fluid layer with the no-slip boundary conditions at the plates are known to be not that easy as for the case of the free-slip plates, due to the difficulty to meet the boundary conditions at the plates. The boundary conditions for the no-slip horizontal boundaries of the fluid layer include $u_{z}=0$ as well as $\partial_{z} u_{z}=0$, which occur from the continuity equation at the plates. Therefore, it is not sufficient to consider a single mode in a form (76) in the vertical direction. Consideration of a finite number of such modes is not sufficient either. All this, as we do know, significantly complicates the analysis in the case of the no-slip boundary conditions at the plates.

When the domain is laterally confined and the boundary conditions for the velocity and temperature at the sidewalls are different (as, for example, for the no-slip conditions for the velocity and adiabatic ones for the temperature), the analysis becomes even more complicated and analytically unfeasible. Thus, in the case of different boundary conditions for the velocity and temperature at the sidewalls, it is also not sufficient to consider a single mode in the horizontal directions [i.e., in a form $\left.\sim \exp \left(i k_{x} x+i k_{y} y+\sigma t\right)\right]$, for both the velocity and the temperature, as is usually done in the classical linear stability analysis [23-25] for laterally unbounded domains.

\section{G. Comparison with the amplitude equation approximation}

Here we compare our estimates with the results obtained from the amplitude equation by Ahlers et al. [60]; see also [61-64]. The equation describes slow variations in space and time of the velocity and temperature close to the onset of convection in terms of the projection of these variables on the slowest unstable mode in the system. It allows estimating the increase in critical Rayleigh number for the onset of convection due to the lateral boundaries, for containers with $\Gamma \gtrsim 1$. In particular, under the assumption that the first unstable modes in wide cylindrical containers with adiabatic sidewalls are concentric rolls, one obtains [60]

$$
\frac{\mathrm{Ra}_{c, \Gamma}-\mathrm{Ra}_{c, \infty}}{\mathrm{Ra}_{c, \infty}} \approx \frac{1.18 \pi^{2} \xi_{0}^{2}}{\Gamma^{2}} \approx \frac{1.73}{\Gamma^{2}}, \quad \Gamma \rightarrow \infty,
$$

with $\xi_{0}^{2} \approx 0.148[60,64]$.

Our estimate (68) can be rewritten in a similar form that gives

$$
\frac{\operatorname{Ra}_{c, \Gamma}-\operatorname{Ra}_{c, \Gamma \rightarrow \infty}}{\operatorname{Ra}_{c, \Gamma \rightarrow \infty}} \approx\left(1+\frac{j_{11}^{2}}{\pi^{2} \Gamma^{2}}\right)\left(1+\frac{\left(\tilde{j}_{11}\right)^{2}}{\pi^{2} \Gamma^{2}}\right)-1 \approx \frac{1.83}{\Gamma^{2}}, \quad \Gamma \rightarrow \infty
$$


(a)

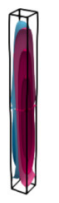

(h)

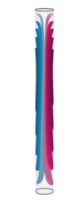

$\Gamma=1 / 10$ (b)

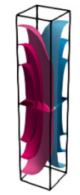

(i)

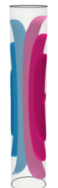

$=1 / 5$ (c)

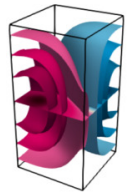

(j)

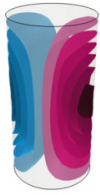

$\Gamma=1 / 2$

(d)
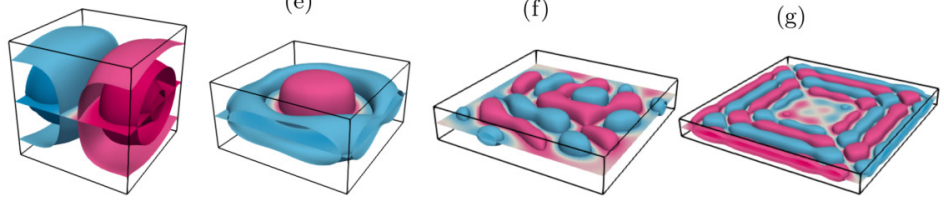

$(\mathrm{k})$

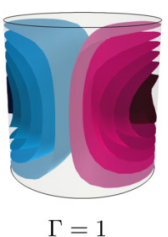

(1)

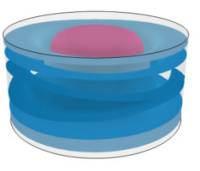

$\Gamma=2$

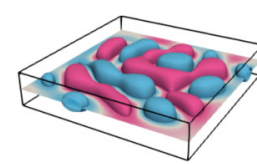

(f)

\begin{abstract}
J
\end{abstract}
(m)

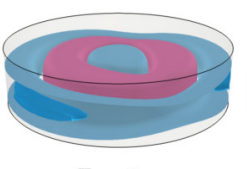

$\Gamma=5$

(g)

FIG. 5. Contours of the temperature fields in Rayleigh-Bénard convection close to the onset of convection, in parallelepiped containers with $\Gamma_{x}=\Gamma_{y}$ (upper row) and cylindrical containers (lower row) with adiabatic sidewalls, for different aspect ratios $\Gamma$ : $(\mathrm{a}, \mathrm{h}) \Gamma=1 / 10,(\mathrm{~b}, \mathrm{i}) \Gamma=1 / 5,(\mathrm{c}, \mathrm{j}) \Gamma=1 / 2,(\mathrm{~d}, \mathrm{k}) \Gamma=1,(\mathrm{e}, \mathrm{l})$ $\Gamma=2$, $(\mathrm{f}, \mathrm{m}) \Gamma=5$, and $(\mathrm{g}, \mathrm{n}) \Gamma=10$, as obtained numerically from the linearized governing equations. The color scale ranges from blue (cold fluid) to pink (warm fluid). Simulations conducted by P. Reiter; adopted from [59]. The flow structures in the horizontal direction are similar to the theoretical ones; cf. (h-k) with Fig. 2(d) for $\Gamma \leqslant 1$, (l) with Fig. $2(\mathrm{e})$ for $\Gamma \approx 2$, and $(\mathrm{m})$ with Fig. 2 (f) for $\Gamma \approx 5$.

From the comparison of the approximations (87) and (88), we conclude that the estimate from the amplitude equations [60] and our result, Eq. (68), are in good agreement for large aspect ratios of the containers, $\Gamma \gg 1$. In the other limit, for small $\Gamma$, the deviation between these two approaches is certainly huge, "because the amplitude equation is based upon the assumption of slow spatial variations and therefore strictly applicable only for large L"; see Behringer and Ahlers [64] (page 237, where $L \equiv \Gamma / 2$ ).

\section{H. Comparison with the numerical linear stability analysis}

Let us now compare the derived estimates for the onset of convection with the results from Ahlers et al. [59] for the numerically conducted linear stability analysis; see Fig. 5. The critical Rayleigh numbers $\operatorname{Ra}_{c, \Gamma}$ for the onset of convection, as functions of the domain aspect ratio $\Gamma$, are presented in Figs. 6(a) and 6(b) for two-dimensional rectangular domains, and in Figs. 6(c) and 6(d) for three-dimensional cylindrical domains, with conducting sidewalls [Figs. 6(a) and 6(c)] and adiabatic sidewalls [Figs. 6(b) and 6(d)]. As everywhere in our analysis, the boundary conditions for the velocity are no-slip.

Being quite expensive, the classical linear stability analysis in terms of the normal modes was conducted in [59] for two-dimensional domains only [see continuous blue curves in Figs. 6(a) and 6(b)]. A cheaper way to find the neutral stability curves for all studied geometries and boundary conditions was undertaken in [59], using simulations of the linearized governing equations initiated with random disturbances of small amplitudes. As soon as the kinetic energy $E_{\text {kin }}$ had reached a stable linear temporal growth (or decay), the growth rate was evaluated according to

$$
2 \sigma=d \log \left(E_{\mathrm{kin}}\right) / d t
$$

(see supplementary materials in [59]). These linear growth rates are shown with colored vertically elongated boxes in Fig. 6. The best fits of the neutral stability curves, which in [59] were sought in the form $\operatorname{Ra}_{c, \Gamma} \approx 1708\left(1+C / \Gamma^{2}\right)^{2}$, are shown in Fig. 6 with black dashed lines. The theoretical 
(a)

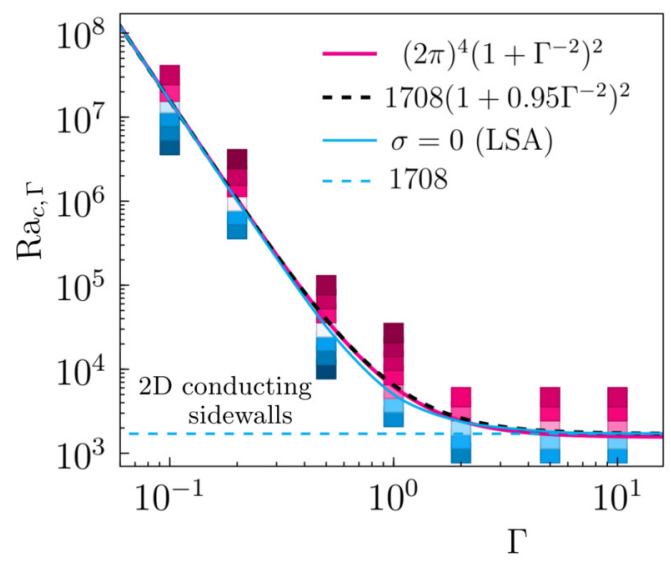

(c)

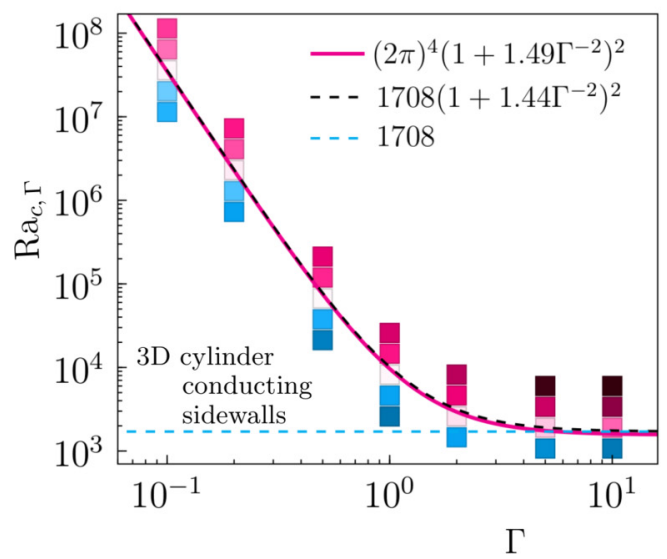

(b)

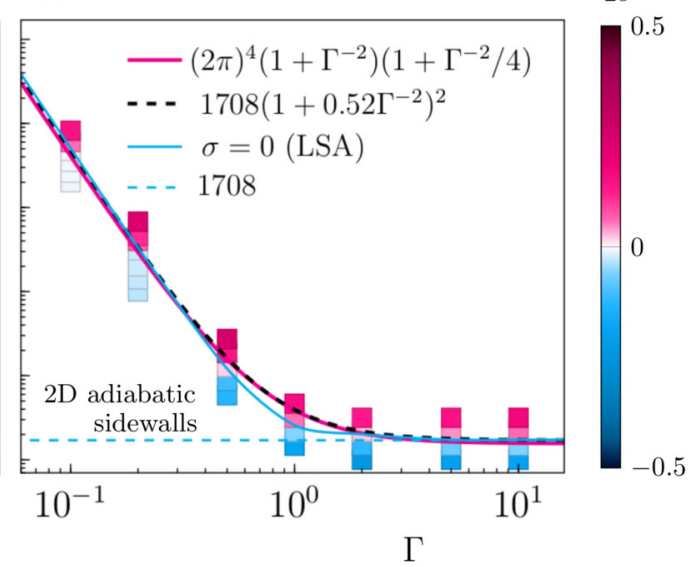

(d)

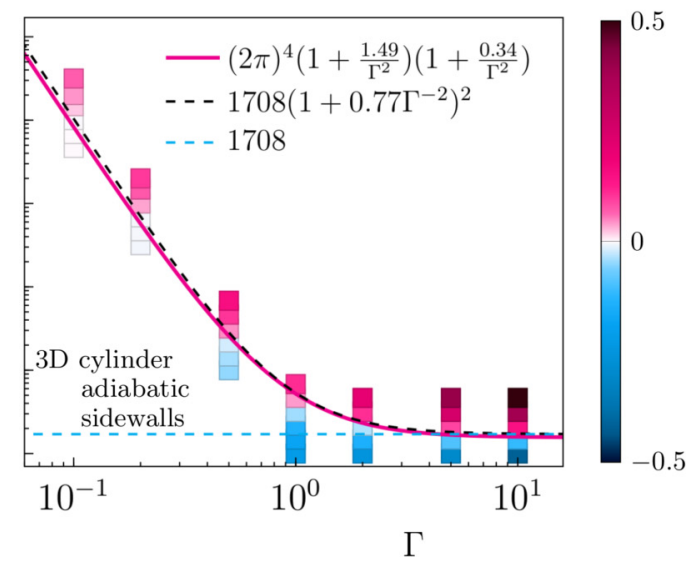

FIG. 6. Critical Rayleigh numbers $\mathrm{Ra}_{c, \Gamma}$ for the onset of convection, as functions of the domain aspect ratio $\Gamma$ : Linear growth rates (shown with colored vertically elongated boxes), obtained from numerical solutions of the linearized equations for fluctuations, initialized with random perturbations, and $(\mathrm{a}, \mathrm{b})$ the neutral stability curves (blue solid lines) as obtained from the classical linear stability analysis using the full spectrum of the normal modes, everywhere for no-slip boundary conditions for the velocity and $(a, b)$ two-dimensional rectangular domains and (c, d) three-dimensional cylindrical domains, with $(\mathrm{a}, \mathrm{c})$ conducting sidewalls and (b, d) adiabatic sidewalls; adapted from [59]. Black dashed lines show fits of the numerical solutions of the linearized equations, sought in the form $\operatorname{Ra}_{c, \Gamma} \approx 1708\left(1+C \Gamma^{-2}\right)^{2}$, with the best-fit constants $C$ shown in the corresponding legends. Pink continuous lines show the theoretical estimates that we have derived in this paper; see Eq. (54) for (a), Eq. (55) for (b), Eq. (67) for (c), and Eq. (68) for (d). Our theoretical estimates (pink solid lines) and the linear stability results obtained numerically from the linearized equations (black dashed lines) show excellent agreement. Blue dashed lines show a theoretical estimate $\operatorname{Ra}_{c, \infty}^{J} \approx 1708$ [23,24] for a laterally unbounded fluid layer, obtained from the linear stability analysis of the normal modes, restricted to the consideration of a single mode in the horizontal direction.

estimates of the critical Rayleigh numbers for the onset of convection, $\mathrm{Ra}_{c, \Gamma}$, as functions of $\Gamma$, which we have derived in the previous subsections, are shown with pink lines: Eq. (54) in Fig. 6(a), Eq. (55) in Fig. 6(b), Eq. (67) in Fig. 6(c), and Eq. (68) in Fig. 6(d). One can see that our theoretical estimates (pink solid lines) and the results of the numerically conducted linear stability analysis (black dashed lines) are in excellent agreement. 
In Fig. 6 one can also see Jeffreys' theoretical estimate $\operatorname{Ra}_{c, \infty}^{J} \approx 1708[23,24]$ for a laterally unbounded fluid layer, shown with horizontal blue dashed lines. As we know, this theoretical estimate, $\mathrm{Ra}_{c, \infty}^{J} \approx 1708$, was derived using the linear stability approach and under the assumption that the first unstable mode in the horizontal direction can be described by a single perturbation wave in a form $\sim \exp \left(i k_{x} x+i k_{y} y+\sigma t\right)$, which is the same for every component of the flow: the vertical component of the velocity, the horizontal component of the velocity, and the deviation of the temperature from its linear profile. This is actually a quite restrictive requirement and in fact contradicts the boundary conditions at the sidewalls, which are no-slip for the velocity (Dirichlet boundary conditions) and adiabatic for the temperature (Neumann boundary conditions). When the aspect ratio of the container $\Gamma$ becomes very large, the influence of the sidewalls reduces, and, therefore, the difference between Jeffreys' estimate for an infinite layer, $\operatorname{Ra}_{c, \infty}^{J} \approx 1708$ [23,24], and our estimate, $\operatorname{Ra}_{c, \Gamma}$ for $\Gamma \rightarrow \infty$, becomes smaller, however, not zero, as the set of possible solutions of the linearized equations was restricted to a single wave in the horizontal directions in the case of $\mathrm{Ra}_{c, \infty}^{J}$.

For $\Gamma \lesssim 4.4$, the value of $\mathrm{Ra}_{c, \Gamma}$ becomes larger than the Jeffrey's value $\operatorname{Ra}_{c, \infty}^{J} \approx 1708$ [23,24]. Both curves certainly deviate strongly in the limit $\Gamma \rightarrow 0$, where $\operatorname{Ra}_{c, \Gamma}$ grows as $\sim \Gamma^{-4}$, while $\operatorname{Ra}_{c, \infty}^{J}$ is just constant. In the other limit, $\Gamma \rightarrow \infty$, the value of $\operatorname{Ra}_{c, \Gamma \rightarrow \infty}=(2 \pi)^{4}$ for confined geometries is about $8.8 \%$ smaller than $\operatorname{Ra}_{c, \infty}^{J} \approx 1708$.

As we have already discussed, for laterally confined containers it is not sufficient to consider a single mode in the horizontal direction, and in this respect, the numerically conducted linear stability analysis, which admits any combinations of the modes in the horizontal directions, becomes more reliable and its results are more realistic than those of any theoretical approach based on an analysis of the linear stability of a single-mode perturbation in the horizontal direction.

Figure 6 demonstrated an excellent support of our theoretical results by the numerically obtained linear stability data from [59]. To have a closer look, let us also consider a ratio of the theoretical $\mathrm{Ra}_{c, \Gamma}$ and the fits of the $\mathrm{Ra}_{c, \Gamma}$ obtained numerically, which we present in Fig. 7. In Fig. 7(a) the fits of the numerical data are sought in a form $\mathrm{Ra}_{c, \Gamma} \approx 1708\left(1+C / \Gamma^{2}\right)^{2}$, while in Fig. 7(b) they are sought in a form $\operatorname{Ra}_{c, \Gamma} \approx(2 \pi)^{4}\left(1+C / \Gamma^{2}\right)^{2}$, with fitting constants $C$, specified in the legend.

In the limit $\Gamma \rightarrow \infty$, the ratio of the theoretical to numerical $\operatorname{Ra}_{c, \Gamma}$ converges to $(2 \pi)^{4} / 1708 \gtrsim$ 0.91, showing a small deviation between the theoretical and numerical data, if the numerical data are fitted in the form $1708\left(1+C / \Gamma^{2}\right)^{2}$; see Fig. 7(a). However, when the numerical data are fitted in the form $(2 \pi)^{4}\left(1+C / \Gamma^{2}\right)^{2}$, the ratio of the theoretical to numerical $\operatorname{Ra}_{c}, \Gamma$ clearly converges to 1 in the same limit $\Gamma \rightarrow \infty$, showing excellent agreement of the theoretical estimates and numerical linear stability data in that limit; see Fig. 7(b).

For very small $\Gamma$, the deviation between the numerical and theoretical data is larger, as one can see in Fig. 7. However, one should notice that within two orders of magnitude in small $\Gamma$, the values of the critical Rayleigh numbers, $\mathrm{Ra}_{c, \Gamma}$, range over about eight orders of magnitude, since in the limit $\Gamma \rightarrow 0$, the values of $\operatorname{Ra}_{c}, \Gamma$ grow as $\sim \Gamma^{-4}$. Thus, the accuracy of the numerically obtained critical Rayleigh numbers for the onset of convection unavoidably reduces with decreasing $\Gamma$. The ratio of the theoretical to numerical $\mathrm{Ra}_{c, \Gamma}$, presented in Fig. 7, is quite sensitive to the accuracy of the numerical data and the way they are fitted. In Fig. 7 one can also see, how a variation of the fitting coefficient by only 5\% (shown with dashed lines of the corresponding color) changes the ratio between the theoretical and numerical values of $\mathrm{Ra}_{c, \Gamma}$.

To complete the picture, in Fig. 8 we show the ratio between the plain numerically obtained linear stability data and our theoretical estimates, without applying any fitting. Again, taking into account that the values of the critical Rayleigh number for the onset of convection vary within about 5 orders of magnitude in the considered range of $\Gamma$, we conclude that our theoretical estimates indeed are in good agreement with the linear stability analysis.

Already for Rayleigh numbers slightly larger than the critical one, the flow can be represented by several modes of similar eigenvalues. The spectrum of possible states is broader for larger aspect ratios. In Fig. 5 one can see the temperature distributions in the convective flows close to the onset of 
(a)

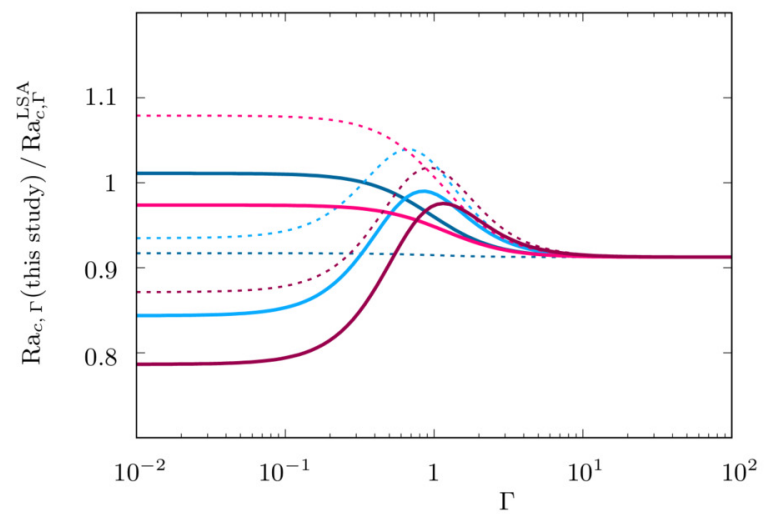

$2 \mathrm{D}$, conducting sidewalls, where LSA data [4] fitted as:

$-\quad \operatorname{Ra}_{c, \Gamma}^{\mathrm{LSA}}=1708\left(1+C / \Gamma^{2}\right)^{2}, C \approx 0.95$,

- . - as above, with $5 \%$ correction of the factor $C$

2D, adiabatic sidewalls, where LSA data [4] fitted as:

$-\operatorname{Ra}_{c, \Gamma}^{\mathrm{LSA}}=1708\left(1+C / \Gamma^{2}\right)^{2}, C \approx 0.52$,

- . as above, with $5 \%$ correction of the factor $C$.

3D, cylinder, conducting sidewall; LSA data [4] fitted as:

$\longrightarrow \operatorname{Ra}_{c, \Gamma}^{\mathrm{LSA}}=1708\left(1+C / \Gamma^{2}\right)^{2}, C \approx 1.44$,

- . - as above, with $5 \%$ correction of the factor $C$.

3D, cylinder, adiabatic sidewall; LSA data [4] fitted as:

$-\operatorname{Ra}_{c, \Gamma}^{\mathrm{LSA}}=1708\left(1+C / \Gamma^{2}\right)^{2}, C \approx 0.77$

- - - as above, with $5 \%$ correction of the factor $C$.

(b)

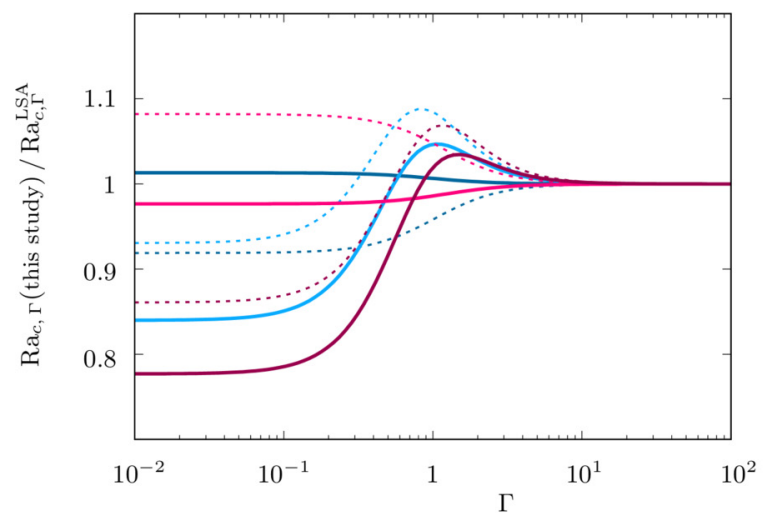

$2 \mathrm{D}$, conducting sidewalls, where LSA data [4] fitted as:

$-\operatorname{Ra}_{c, \Gamma}^{\mathrm{LSA}}=(2 \pi)^{4}\left(1+C / \Gamma^{2}\right)^{2}, C \approx 0.99$,

- - - as above, with $5 \%$ correction of the factor $C$.

2D, adiabatic sidewalls, where LSA data [4] fitted as:

$-\operatorname{Ra}_{c, \Gamma}^{\mathrm{LSA}}=(2 \pi)^{4}\left(1+C / \Gamma^{2}\right)^{2}, C \approx 0.55$,

- . - as above, with $5 \%$ correction of the factor $C$.

3D, cylinder, conducting sidewall; LSA data [4] fitted as:

$-\operatorname{Ra}_{c, \Gamma}^{\mathrm{LSA}}=(2 \pi)^{4}\left(1+C / \Gamma^{2}\right)^{2}, C \approx 1.50$,

- - - as above, with $5 \%$ correction of the factor $C$.

3D, cylinder, adiabatic sidewall; LSA data [4] fitted as:

$-\operatorname{Ra}_{c, \Gamma}^{\mathrm{LSA}}=(2 \pi)^{4}\left(1+C / \Gamma^{2}\right)^{2}, C \approx 0.81$,

- - - as above, with $5 \%$ correction of the factor $C$.

FIG. 7. (a) Solid lines: ratios of the critical Rayleigh numbers $\operatorname{Ra}_{c, \Gamma}$ for the onset of convection, as estimated in this study, and $\mathrm{Ra}_{c, \Gamma}$ as obtained from numerical solutions of the linearized equations for fluctuations (numerical linear stability analysis) [59], plotted versus the container aspect ratio $\Gamma$. The numerical linear stability data are represented by their fits sought in a form $\mathrm{Ra}_{c, \Gamma}^{\mathrm{LSA}} \approx 1708\left(1+C / \Gamma^{2}\right)^{2}$, with the best-fit constants $C$ shown in the legends here and in Fig. 6. $\mathrm{Ra}_{c, \Gamma}$ of this study are taken from Eq. (54) for twodimensional rectangular domains with conducting sidewalls, Eq. (55) for two-dimensional rectangular domains with adiabatic sidewalls, Eq. (67) for three-dimensional cylindrical domains with conducting sidewalls, and Eq. (68) for three-dimensional cylindrical domains with adiabatic sidewall. Everywhere no-slip boundary conditions for the velocity are considered. Dashed lines show similar data as the continuous ones but with a 5\% correction of the fitting coefficients in the numerical linear stability data. Panel (b) is as (a), but the numerical linear stability data are represented by their fits sought in a form $\operatorname{Ra}_{c, \Gamma}^{\mathrm{LSA}} \approx(2 \pi)^{4}\left(1+C / \Gamma^{2}\right)^{2}$, with the best-fit constants $C$ shown in the legend. Taking into account a very broad range of the considered aspect ratios $\Gamma$ and a certain inaccuracy of the numerical linear stability analysis data and of their fits, one can conclude that our theoretical estimates and the numerically obtained linear stability data [59] are in good agreement.

convection in parallelepiped and cylindrical containers (shown in, respectively, the upper and lower rows of Fig. 5) with adiabatic sidewalls, for different aspect ratios $\Gamma$. These thermal isosurfaces are numerical solutions of the linearized governing equations. One can compare horizontal structures of these modes, obtained in the linear stability study, with the theoretical ones shown in Fig. 2. A clear similarity is seen between Figs. 5(h)-(k) and Fig. 2(d) for $\Gamma \leqslant 1$, between Fig. 5(l) and Fig. 2(e) for $\Gamma \approx 2$ and between Fig. $5(\mathrm{~m})$ and Fig. $2(\mathrm{f})$ for $\Gamma \approx 5$. 


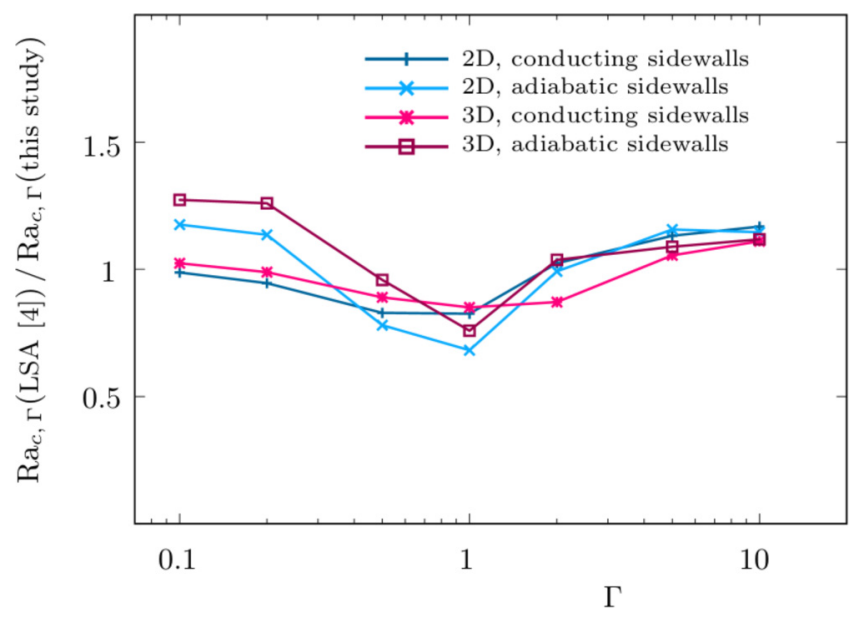

FIG. 8. Ratios of the critical Rayleigh numbers $\mathrm{Ra}_{c, \Gamma}$ for the onset of convection, as obtained from numerical solutions of the linearized equations for fluctuations (numerical linear stability analysis) [59], and $\mathrm{Ra}_{c, \Gamma}$ as estimated in this study, plotted versus the container aspect ratio $\Gamma$. The numerical linear stability data are shown as is, without any fitting procedure. The values of $\mathrm{Ra}_{c, \Gamma}$ of this study are taken from Eq. (54) for two-dimensional rectangular domains with conducting sidewalls, Eq. (55) for two-dimensional rectangular domains with adiabatic sidewalls, Eq. (67) for three-dimensional cylindrical domains with conducting sidewalls, and Eq. (68) for three-dimensional cylindrical domains with adiabatic sidewall. Everywhere no-slip boundary conditions for the velocity are considered. Taking into account that within the considered $\Gamma$-range, the values of $\mathrm{Ra}_{c, \Gamma}$ vary within 5 orders of magnitude, one can agree that our theoretical estimates and the numerically obtained linear stability data [59] are in good agreement.

\section{Comparison with measurements}

Convection by its onset has been mainly studied for wide containers (see [6,21]). Here, the measurements of the critical Rayleigh numbers and pattern formation have been of particular interest. It is quite understandable that for very large aspect ratios one expects the critical Rayleigh number about 1708 and the flow pattern with straight rolls, as predicted by the linear stability theory for an infinite fluid layer, assuming that the sidewalls contribution is little in the case $\Gamma \gg 1$.

The critical Rayleigh number for the onset of convection is associated with a departure of the Nusselt number from unity. Thus, based on measurements in cylindrical containers with the aspect ratio $\Gamma$ in the range $15.2 \lesssim \Gamma \lesssim 136.6$, filled with different fluids, Silveston [65] reported $\mathrm{Ra}_{c, \Gamma}=1700 \pm 51$, in agreement with calculations by Jeffreys [23] for an infinite fluid layer. These measurements were also the main experimental study to refer to Chandrasekhar [25]. Although the declared critical Rayleigh number in the study [65] is $\mathrm{Ra}_{c, \Gamma}=1700 \pm 51$, at least in some cases, a fluid motion started prior to this value of the Rayleigh number. For example, Fig. 9(d) of [65] shows a certain flow pattern for $\mathrm{Ra}=1700$ and $\Gamma \approx 28.3$. This pattern becomes more pronounced for larger Rayleigh numbers, but also can be quite clearly traced on Fig. 9(c) for Ra $=1640$. The remains of this pattern can be recognized even on Fig. 9(b) of [65] for Ra $=1590$. Our estimate (68) for $\Gamma=28.3$ suggests that convection starts at $\mathrm{Ra}_{c, \Gamma} \approx 1562$. Measurements in water, conducted by Behringer and Ahlers [64], reported $\operatorname{Ra}_{c, \Gamma}=1599 \pm 240$ for $\Gamma \approx 9.44$ and $\operatorname{Ra}_{c, \Gamma}=1694 \pm 250$ for $\Gamma \approx 4.15$. The corresponding estimates that follow from (68) are $\operatorname{Ra}_{c, \Gamma} \approx 1591$ for $\Gamma=9.44$ and $\mathrm{Ra}_{c, \Gamma} \approx 1694$ for $\Gamma=4.15$.

For large aspect ratios, a deviation of the Nusselt number from unity can become recognizable in experiments only when bulk convection sets in, as any activity localized near the sidewalls can significantly increase only the local Nusselt number, but not the averaged over the whole horizontal cross-sectional heat transport. Thus if by the onset of fluid motion the flow is localized near 
(a) $\xi_{d}=1, \xi_{w}=0$

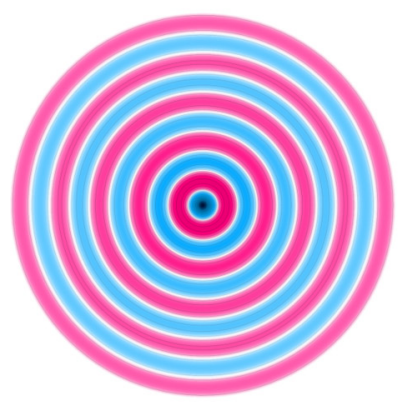

(d) $\xi_{d}=0.4, \xi_{w}=0.6$

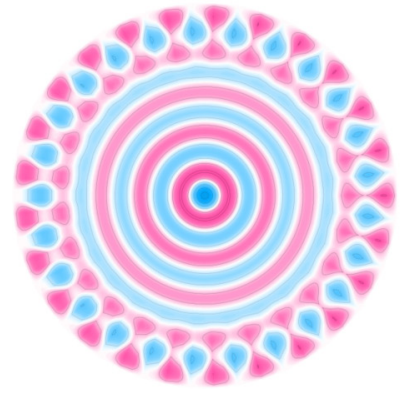

(b) $\xi_{d}=0.8, \xi_{w}=0.2$

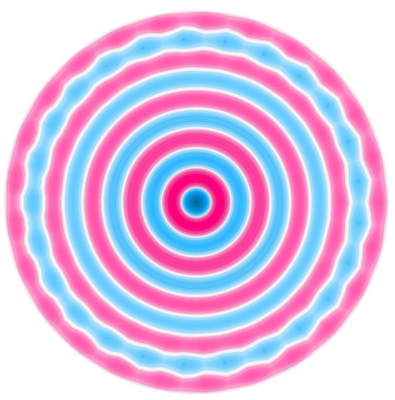

(e) $\xi_{d}=0.2, \xi_{w}=0.8$

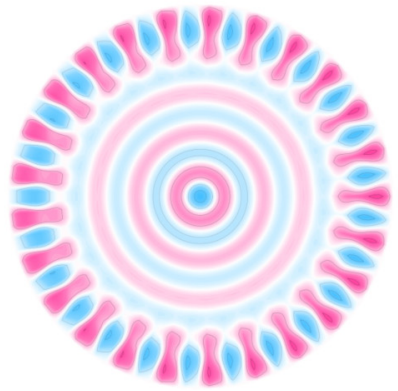

(c) $\xi_{d}=0.6, \xi_{w}=0.4$

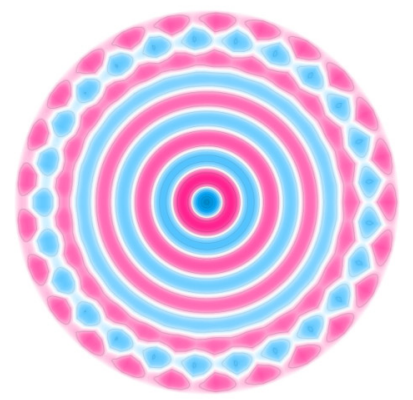

(f) $\xi_{d}=0, \xi_{w}=1$

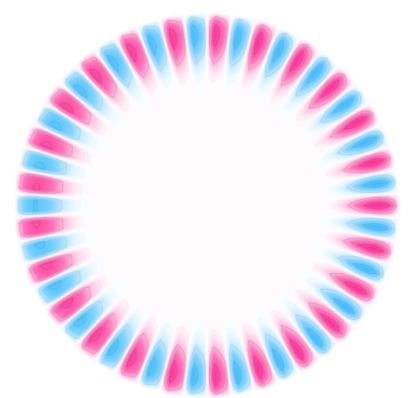

FIG. 9. Mixed modes, according to Eq. (95), which are combinations of the donut mode and wall mode that have similar eigenvalues. The contributions of the donut mode and wall mode are reflected in the prefactors $\xi_{d}$ and $\xi_{w}$, respectively.

the sidewall, it is not recognizable in the measurements of the Nusselt number, and therefore is interpreted as a "subcritical" fluid motion; see, for example, Croquette et al. [66]. However, if under the onset of convection we understand the onset of any fluid motion (as we do here) and not a visible increase of the total heat transport compared to the steady state (as it is usually done in the measurements of the critical Rayleigh numbers), we should admit that the true critical Rayleigh number is lower than the one derived from the measurements, as soon as a "subcritical" flow motion in the same setup was observed. Here one can also recollect that in the Oberbeck-Boussinesq Rayleigh-Bénard convection there is no subcritical instability.

Flow patterns that are observed in experiments in confined parallelepiped and cylindrical containers of very large aspect ratios close to the onset of convection are quite remarkable. In parallelepiped domains with $\Gamma_{y}<\Gamma_{x}$, the rolls always develop along the short side of the heated or cooled plates (see [67]), which is in agreement with Eq. (56). Alignment of the rolls along the long side would need larger $\mathrm{Ra}_{c}, \Gamma$, i.e., proportional to $\left(1+\Gamma_{y}^{-2}\right)$ and not to $\left(1+\Gamma_{x}^{-2}\right)$, in accordance with Eq. (56). For square plates, $\Gamma_{y}=\Gamma_{x}$, the flow structure can include rolls aligned orthogonally to each other, along the $x$ - and $y$-directions.

In experiments, it is also observed that the rolls in confined containers usually try to align orthogonally to the sidewalls. This holds also for circular cylindrical containers with adiabatic and conducting sidewalls. Even in very large aspect ratio containers, when the rolls are straight or look like nested annular rolls in the core part of the domain, the rolls try to change their orientation near the sidewall in such a way that they approach the sidewall at a right angle (see, e.g., $[66,68]$ ). Although the role of the sidewalls weakens with increasing Rayleigh number, the flow pattern close to the onset of convection is much affected by the sidewalls. One can reduce the effect of the sidewalls by certain manipulations of the conditions at the plates, next to the sidewall. For example, 
one can let a certain gap between the top plate and a sidewall and thus support the development of different three-dimensional flow structures next to the sidewall, which let the rolls approach the sidewalls at any angle (see [21,69]). Another option is to achieve the thermal properties of the sidewall very close to those of the working fluid and thus reduce the sidewall effects, as was done by Meyer et al. [70]. With this or that way to reduce the influence of the sidewalls in large aspect ratio containers for Rayleigh numbers close to the critical one, one can mimics a laterally unconfined fluid layer and enjoy seeing the straight rolls [21,69,71] and cellular patterns [70] as predicted by the linear stability theory for an infinite layer.

However, without suppression of the sidewall influence in wide cylindrical containers, at the very onset of convection one usually observes concentric annular rolls that fill the core part of the container and might show some irregularity near the sidewalls (see $[6,21,68,70,72-74])$. "The pattern clearly reflects the geometry of the walls, and the images illustrate how this pattern spreads. It develops in the region adjacent to the wall and moves inward filling the cell," as reported by Meyer et al. [70]. This axisymmetric flow structure becomes less stable as soon as the sidewall influence is reduced (either by manipulation of the boundary conditions, as, e.g., in [70], or by increasing the Rayleigh number), which might lead, in particular, to the formation of other patterns like straight-roll patterns [68] or eccentric annular rolls patterns [75].

How can one interpreted in the light of the above theory the principle flow structure that one observes in experiments by the very onset of convection in wide cylindrical containers, that is, the concentric annular rolls, with possible irregularity near the sidewalls? For this we need to recollect the property of degenerate eigenvalues, discussed in Secs. II D and II E, Fig. 3 and Fig. 4. The reference "two-by-two" mode with $n=1, k=1$ and $n_{z}=2$ [like in Figs. 3(a) and 3(b) and Figs. 4(a) and 4(b)] can have the same or very similar eigenvalue as a "wall mode" [like in Figs. 3(b) and 3(d)] or as a "donut mode" [like in Figs. 4(b) and 4(d)].

Let us consider a thought example of a wide cylindrical container with a conductive sidewall. The leading eigenmode by the onset of convection has a form

$$
J_{n}\left(2 j_{n k} r / D\right) \cos (n \phi) \sin \left(\pi n_{z} z / H\right)
$$

and corresponds to the eigenvalue

$$
\lambda=4 j_{n k}^{2} / D^{2}+\pi^{2} n_{z}^{2} / H^{2}
$$

with certain integers $n$ and $k$ and $n_{z}=1$. This mode should be energetically similar to the "two-bytwo" mode with $n=1, k=1$, and $n_{z}=2$, so that they both correspond to

$$
\operatorname{Ra}_{c, \Gamma}=H^{4}\left(4 j_{n k}^{2} / D^{2}+\pi^{2} n_{z}^{2} / H^{2}\right)^{2} \approx 1596 .
$$

This leads to the following relation:

$$
j_{n k} \approx\left(\frac{3}{4} \pi^{2} \Gamma^{2}+j_{11}^{2}\right)^{1 / 2},
$$

which for $\Gamma$ about 11.17 gives $j_{n k} \approx 30.6$. The closest to this value is $j_{n k}$ for $n=0$ and $k=10$ $\left(j_{0,10} \approx 30.6\right)$, which suggests that the "donut mode" with concentric annular rolls is the first candidate for the leading mode by the onset of convection in the considered geometry. This mode is plotted in Fig. 9(a).

When the Rayleigh number is slightly increased and achieves the value $\mathrm{Ra} \approx 1619$, so that

$$
\varepsilon \equiv\left(\mathrm{Ra}-\mathrm{Ra}_{c, \Gamma}\right) / \mathrm{Ra}_{c, \Gamma} \approx 0.014,
$$

and the system still remains in the linear regime, but can sustain modes with a little bit larger but similar eigenvalues with $j_{n k} \approx 30.7$, one can observe a mixture of the above donut mode $(n=0$, $k=10, n_{z}=1$ ) with the wall mode for $n=25, k=1$ and $n_{z}=1$, since for this wall mode $j_{25,1} \approx$ 30.7. This wall mode is shown in Fig. 9(f). Thus in this case a mixture of the two modes, which is 
proportional to

$$
\propto \xi_{d} J_{0}\left(2 j_{0,10} r / D\right) \sin (\pi z / H)+\xi_{w} J_{25}\left(2 j_{25,1} r / D\right) \cos (25 \phi) \sin (\pi z / H),
$$

might be possible.

Figure 9 shows some of those mixed modes, for different combinations of the prefactors $\xi_{d}$ and $\xi_{w}$ that indicate the contributions of the donut or wall mode, respectively. One can see a clear similarity between the donut mode in Fig. 9(a) and concentric rolls obtained in the experiments; see, for example, Ahlers et al. [72] (Fig. 1, $t=-0.2$ ), Meyer et al. [70] (Fig. 1), and Hu et al. [68] [Fig. 3(a)]. A combination of the donut and wall modes like in Fig. 9(e) shows similarity with the experimentally obtained patterns that include concentric rolls in the core part of the domain and transverse short rolls near the sidewalls; see examples in Croquette et al. [66] (Fig. 3), Ahlers et al. [72] (Fig. 1, $t=0.3$ ), and $\mathrm{Hu}$ et al. [68] [Fig. 3(b)]. Of course, this similarity is only qualitative, since the aspect ratios in these measurements are different as well as the thermal boundary conditions at the sidewall: in the experiments, they are rather adiabatic, while here, for simplicity, we consider a conductive sidewall.

Analogously, with further increase of the Rayleigh number, modes with $j_{n k} \approx 30.8$ can occur, and in particular, the mode with $n=9$ and $k=6$ becomes possible, as $j_{9,6} \approx 30.8$. With that mixed modes can take the form

$$
\begin{aligned}
\propto \xi_{d} J_{0}\left(2 j_{0,10} r / D\right) \sin (\pi z / H)+ & \xi_{w} J_{25}\left(2 j_{25,1} r / D\right) \cos (25 \phi) \sin (\pi z / H) \\
& +\xi J_{9}\left(2 j_{9,6} r / D\right) \cos (9 \phi) \sin (\pi z / H) .
\end{aligned}
$$

Figure 10 shows some of these modes for $\xi=1-\xi_{d}-\xi_{w}$. With increasing Rayleigh number, however, not only more and more new modes occur, but also the nonlinearity of the governing equations comes into play, which all together influences the evolution of the flow pattern.

As already discussed, with the dominance of the wall modes like in Figs. 9(e) and 9(f) or in Figs. 10(d) and 10(h), the total heat transport enhancement due to convection is negligible. This is because the area of the strongest fluid motion near the sidewall is small. Therefore the regime of the wall mode dominance is usually identified in the experiments as a "subcritical" regime (which strictly speaking, as we do know, does not exist in the Oberbeck-Boussinesq case).

We end this subsection with a proposition that in large aspect ratio containers, the probability that convection starts from the sidewall increases with growing aspect ratio $\Gamma$. This means that the wall modes should generally appear prior to the bulk (donut-like) modes. This can be explained as follows. The roots $j_{n k}$ of the Bessel function $J_{n}$ which appear in the leading mode of the form (90) grow with the aspect ratio $\Gamma$, according to (93).

In the limit $k \rightarrow \infty$, the roots $j_{0 k}$ of the Bessel function $J_{0}$, which appear in the donut mode of the form (90), follow the Stokes asymptotic expansions [76]

$$
j_{0 k}=\pi k-\pi / 4+O\left(k^{-1}\right), \quad k \rightarrow \infty .
$$

On the other hand, in the limit $n \rightarrow \infty$, the first root $j_{n 1}$ of the Bessel function $J_{n}$, which appears in the wall mode of the form (90), follows the McMahon asymptotic expansion [77]

$$
j_{n 1}=n-C n^{1 / 3}+O\left(n^{-1 / 3}\right), \quad n \rightarrow \infty,
$$

with $C \approx 1.856$. This means that asymptotically, the neighboring roots $j_{0 k}$ are separated by $\pi$, while the neighboring roots $j_{n 1}$ are separated by one; in other words, the roots $j_{n 1}$ are distributed much denser than the roots $j_{0 k}$. This makes clear that most probably a certain value $j_{n 1}$ (that corresponds to a wall $n$-mode) rather than a certain value $j_{0 k}$ (that corresponds to a donut bulk $k$-mode) will be the nearest one to a given large value of $\left(\frac{3}{4} \pi^{2} \Gamma^{2}+j_{11}^{2}\right)^{1 / 2}$, which is dictated by the critical Rayleigh number; see relation (93). Thus, in most cases of $\Gamma \gg 1$, the wall modes can provide the best match between the critical Rayleigh number and the eigenvalues of the realizable modes. The presence of the wall modes is the reason for the tendency of different roll-shaped flow patterns to approach the sidewalls at right angles, like, for example, is shown in Figs. 9(e) and 9(f) or Figs. 10(d) and 10(h). 
(a) $\xi_{d}=0, \xi_{w}=0$

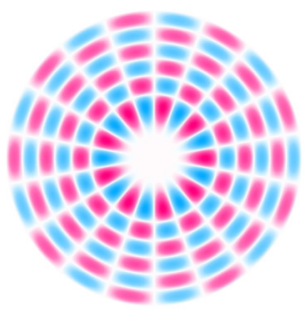

(e) $\xi_{d}=0.25, \xi_{w}=0$

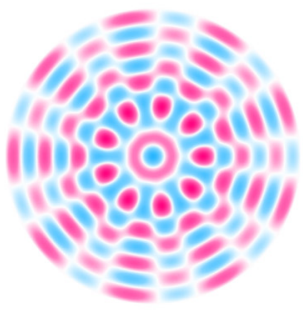

(i) $\xi_{d}=0.5, \xi_{w}=0$

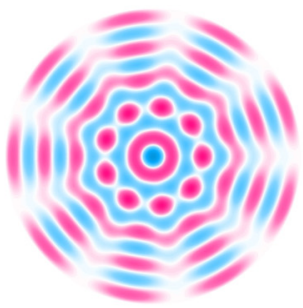

(b) $\xi_{d}=0, \xi_{w}=0.25$

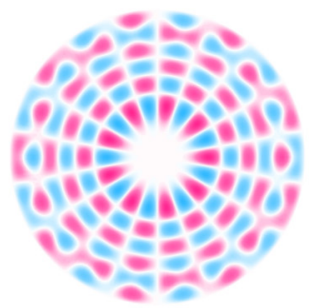

(f) $\xi_{d}=0.25, \xi_{w}=0.25$

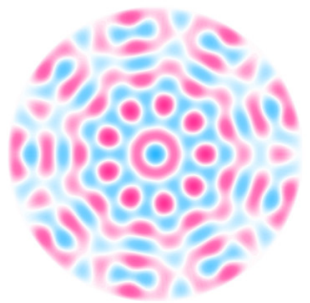

(j) $\xi_{d}=0.5, \xi_{w}=0.25$

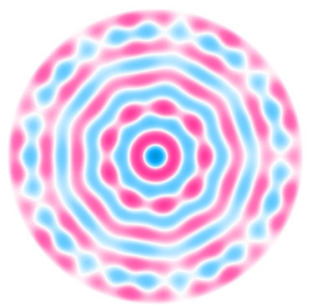

(c) $\xi_{d}=0, \xi_{w}=0.5$

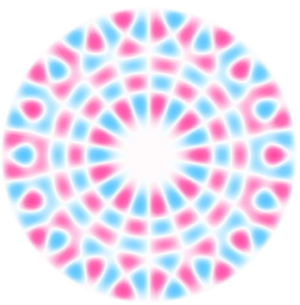

(g) $\xi_{d}=0.25, \xi_{w}=0.5$

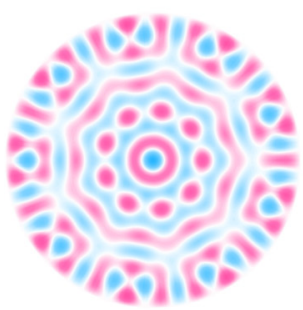

(k) $\xi_{d}=0.5, \xi_{w}=0.5$

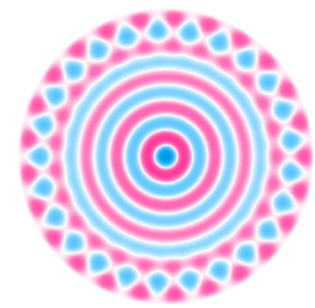

(d) $\xi_{d}=0, \xi_{w}=0.75$

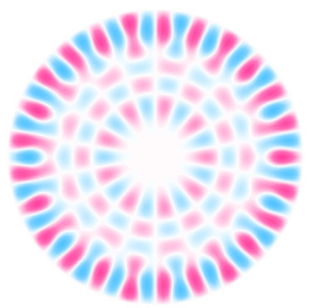

(h) $\xi_{d}=0.25, \xi_{w}=0.75$

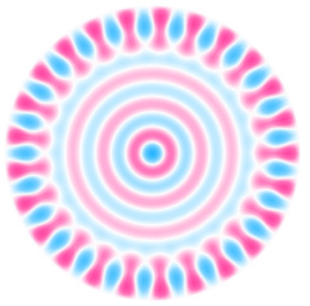

(l) $\xi_{d}=0.75, \xi_{w}=0$

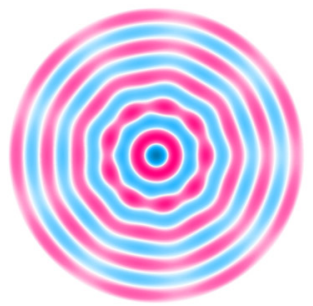

FIG. 10. Mixed modes, according to Eq. (96), which are combinations of three modes that correspond to similar eigenvalues. The contributions of the donut mode and wall mode are reflected in the prefactors $\xi_{d}$ and $\xi_{w}$, respectively.

With this respect, we can also refer to the experimental studies by Croquette et al. [66], where the radial profiles of the measured modes are presented (Fig. 5 of [66]) and the structure of the mixed mode (Fig. 3 of [66]) is sketched. The shown radial profiles clearly demonstrate that in the measurements, only the onset of the bulk modes is associated with the supercritical regime [Figs. 5(c)-5(g) of [66]]. In what the authors call as "subcritical" regime [Figs. 5(a) and 5(b) of [66]], a fluid motion is also observed, however, with the maximal amplitude near the sidewalls only. This is in agreement with our proposition.

We can remark that the onset of wall modes prior to bulk modes is similar to a certain extent to the occurrence of the wall modes in rotational Rayleigh-Bénard convection prior to the onset of bulk convection (see [78-89]). When a cylindrical Rayleigh-Bénard cell is rapidly rotated along its axis, convection is certainly suppressed, and larger, compared to the nonrotating case, Rayleigh numbers should be achieved in order to enforce a fluid motion. The critical Rayleigh number for the onset of convection in the rotational case essentially depends on the rotation rate [25]. However, in rotational Rayleigh-Bénard convection, the sidewalls always play a destabilizing role and convection first occurs close to the sidewalls in a form of periodic wall modes, while the bulk modes occur later, for larger Rayleigh number. (We will discuss rotational Rayleigh-Bénard convection later again, in Sec. V.)

Very similar stable wall mode states, which are traveling waves along the sidewall of a cylindrical container, one can also observe at the very onset of nonrotating Rayleigh-Bénard convection in 
mixtures; see, for example, "traveling waves surrounding pure conduction" in [90] and Fig. 3 there. In these experiments by Lerman et al. [90], where an ethanol-water mixture was used as a working fluid, short rolls, attached almost orthogonally to the sidewall, traveled in the clockwise direction.

\section{J. Container distortion effect and imprecise implementation of the boundary conditions}

Finally, at the end of this section, let us briefly discuss some specific features of the eigenmodes of the Laplace operator in wide domains $(\Gamma \gg 1)$, which might give an additional reason why in experiments and numerical simulations of Rayleigh-Bénard convection in wide but anyway laterally confined containers, convection can start from the sidewalls towards the bulk and not simultaneously in the entire domain as it is supposed to be in an unbounded fluid layer. This also concerns the first eigenvalues of the Laplace operator and, hence, the critical Rayleigh number for the onset of convection in wide domains with imperfect sidewall boundary conditions, for which the Laplace eigenvalues and, therefore, also the critical Rayleigh number, might slightly deviate from the theoretical estimates for this considered geometry of a wide Rayleigh-Bénard cell.

Let us consider wide cylindrical containers $(\Gamma \gg 1)$ with adiabatic sidewalls, where these sidewalls are not perfectly straight but somehow slightly distorted. (Such a situation is very realistic in experimental facilities.) To estimate the critical Rayleigh number for the onset of convection in such domains, we need to calculate the first eigenvalues of the Laplace operator for these domains, with mixed boundary conditions, Dirichlet at the plates and Neumann at the distorted sidewalls. Nazarov et al. [91,92] studied the behavior of the eigenfunctions of the Laplace operator with such mixed boundary conditions in thin cylindrical domains subjected to a certain tiny distortion of the plates and/or sidewalls and showed, in particular, that these tiny geometrical manipulations of the cylinder sidewall for $\Gamma \gg 1$, can lead to localization of the first eigenfunctions in the vicinity of the sidewall, with an exponential decay toward the center of the domain [93]. Such phenomenon of localization and exponential decay of the eigenfunctions of the Laplace operator has been reported for various perturbations of cylindrical domains; see Grebenkov and Nguyen [58]. The corresponding leading eigenfunctions are known as "trapped modes," as they are localized at some preferable places—near the sidewall in the considered case. (See also Friedlander and Solomyak [94,95] concerning the spectrum of Dirichlet eigenfunctions of the Laplace operator for narrow domains of different profiles.) Thus, small perturbations of the sidewalls of wide cylindrical containers or of the boundary condition prescribed on them, can lead to formation of the trapped modes, which are localized near the sidewall—and, therefore, we can call them as "wall modes" as well—and which exponentially decay toward the core of the cylinder.

For us, this means that for the case $\Gamma \gg 1$, any numerical or experimental distortion of the sidewall or plates, or a little local change of the boundary conditions near or at the sidewalls can lead to a formation of the wall modes, i.e., the first eigenmodes that are located near the sidewall and exponentially decay towards the bulk. These wall modes would in any case appear prior to the bulk modes that evolve in the entire domain. With that one can expect that the critical Rayleigh number for the onset of these wall modes, $\mathrm{Ra}_{c}, \Gamma$, would be slightly smaller than the critical Rayleigh number for the onset of bulk convection in a perfect wide cylindrical container for the same large aspect ratio of the container $\Gamma \gg 1$.

\section{OPTIMAL CONTAINER SHAPE IN RAYLEIGH-BÉNARD EXPERIMENTS}

Now that we have quite accurate estimates of the critical Rayleigh number for the onset of convection, let us discuss the optimum of the convection cell shape that maximizes the range of the Rayleigh numbers studied experimentally or numerically. We will consider this problem as a purely mathematical one, assuming that the Boussinesq conditions can hold for any height of the convection cell and that there are no constraints on the laboratory space and technical feasibility. We restrict our consideration only to containers that have the same horizontal cross sections at any 
height $z, 0 \leqslant z \leqslant H$. This allows separation of variables in (a) the horizontal directions and (b) vertical direction.

By the optimal shape of the convection cell (under specified constraints) we understand such a shape that for any given Boussinesq fluid maximizes the order of magnitude in the Rayleigh numbers that can be achieved using this cell, relative to the onset of convection. In other words, the optimal container shape maximizes the ratio between the maximal achievable in the experiment Rayleigh number and the Rayleigh number for the onset of convection in the same container. We will first discuss the optimal shape of the plates (i.e., the optimality of the convection cell in the horizontal directions) and then the optimal height of the convection cell, for a given shape of the plates (i.e., the optimality of the convection cell in the vertical direction).

\section{A. Isoperimetric inequalities and optimal shape of the heated and cooled plates}

Neither shape nor size of the plates influences the Rayleigh number that can be achieved in the experiment. However, the plate shape influences the critical Rayleigh number for the onset of convection, $\mathrm{Ra}_{c, \Gamma}$. Indeed, as we have estimated in the previous section,

$$
\operatorname{Ra}_{c, \Gamma} \approx(2 \pi)^{4} \lambda_{u} \lambda_{\theta}
$$

where $\lambda_{u}$ and $\lambda_{\theta}$ are related to the lowest relevant eigenvalues of the Laplace operator in the considered domain for, respectively, the velocity and temperature boundary conditions. Due to the separation of variables, these $\lambda_{u}$, and $\lambda_{\theta}$ take the form

$$
\lambda_{u} \sim 1+c_{u} \Gamma^{-2} \text { and } \lambda_{\theta} \sim 1+c_{\theta} \Gamma^{-2},
$$

where the constants $c_{u}>0$ and $c_{\theta}>0$ depend on the plate shape (or the shape of the horizontal cross section of the container). These constants are related to the eigenvalues of the two-dimensional Laplace operator in a domain, which has a shape of the plate, and for the boundary conditions as those of the sidewall: Dirichlet (no-slip) for the velocity and Neumann or Dirichlet for the temperature, for adiabatic or thermally conducting sidewalls, respectively. Thus, in order to minimize the critical Rayleigh number for the onset of convection (99) by optimizing the shape of the plate, it is necessary to answer the question, in which two-dimensional (convex) domains the smallest relevant eigenvalues of the Laplace operator with the corresponding boundary conditions are minimized.

An answer to this question-which geometry provides the smallest Laplace eigenvalues with Dirichlet boundary conditions-was proposed with respect to a different subject, namely, for the theory of sound. This was done by Lord Rayleigh himself, after whom the here studied type of thermal convection and the main control parameter, the Rayleigh number, are named. In his theoretical study of sound [96], Lord Rayleigh evaluated the "relative frequency in certain calculable cases for the gravest tone of membranes under similar mechanical conditions and of equal area" and showed "the effect of a greater or less departure from the circular form" (see [96], Sec. 211 of Chapter IX). In other words, it was conjectured that among all clamped membranes of the same area and the same tension, the circular membrane produces the lowest fundamental frequency. Note that up to a constant that depends on the tension of the membrane and its mass density, the oscillation frequencies of the membrane are determined by the eigenvalues of the Laplace operator with Dirichlet boundary conditions in a domain that matches the shape of the membrane. Rayleigh's proposition was proved independently by Faber [97] and Krahn [98] and now is known as the Rayleigh-Faber-Krahn inequality that states that the circular shape of the domain minimizes the first eigenvalue of the Laplace operator with Dirichlet boundary conditions among all two-dimensional convex domains with the same area [56,57]. A similar result for Neumann boundary conditions was obtained by Szegö [99].

However, the assumption, that in the expansions of the characteristics of the flow by the onset of convection, in terms of the eigenfunctions of the Laplace operator, the first relevant eigenmode changes the sign, would require in most cases minimization of the second and not the first eigenvalue. Concerning the second eigenvalue of the Laplace operator with Dirichlet boundary 
conditions, Krahn [100] derived that its minimum among two-dimensional domains of the same area is achieved by the union of two identical circles. This does not help much with respect to the optimal shape of the heated and cooled plates in Rayleigh-Bénard experiments as we are essentially looking for an optimal plate shape candidate only among convex plane domains. A natural hypothesis, that a domain with a "stadium" shape (a convex hull of two identical tangent circles) minimizes among convex plane domains the second eigenvalue for the case of Dirichlet boundary conditions, was also refuted by Henrot and Oudet [101]. Unfortunately, the problem to find a convex planar domain with a given area, which minimizes the second eigenvalue of the Laplace operator with Dirichlet or Neumann boundary conditions seems to be still an open problem [58,102].

Good news is that a similar problem, to find a convex planar domain with not a given area but a given diameter of the domain, that minimizes the first eigenvalues of the Laplace operator with Dirichlet boundary conditions, was recently solved by Bogosel et al. [103]. (For a convex plane domain, the diameter is the largest distance between two opposite parallel lines, which are tangent to the domain boundary.) Bogosel et al. [103] even listed all 17 eigenvalues for which the circle-shaped domain is a minimizer. This list includes the first two eigenvalues.

With that we can conclude that among all plates of the same diameter, a circle-shaped plate is for sure the best choice to minimize the critical Rayleigh number for the onset of convection in containers with conducting sidewalls. This might not be the case for other constrains, for example, for other boundary conditions at the sidewall or when the area of the plates is fixed and not their diameter. However, as discussed above, there are some serious arguments supporting that the optimal shape of the heated and cooled plates is round or close to the "stadium" shape. In the next subsection, we will demonstrate, in particular, that for the case of adiabatic sidewalls of a Rayleigh-Bénard cell, the usage of circular plates always leads to smaller critical Rayleigh numbers for the onset of convection than the usage of any rectangular plates, if the area of the circular and rectangular plates are equal.

\section{B. Rectangular and circular plates}

In this subsection we will compare the critical Rayleigh numbers for the onset of convection in cylindrical and parallelepiped convection cells with adiabatic sidewalls, assuming that the formulas (56) and (68) are exact.

For a parallelepiped domain, among all $\Gamma_{y} \leqslant \Gamma_{x}$, the value of $\operatorname{Ra}_{c, \Gamma}$ is minimal if $\Gamma_{y}=\Gamma_{x}=\Gamma$; see Eq. (56). Thus, both in a cylinder and in an optimal parallelepiped, the critical Rayleigh number can be calculated as follows:

$$
\operatorname{Ra}_{c, \Gamma} \approx(2 \pi)^{4}\left(1+\frac{c_{u}}{\Gamma^{2}}\right)\left(1+\frac{c_{\theta}}{\Gamma^{2}}\right)
$$

with

$$
\begin{gathered}
c_{u}=1 \quad \text { and } c_{\theta}=0.5 \quad \text { for parallelepiped, } \\
c_{u} \approx 1.4876 \quad \text { and } c_{\theta} \approx 0.3435 \text { for cylinder, }
\end{gathered}
$$

where the values of $c_{u}$ and $c_{\theta}$ we have taken from Eqs. (56) and (68).

In Fig. 11 we compare our theoretical estimates for the cases of the optimal parallelepiped and cylinder. One can see that for the case of equal areas of the plates in a cylindrical and parallelepiped containers, the critical Rayleigh number is generally smaller for the cylinder (see pink curves in Fig. 11). This means that for a fixed area of the plates, a circular shape of the plates is closer to the optimum.

In the limit $\Gamma \rightarrow 0$, Eq. (101) is reduced to

$$
\mathrm{Ra}_{c, \Gamma} \approx(2 \pi)^{4} c_{u} c_{\theta} \Gamma^{-4}, \quad \Gamma \rightarrow 0
$$




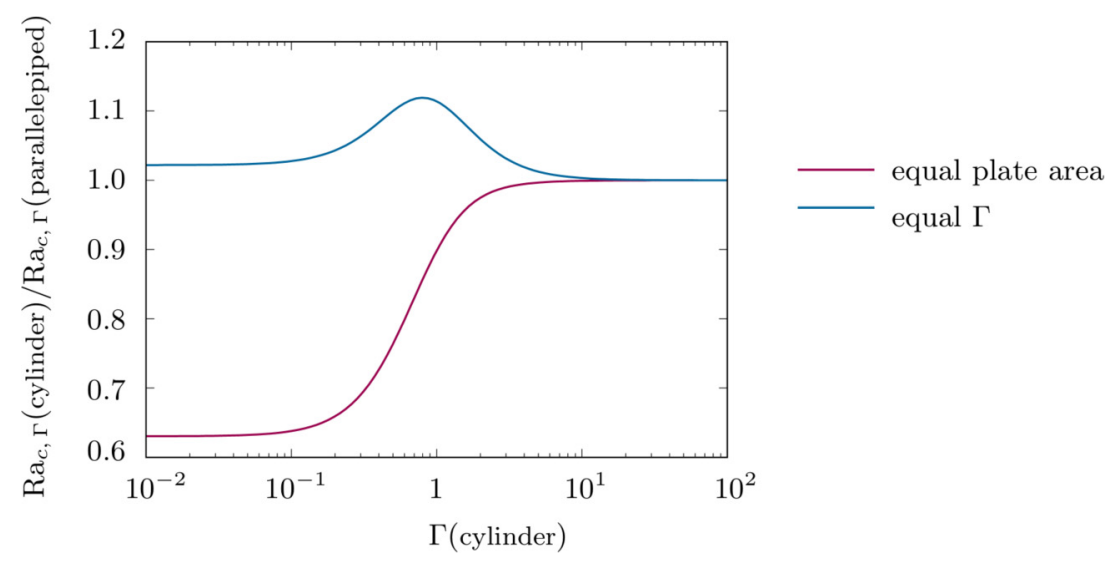

FIG. 11. Ratio of the critical Rayleigh numbers $\mathrm{Ra}_{c, \Gamma}$ for the onset of convection in a cylindrical container and in an optimal parallelepiped container with equal widths of the sidewalls $\left(\Gamma_{y}=\Gamma_{x}\right)$, for the cases of the same aspect ratio $\Gamma$ of the parallelepiped and cylindrical containers (blue line) or for the same area of the plates in the parallelepiped and cylindrical containers (pink line), as functions of the aspect ratio of the cylindrical container $\Gamma$. Everywhere, the boundary conditions are no-slip for the velocity at all walls and isothermal at the plates, and adiabatic at the sidewalls for the temperature. Calculations are according to Eqs. (101)-(103).

Substituting Eqs. (102)-(103) into Eq. (104), we obtain

$$
\operatorname{Ra}_{c, \Gamma}(\text { cylinder }) / \operatorname{Ra}_{c, \Gamma}(\text { parallelepiped }) \approx 1.02, \quad \Gamma \rightarrow 0 \quad(\text { for equal } \Gamma),
$$

which means that for the same aspect ratio of the cylindrical and parallelepiped convection cells, the critical Rayleigh number for the onset of convection in a cylinder is only $2 \%$ larger than in the optimal parallelepiped container (see blue dashed line in Fig. 11, for small $\Gamma$ ). We recall that here, following the tradition of the Rayleigh-Bénard community, under the aspect ratio of a parallelepiped domain we understand not the ratio of the length of the diagonal of a rectangular plate to the height of the cell, but the ratio of the longest length of the plate to the height of the cell.

To compare results for cylindrical and parallelepiped containers with the same area of the plates, for the latter case we need to substitute $\Gamma$ for $(\sqrt{\pi} / 2) \Gamma$ in Eq. (101). Thus, for a parallelepiped container, which has the same plate area as a cylindrical container of the aspect ratio $\Gamma$, we obtain

$$
\operatorname{Ra}_{c, \Gamma} \approx 128 \pi^{2} \Gamma^{-4}, \quad \Gamma \rightarrow 0 \quad\left(\Gamma_{x}=\Gamma_{y}=\frac{\sqrt{\pi}}{2} \Gamma\right) .
$$

Taking Eq. (106) for a parallelepiped container and Eqs. (104) and (103) for a cylinder, we conclude that in the limit of the cylinder aspect ratio $\Gamma \rightarrow 0$, the critical Rayleigh number for the onset of convection in a cylinder is significantly smaller than that for the optimal box with the equal area of the plates. More precisely,

$$
\operatorname{Ra}_{c, \Gamma}(\text { cylinder }) / \operatorname{Ra}_{c, \Gamma}(\text { parallelepiped }) \approx 0.63, \quad \Gamma \rightarrow 0 \quad \text { (for equal plate area); }
$$

see the pink solid line in Fig. 11, for small $\Gamma$.

In Fig. 11 we compared the cylindrical and the optimal parallelepiped containers, i.e., those with $\Gamma_{x}=\Gamma_{y}$. Let us now compare the theoretical results for the parallelepiped containers of different $\Gamma_{y} \leqslant \Gamma_{x}$ with those for the optimal parallelepiped container with $\Gamma_{x}=\Gamma_{y}$. Figure 12 shows the difference between the critical Rayleigh number for any $\Gamma_{y} \leqslant \Gamma_{x}$ and that for the optimal $\Gamma_{y}=\Gamma_{x}$. In Fig. 12(a) the difference is plotted versus the ratio $\Gamma_{y} / \Gamma_{x}, 0<\Gamma_{y} / \Gamma_{x} \leqslant 1$, for different $\Gamma_{x}=0.1$, 0.5, 1, 5, and 10. In Fig. 12(b) this difference is plotted versus $\Gamma_{x}$, for different ratios $\Gamma_{y} / \Gamma_{x}=10^{-4}$, $10^{-3}, 10^{-2}, 10^{-1}$. 
(a)

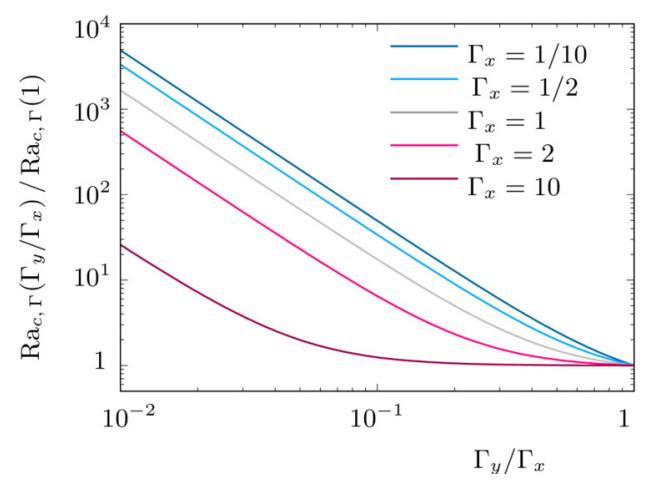

(b)

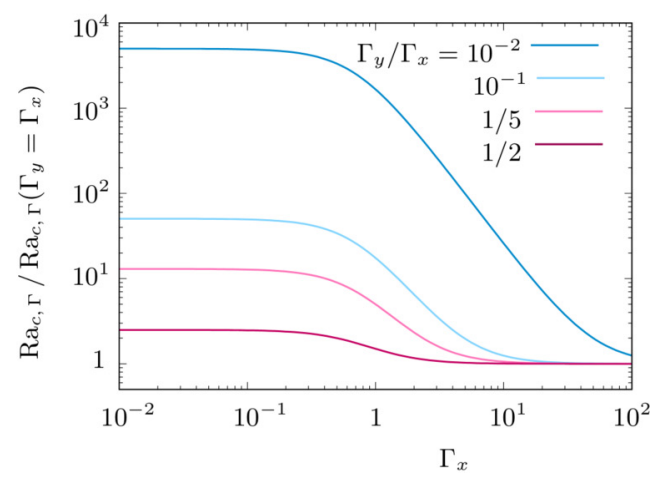

FIG. 12. (a) Ratios of the critical Rayleigh number, $\operatorname{Ra}_{c, \Gamma}\left(\Gamma_{y} / \Gamma_{x}\right)$, for the onset of convection in a parallelepiped container with different widths of the sidewalls $\left(\Gamma_{y} \leqslant \Gamma_{x}\right)$ and the critical Rayleigh number for the onset of convection in a similar container, $\operatorname{Ra}_{c, \Gamma}(1)$, for equal widths of the sidewalls $\left(\Gamma_{y}=\Gamma_{x}\right)$, as functions of $\Gamma_{y} / \Gamma_{x}$. Shown are curves for $\Gamma_{x}=0.1,0.5,1,2$, and 10. (b) Ratios of the critical Rayleigh number $\mathrm{Ra}_{c, \Gamma}$ for the onset of convection in a parallelepiped container with different widths of the sidewalls $\left(\Gamma_{y} \leqslant \Gamma_{x}\right)$ and the critical Rayleigh number for the onset of convection in a similar container with equal widths of the sidewalls $\left(\Gamma_{y}=\Gamma_{x}\right)$, as functions of $\Gamma_{x}=\Gamma$. Shown are curves for $\Gamma_{y} / \Gamma_{x}=0.01,0.1,0.2$, and 0.5 . Everywhere, the boundary conditions are no-slip for the velocity at all walls and isothermal at the plates, and adiabatic at the sidewalls for the temperature. In both panels, the calculations are according to Eq. (56).

As soon as $\Gamma_{y} \rightarrow \Gamma_{x}$, the difference between $\operatorname{Ra}_{c, \Gamma}\left(\Gamma_{y}<\Gamma_{x}\right)$ and $\operatorname{Ra}_{c, \Gamma}\left(\Gamma_{y}=\Gamma_{x}\right)$ certainly vanishes. However, for any fixed $\Gamma_{x}$, this difference grows dramatically when $\Gamma_{y} \rightarrow 0$, as one can see in Fig. 12. From Eq. (56) it follows that even when $\Gamma_{x} \rightarrow \infty$, the critical Rayleigh number grows as

$$
\operatorname{Ra}_{c, \Gamma} \approx(2 \pi)^{4}\left(1+\frac{1}{\Gamma_{x}^{2}}\right)\left(1+\frac{1}{4 \Gamma_{x}^{2}}+\frac{1}{4 \Gamma_{y}^{2}}\right) \sim \frac{4 \pi^{4}}{\Gamma_{y}^{2}} \quad \text { for } \quad \Gamma_{x} \rightarrow \infty, \quad \Gamma_{y} \rightarrow 0 .
$$

This result supports the known fact of laminarization of the confined Rayleigh-Bénard flow even for very large $\mathrm{Ra}$, as soon as the width of the three-dimensional domain becomes too small [104-107].

To conclude, we have confirmed that the optimal rectangular plates are squares, i.e., $\Gamma_{x}=\Gamma_{y}$, while for a fixed length of the domain $\Gamma_{x}$, a decreasing width of the container, $\Gamma_{y} \rightarrow 0$, leads to a growth of the critical Rayleigh number as $\sim \Gamma_{y}^{-2}$. Circular-shaped plates seem to match the optimal ones among all convex plates in Rayleigh-Bénard experiments with different sidewalls conditions, by providing the smallest critical Rayleigh number for the onset of convection among all convex plates of a given area.

\section{Optimal height of the container in Rayleigh-Bénard convection}

In this subsection, we will discuss the optimal height of the container (or, equivalently, the optimal container aspect ratio) for Rayleigh-Bénard experiments, assuming that the shape and size of the plates are prescribed. Let us recall: the optimal container height means that it provides the maximal range of the Rayleigh numbers, starting from the onset of convection, that one can achieve in Rayleigh-Bénard experiments using this container and a certain fixed Boussinesq fluid. At first glance, it might seem that choosing the highest possible container height would be the best choice, because any increase of the container height $H$ would lead to an increase of the achievable Rayleigh number that grows as cubed height of the cell $\left(\sim H^{3}\right)$. However, as we will see, this is not true.

For a fixed diameter $D$ of the heated or cooled plates, an increase of $H$ means a decrease of the container aspect ratio $\Gamma=D / H$. Thus, with increasing height of the cell, the achievable Rayleigh 
number $\mathrm{Ra}_{\max }$ grows as

$$
\operatorname{Ra}_{\max } \equiv \operatorname{Ra}_{D} \Gamma^{-3} \sim \Gamma^{-3}
$$

Here

$$
\operatorname{Ra}_{D} \equiv \frac{\alpha g \Delta_{\max } D^{3}}{\kappa \nu}
$$

is the maximal diameter-based Rayleigh number, which is determined by the maximal possible temperature difference between the plates that one can attain in the experiment, $\Delta_{\max }$, and the specific properties of the working fluid.

On the other hand, as we have already seen in the previous section, the critical Rayleigh number for the onset of convection, $\operatorname{Ra}_{c, \Gamma}$, also grows with decreasing $\Gamma$. In the limit $\Gamma \rightarrow 0, \operatorname{Ra}_{c}, \Gamma$ grows as $\sim \Gamma^{-4}$, i.e., even faster than $\mathrm{Ra}_{\max }$ ! This means that in extremely slender containers there might be no convection at all although the Rayleigh number might be huge. Thus, in a container with given shape and size of the heated and cooled plates, there is no convection if the container height is too small or too large; in both extreme situations, the achievable in the experiment Rayleigh number $\mathrm{Ra}_{\max }$ is smaller than the critical one for the onset of convection in this container, $\mathrm{Ra}_{c, \Gamma}$.

Naturally, we aim to find the optimal height, or the optimal cell aspect ratio $\Gamma$, which maximally broadens the range of the Rayleigh numbers for which convection takes place, i.e., between $\mathrm{Ra}_{c, \Gamma}$ and $\mathrm{Ra}_{\max }$. However, we want to maximize not the absolute range, $\mathrm{Ra}_{\max }-\mathrm{Ra}_{c, \Gamma}$, but the range of orders of magnitude in Rayleigh numbers. (Clearly, an experiment that exhibits Oberbeck-Boussinesq convection in a Ra range, say, from $\mathrm{Ra}_{c, \Gamma}=10^{6}$ to $\mathrm{Ra}_{\max }=10^{16}$ is much more interesting than that one from $\mathrm{Ra}_{c, \Gamma}=10^{16}$ to $\mathrm{Ra}_{\max }=2 \times 10^{16}$, although the Ra range in the latter experiment is larger. The former experiment covers 10 orders of magnitude in Rayleigh numbers, while the latter one covers much less than one order of magnitude.) This means that the aim is to maximize

$$
m \equiv \log _{10}\left(\operatorname{Ra}_{\max } / \operatorname{Ra}_{c, \Gamma}\right)=\log _{10}\left(\operatorname{Ra}_{\max }\right)-\log _{10}\left(\operatorname{Ra}_{c, \Gamma}\right) .
$$

In the following we use the notation

$$
m_{\max } \equiv \max _{\Gamma} m
$$

Let us consider convection cells with rectangular or circular plates and find the optimal $\Gamma$ that maximizes the value of $\log _{10}\left(\mathrm{Ra}_{\max } / \mathrm{Ra}_{c, \Gamma}\right.$ ) (or, equivalently, the value of $\mathrm{Ra}_{\max } / \mathrm{Ra}_{c, \Gamma}$ ). In these cases, the critical Rayleigh number for onset of convection in a container with the adiabatic sidewalls can be approximated with Eq. (101) with the corresponding constants $c_{u}$ and $c_{\theta}$ defined by Eq. (102) and Eq. (103), respectively. From Eqs. (111), (101), and (109) we obtain

$$
m=\log _{10}\left(\operatorname{Ra}_{D}\right)-\log _{10}\left[(2 \pi)^{4} \Gamma^{3}\left(1+c_{u} \Gamma^{-2}\right)\left(1+c_{\theta} \Gamma^{-2}\right)\right],
$$

which is maximal at

$$
\Gamma=\Gamma_{\mathrm{opt}} \equiv \sqrt{\frac{\sqrt{\left(c_{u}+c_{\theta}\right)^{2}+12 c_{u} c_{\theta}}-\left(c_{u}+c_{\theta}\right)}{6}} .
$$

For the optimal aspect ratio $\Gamma_{\text {opt }}$, the maximal achievable range of the Rayleigh numbers, measured in orders of magnitude, equals

$$
m_{\max }=\log _{10}\left(\operatorname{Ra}_{D}\right)-\log _{10}\left[(2 \pi)^{4} \Gamma_{\text {opt }}^{-1}\left(c_{u}+\Gamma_{\text {opt }}^{2}\right)\left(c_{\theta}+\Gamma_{\text {opt }}^{2}\right)\right] .
$$

For a parallelepiped container with adiabatic sidewalls, from Eqs. (114), (115), and (102) we obtain the following optimal aspect ratio $\Gamma_{\text {opt }}$ and maximal achievable Ra range of the orders of 
magnitude, $m_{\max }$ :

$$
\begin{gathered}
\Gamma_{\mathrm{opt}} \approx 0.478, \\
m_{\max } \approx \log _{10}\left(\mathrm{Ra}_{D}\right)-3.465 .
\end{gathered}
$$

For a cylindrical container with adiabatic sidewalls, Eqs. (114), (115), and (101) lead to

$$
\begin{gathered}
\Gamma_{\mathrm{opt}} \approx 0.456, \\
m_{\max } \approx \log _{10}\left(\operatorname{Ra}_{D}\right)-3.504 .
\end{gathered}
$$

For a cylindrical container with the conducting sidewall, $c_{u}=c_{\theta} \approx 1.4876$, and we obtain

$$
\begin{gathered}
\Gamma_{\mathrm{opt}} \approx 0.704, \\
m_{\max } \approx \log _{10}\left(\operatorname{Ra}_{D}\right)-3.940 .
\end{gathered}
$$

One can see that the optimal aspect ratio of a cylindrical or parallelepiped container with $\Gamma_{x}=\Gamma_{y}$ and adiabatic sidewalls is achieved at the aspect ratio close to $1 / 2$. The aspect ratio $\Gamma=1 / 2$ is the most popular in Rayleigh-Bénard experiments, and as we can see from our derivations, this intuitive choice is right. For conducting sidewalls, the optimal aspect ratio is slightly larger, about 0.7 , and the attainable Ra range is slightly smaller.

We can formulate the following rule of thumb [which we can call the "three and a half" rule; see Eq. (119)]: To estimate the maximal possible Ra range, measured in orders of magnitude, one needs to calculate $\mathrm{Ra}_{D}$, according to Eq. (110), which is based on the diameter of the plates, the maximal possible temperature difference between the plates that can be achieved in the experiment and the properties of the fluid used in the experiment. Subtracting 3.5 from $\log _{10}\left(\operatorname{Ra}_{D}\right)$ will give a number of orders of magnitude in Rayleigh numbers that can be attained in the optimal aspect ratio experiment.

We know now, how to estimate the optimal aspect ratio of a Rayleigh-Bénard cell, Eq. (114), and the corresponding maximal achievable range of the Rayleigh numbers in experiments or simulations, using the cell with the optimal aspect ratio, Eq. (113). Finally, let us address the question, how a deviation of the container aspect ratio from the optimal one would affect the range of the attainable Rayleigh numbers.

From the relation (113), for the attainable order of magnitude in Rayleigh numbers, $m$, we obtain

$$
\begin{array}{ll}
m \rightarrow \log _{10} \Gamma+\log _{10}\left(\frac{\operatorname{Ra}_{D}}{(2 \pi)^{4} c_{u} c_{\theta}}\right), & \Gamma \rightarrow 0, \\
m \rightarrow \log _{10} \Gamma^{-3}+\log _{10}\left(\frac{\operatorname{Ra}_{D}}{(2 \pi)^{4}}\right), & \Gamma \rightarrow \infty .
\end{array}
$$

Thus, in both limits, $\Gamma \rightarrow 0$ and $\Gamma \rightarrow \infty$, the attainable order of magnitude, $m$, decreases proportionally to $\sim \log _{10} \Gamma$. In the limit $\Gamma \rightarrow \infty$, the value of $m$ decreases three times faster than in the limit $\Gamma \rightarrow 0$. This is illustrated in Fig. 13, for the case of cylindrical containers with adiabatic sidewalls.

The intersection of the asymptotes, Eqs. (122) and (123), for, respectively, infinitesimal and infinitely large aspect ratios $\Gamma$, takes place at

$$
\Gamma=\left(c_{u} c_{\theta}\right)^{1 / 4},
$$

which for both, a parallelepiped, Eqs. (102), and a cylinder, Eqs. (103), gives $\Gamma \approx 0.84$. 


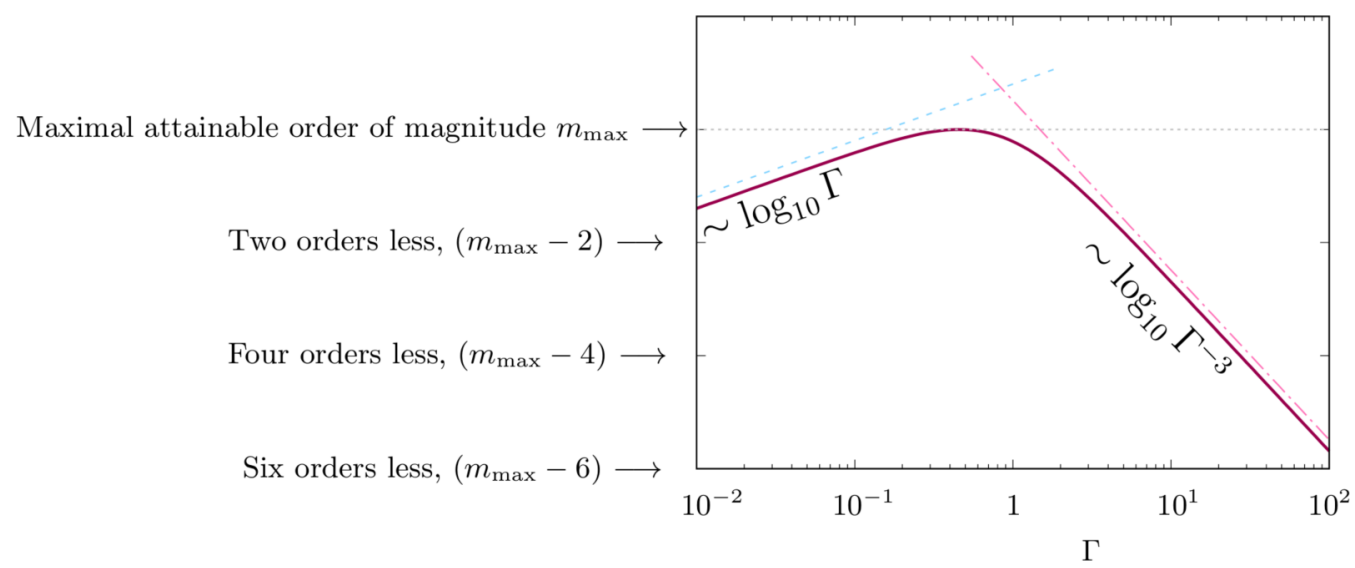

FIG. 13. The dependence of orders of magnitude in Rayleigh numbers that one can attain in RayleighBénard experiments in a cylindrical container with the adiabatic sidewall, as function of the cylinder aspect ratio $\Gamma$. The maximal value, $m_{\max }$, depends on $\mathrm{Ra}_{D}=\alpha g \Delta_{\max } D^{3} /(\kappa v)$, i.e., on the maximal Rayleigh number, which is calculated based on the diameter of the plates, $D$, the maximal possible temperature difference between the plates that one can attain in the experiment, $\Delta_{\max }$, and the specific properties of the working fluid.

Finally, we should notice that the optimal aspect ratio of the container $\Gamma$, Eq. (114), depends neither on the properties of the chosen fluid nor on the maximal temperature difference between the heated and cooled plates that can be achieved in the experiment. In other words, the optimal $\Gamma$ is independent of $\mathrm{Ra}_{D}$.

However, the maximal achievable range of the Rayleigh numbers, measured in orders of magnitude, $m_{\max }$, Eq. (115), depends on $\mathrm{Ra}_{D}$ : the larger $\mathrm{Ra}_{D}$ is, the broader $\mathrm{Ra}$ range can be achieved in the experiment. For any fixed fluid and $\Delta_{\max }$, using containers with larger sizes of the plates, one can explore a broader range (more orders of magnitude) in Rayleigh numbers. This means that not only the aspect ratio of the containers matters but also their size.

\section{RELEVANT LENGTH SCALE IN RAYLEIGH-BÉNARD CONVECTION}

In this section, we will derive that the relevant length scale in Rayleigh-Bénard convection in containers with no-slip boundaries is

$$
\ell \sim D / \sqrt{\Gamma^{2}+c_{u}}=H / \sqrt{1+c_{u} / \Gamma^{2}},
$$

where the constant $c_{u}$ is determined by the container shape and boundary conditions for the velocity. In the case of no-slip boundary conditions, $c_{u}$ follows Eq. (102) for parallelepiped domains and Eq. (103) for cylindrical domains. Thus, we will show that in the limit $\Gamma \rightarrow \infty$, the relevant length scale $\ell$ equals the height of the Rayleigh-Bénard cell, $H$, while for $\Gamma \rightarrow 0$, the length scale $\ell$ equals the cell diameter $D$ (see also [59]).

\section{A. Scaling relation for the Nusselt number close to the onset of convection and the relevant length scale $\ell$}

Combining Eqs. (17) and (32) for the state close to the onset of convection we obtain

$$
\mathrm{Ra} \gtrsim H^{4} \frac{\left\langle(\nabla \mathbf{u})^{2}\right\rangle\left\langle(\nabla \theta)^{2}\right\rangle}{\left\langle\mathbf{u}^{2}\right\rangle\left\langle\theta^{2}\right\rangle} \gtrsim H^{4} \lambda_{u} \frac{\left\langle(\nabla \theta)^{2}\right\rangle}{\left\langle\theta^{2}\right\rangle}
$$


where $\lambda_{u}$ is given by Eqs. (47) and (64) for parallelepiped and cylindrical domains, respectively. In both cases, $\lambda_{u}$ takes the form

$$
\lambda_{u}=\frac{4 \pi^{2}}{H^{2}}\left(1+c_{u} \Gamma^{-2}\right)
$$

where $c_{u}$ is given by Eqs. (102) and (103) for parallelepiped and cylindrical domains, respectively.

Applying successively Eqs. (13), (9), and (12), we derive

$$
\left\langle(\nabla \theta)^{2}\right\rangle=\left\langle\left(\nabla\left(T-T_{l}\right)\right)^{2}\right\rangle=\left\langle(\nabla T)^{2}\right\rangle-\frac{\Delta^{2}}{H^{2}}=\frac{\Delta^{2}}{H^{2}}(\mathrm{Nu}-1) .
$$

Since $\left\langle\theta^{2}\right\rangle=c \Delta^{2}$ for a certain constant $c, 0<c<1$, from this and Eqs. (126) and (128) we obtain

$$
\mathrm{Ra} \gtrsim H^{2} \lambda_{u} c^{-1}(\mathrm{Nu}-1) \text {. }
$$

[Note that the value of $c$ can be estimated with $(\mathrm{Nu}-1) / \mathrm{Nu}<(2 \pi)^{2} c \lesssim 1$. This follows from relation (129) and the fact that close to the onset $\mathrm{Nu} \sim \mathrm{Ra} / \mathrm{Ra}_{c}, \Gamma \rightarrow \infty$ for $\Gamma \rightarrow \infty$ (see, e.g., $[36,68,108])$.]

Combining Eqs. (129) and (127) we derive that near the onset of convection the Nusselt number behaves as

$$
\mathrm{Nu}-1 \sim c\left(1+c_{u} \Gamma^{-2}\right)^{-1} \mathrm{Ra},
$$

or, equivalently,

$$
f \sim\left[\frac{\mathrm{Ra}}{\left(1+c_{u} \Gamma^{-2}\right)^{3 / 2}}\right]^{2 / 3}
$$

where

$$
f \equiv(\mathrm{Nu}-1) \mathrm{Ra}^{-1 / 3} .
$$

On the other hand, in the classical turbulent regime (for not too small Pr and not extremely high $\mathrm{Ra}$ ), one expects $\mathrm{Nu}-1 \sim \mathrm{Ra}^{1 / 3}$, or, equivalently,

$$
f \sim\left[\frac{\mathrm{Ra}}{\left(1+c_{u} \Gamma^{-2}\right)^{3 / 2}}\right]^{0} .
$$

From Eqs. (131) and (133) we see that the respective scaling quantity is not the Rayleigh number based on the height of the cell, $H$, but the Rayleigh number $\mathrm{Ra}_{\ell}$,

$$
\mathrm{Ra}_{\ell} \equiv \operatorname{Ra}\left(1+c_{u} \Gamma^{-2}\right)^{-3 / 2}
$$

This means that for the relevant length scale $\ell$ in Rayleigh-Bénard convection it holds that

$$
\ell^{3} \sim \frac{H^{3}}{\left(1+c_{u} \Gamma^{-2}\right)^{3 / 2}},
$$

which is equivalent to relation (125). Thus, for large $\Gamma$, the length scale $\ell$ is rather $H$, while for very small $\Gamma$, it is rather $D$.

Finally, we remark about the prefactors in the scalings of the dimensional heat transport $\mathrm{Nu}$ versus Ra near the onset of convection, for small $\Gamma$. As we can see from Eq. (130), the prefactor is smaller for smaller $\Gamma$ in the scaling $\mathrm{Nu}$ versus Ra. However, in the relation $\mathrm{Nu}$ versus the Rayleigh number normalized with the Rayleigh number for the onset of convection, i.e., $\mathrm{Ra} / \mathrm{Ra}_{c}, \Gamma$, the prefactor is larger for smaller $\Gamma$. More precisely,

$$
\mathrm{Nu}-1 \sim c\left(1+\frac{c_{\theta}}{\Gamma^{2}}\right) \frac{\mathrm{Ra}}{\mathrm{Ra}_{c, \Gamma}}
$$


Thus, in a plot of the heat transport scaling with $\mathrm{Ra} / \mathrm{Ra}_{c, \Gamma}$ near the onset of convection, one would expect a steeper growth of $\mathrm{Nu}$ for slender containers with small $\Gamma$, although convection in such containers starts later, i.e., for larger values of $\mathrm{Ra}_{c, \Gamma}$.

\section{B. Comparison with experiments and numerical simulations}

Based on the results of (almost) Oberbeck-Boussinesq experiments and direct numerical simulations of Rayleigh-Bénard convection in cylindrical containers [59,87,89,107,109-139], let us now illustrate that the derived relevant length scale in Rayleigh-Bénard convection is $\ell$, Eq. (125), and the corresponding Rayleigh number is $\mathrm{Ra}_{\ell}$, Eq. (134). For cylindrical Rayleigh-Bénard convection cells, $c_{u}$ is defined by Eq. (103), which in combination with Eq. (135) gives the relevant scaling quantity

$$
\mathrm{Ra}_{\ell} \approx \operatorname{Ra}\left(1+1.49 \Gamma^{-2}\right)^{-3 / 2}
$$

In Fig. 14 we present the results [59,87,89,107,109-139] for fluids with Prandtl numbers ranging from 0.7 to about 6 , which correspond to the most popular fluids of air and water at room temperature. The range of the considered aspect ratios $\Gamma$ of the cylindrical Rayleigh-Bénard cell used in these experiments is very broad, from $1 / 32$ to 32 .

Figure 14(a) shows the dependence of the compensated Nusselt number, $(\mathrm{Nu}-1) / \mathrm{Ra}^{1 / 3}$, on the Rayleigh number Ra. A pronounced shape of this dependence is determined by the data for $\Gamma=1$ and $\Gamma=1 / 2$, which are the most popular in experiments and simulations. However, the data for extremely small $\Gamma$ lie completely off the main trend; see the data points for $\operatorname{Pr}=4.38$, for $\Gamma$ from $1 / 32$ to $1 / 5$ from [109]. For any fixed Ra they simply show that $\mathrm{Nu}$ is larger for smaller $\Gamma$.

Another conspicuous feature of the plot Fig. 14(a) is related to the measurements for extremely large $\mathrm{Ra}$, where at high Rayleigh numbers one observes a transition, associated with an increased exponent $(>1 / 3)$ in the $\mathrm{Nu}$ versus Ra scaling. The Göttingen group (see $[122,126-128,134,140]$ ) studied the transition to the ultimate regime $\mathrm{Ra}_{u, \Gamma}$ in Rayleigh-Bénard convection [141-143], for the cylindrical containers of the aspect ratios $\Gamma=1,1 / 2$ and $1 / 3$. They found that the onset occurs at $\mathrm{Ra}$ about $10^{14}$, which is consistent with the theoretical estimate by Grossmann and Lohse [143]; however, it shows a certain $\Gamma$-dependence. Their reported empirical formula is $\operatorname{Ra}_{u, \Gamma} \propto \Gamma^{-3.04}$ [144]. Furthermore, based on different Oberbeck-Boussinesq and non-Oberbeck-Boussinesq experimental data for the aspect ratios of the cylindrical containers $0.23 \leqslant \Gamma \leqslant 1.14$ [126-128,134,145-150], Roche $[147,151]$ also proposed that for small $\Gamma$, the Rayleigh number for the onset of the transition to the ultimate regime changes approximately as $\operatorname{Ra}_{u, \Gamma} \sim \Gamma^{-3}$.

In Fig. 14(a) the transition, which was observed in the Göttingen almost Oberbeck-Boussinesq measurements $[122,126-128,134,140]$ for the cylindrical containers of the aspect ratios $\Gamma=1,1 / 2$, and $1 / 3$, is marked with vertical lines. However, in accordance with our theory, in the rescaled plot [see Fig. 14(b)], where the reduced Nusselt number is plotted versus $\mathrm{Ra}_{\ell}$, these vertical lines collapse at the same value

$$
\operatorname{Ra}_{\ell, u} \approx 2.4 \times 10^{13}
$$

Substituting $\mathrm{Ra}_{\ell}=\mathrm{Ra}_{\ell, u}$ from Eq. (138) into Eq. (137) and evaluating Ra (which we denote as $\left.\mathrm{Ra}_{u, \Gamma}\right)$, we obtain the expected $\Gamma$-scaling of the transition

$$
\operatorname{Ra}_{u, \Gamma} \approx \operatorname{Ra}_{\ell, u}\left(1+1.49 \Gamma^{-2}\right)^{3 / 2} .
$$

In the limit $\Gamma \rightarrow \infty$, the value of $\operatorname{Ra}_{u, \Gamma}$ approaches $\operatorname{Ra}_{\ell, u}$, i.e., $\operatorname{Ra}_{u, \Gamma} \rightarrow \operatorname{Ra}_{\ell, u}$. In the other limit, $\Gamma \rightarrow 0$, according to the theory, the critical Rayleigh number for the transition should scale as

$$
\operatorname{Ra}_{u, \Gamma} \sim \Gamma^{-3}
$$

which is in full agreement with the Göttingen experimental data [122,126-128,134,140,144] as well as with analysis of further measurement data [145-150], conducted by Roche [147,151]. 
(a)

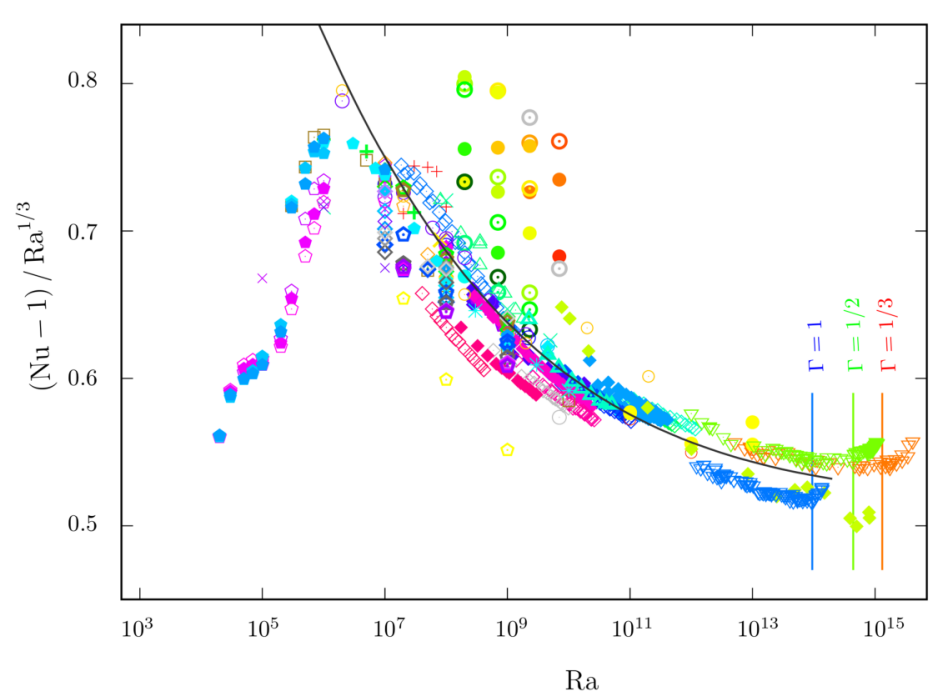

(b)

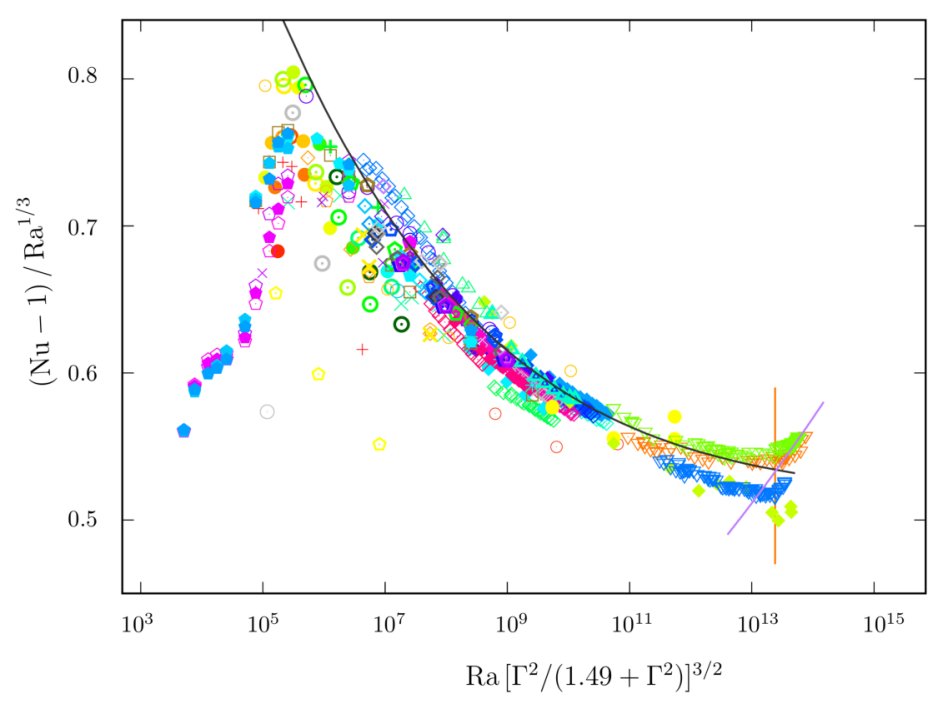

$\Gamma=1 / 32$ (cylinder), $\operatorname{Pr}=4.38[109]$ $\Gamma=1 / 28$ (cylinder) $\mathrm{Pr}=4.38 \quad 109$ - $\Gamma=1 / 24$ (cylinder), $\operatorname{Pr}=4.38$ 109 $\Gamma=1 / 20$ (cylinder), $\mathrm{Pr}=4.38$ $\Gamma=1 / 20$ (cylinder), $\mathrm{Pr}=4.38$
$\Gamma=1 / 18$ (cylinder), $\mathrm{Pr}=4.38 \quad 109$ $\Gamma=1 / 18$ (cylinder), $\operatorname{Pr}=4.38109$
$\Gamma=1 / 16$ (cylinder), $\operatorname{Pr}=4.38 \quad 109$ $\Gamma=1 / 16$ (cylinder), $\operatorname{Pr}=4.38109$
$\Gamma=1 / 14$ (cylinder) $\operatorname{Pr}=4.38 \quad 109$ $\Gamma=1 / 12$ (cylinder), $\operatorname{Pr}=4.38 \quad 109$

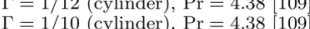
$\Gamma=1 / 10$ (cylinder), $\operatorname{Pr}=4.38[109]$.
$\Gamma=1 / 8$ cylinder $), \operatorname{Pr}=4.38[109]$ $\Gamma=1 / 8$ (cylinder), $\operatorname{Pr}=4.38[109]$ $\Gamma=1 / 7$ (cylinder), $\operatorname{Pr}=4.38[109$
$\Gamma=1 / 6$ (cylinder) $\operatorname{Pr}=4.38$ $\Gamma=1 / 6$ (cylinder), $\mathrm{Pr}=4.38$ (109

P $\mathrm{T}=1 / 5$ (cylinder), $\mathrm{Pr}=4.38109$ $=1 / 4$ (cylinder), $\operatorname{Pr}=4.38[109]$

$\Gamma=0.275$ (cylinder), $\operatorname{Pr} \approx 4.4[110]$

$\Gamma=1 / 3$ (cylinder), $\operatorname{Pr}=4.38[109]$
$\Gamma=1 / 3,1 / 2,1$ (cylinder), $\operatorname{Pr}=4.38[59]$

$\Gamma=1 / 3,1 / 2,1$ (cylinder), $\operatorname{Pr}=4.38$
$\Gamma=0.427$ (cylinder), $\operatorname{Pr} \approx 4.4[110]$

$\Gamma=1 / 2$ (cylinder), $\operatorname{Pr}=4.38[109]$

$\Gamma=1 / 2$ (cylinder), $\operatorname{Pr} \approx 4.38$
$\Gamma=1 / 2$ (cylinder), $\operatorname{Pr}=4.38[112,113]$

$\Gamma=1 / 2$ (cylinder), $\operatorname{Pr}=4.38[112,113]$
$\Gamma=0.667$ (cylinder), $\operatorname{Pr} \approx 4.4[110]$

$\Gamma=0.967$ (cylinder), $\operatorname{Pr} \approx 4.38[114]$

$\Gamma=0.967$ (cylinder), $\mathrm{Pr} \approx 4.38[114]$

$\Gamma=0.981$ (cylinder), $\mathrm{Pr} \approx 4.4[110]$

$\Gamma=0.982$ (cylinder), $\operatorname{Pr} \approx 4.38[11$

$\Gamma=1$ (cylinder), $\operatorname{Pr}=4.38[112$

$\Gamma=1$ (cylinder), $\operatorname{Pr}=4.38[115]$
$\Gamma=1$ (cylinder), $4.38 \leq \operatorname{Pr} \leq 6.41[116]$

$\Gamma=1$ (cylinder), $4.38 \leq \operatorname{Pr} \leq 6.4$
$\Gamma=1$

$\Gamma=1$ (cylinder), $\operatorname{Pr}=5.2[117]$
$\Gamma=1$ (cylinder), $\operatorname{Pr}=4.38[118]$

$\Gamma=1$ (cylinder), $\operatorname{Pr}=4.38[118]$
$\Gamma=1$ (cylinder), $\operatorname{Pr}=2[119]$

$\Gamma=1$ (cylinder), $\mathrm{Pr}=2[119$

$\Gamma=1$ (cylinder), $\operatorname{Pr}=3[119$

$\Gamma=1$ (cylinder), $\operatorname{Pr}=5[119$

$\Gamma=1.003$ (cylinder), $\operatorname{Pr} \approx 4.38[114$

$\Gamma=1.506$ (cylinder), $\operatorname{Pr} \approx 4.38[114$

$\Gamma=2.006$ (cylinder), $\operatorname{Pr} \approx 4.38$ [11

$\Gamma=3.010$ (cylinder), $\operatorname{Pr} \approx 4.38[114$

$\Gamma=6.020$ (cylinder), $\operatorname{Pr} \approx 4.38[114$

$\Gamma=1 / 5$ (cylinder), $\operatorname{Pr}=1[107]$

$\Gamma=0.23$ (cylinder), $\operatorname{Pr}=0.7[120$

$\Gamma=1 / 4$ (periodic), $\operatorname{Pr}=1[121]$

$\Gamma=1 / 3$ (cylinder), $\operatorname{Pr} \approx 0.8[122]$

$\Gamma=1 / 2$ (periodic), $\mathrm{Pr}=1[121]$

$\Gamma=1 / 2$ (cylinder), $\operatorname{Pr}=0.7[123$

$\Gamma=1 / 2$ (cylinder), $\operatorname{Pr}=0.7[124]$

$\Gamma=1 / 2$ (cylinder), $\operatorname{Pr}=0.8[87,89]$

$\Gamma=1 / 2$ (cylinder), $\operatorname{Pr}=0.7[120]$

$\Gamma=1 / 2$ (cylinder), $\operatorname{Pr} \approx 0.86[125]$

$\Gamma=1 / 2$ (cylinder), $\operatorname{Pr} \approx 0.8[126-128]$

$\Gamma=3 / 4$ (periodic), $\operatorname{Pr}=1[121]$

$\Gamma=1$ (periodic), $\mathrm{Pr}=1[121]$

$\Gamma=1$ (cylinder), $\operatorname{Pr}=0.7[123]$

$\Gamma=1$ (cylinder), $\operatorname{Pr}=1[129]$

$\Gamma=1$ (cylinder), $\operatorname{Pr}=0.7[130]$

$\Gamma=1$ (cylinder), $\operatorname{Pr} \approx 0.74[59,131]$

$\Gamma=1$ (cylinder), $\operatorname{Pr} \approx 0.84[59,131]$

$\Gamma=1$ (cylinder), $\operatorname{Pr}=1[132$

$\Gamma=1$ (cylinder), $\operatorname{Pr}=0.786[133]$

$\Gamma=1$ (cylinder), $\operatorname{Pr}=0.7[119]$

$\Gamma=1$ (cylinder), $\operatorname{Pr}=1[119$

$\Gamma=1$ (cylinder), $\operatorname{Pr} \approx 0.8[134]$

$\Gamma=1.25$ (cylinder), $\operatorname{Pr}=0.7[123]$

$\Gamma=1.5$ (cylinder). $\mathrm{Pr}=0.7[123]$

$\Gamma=1.75$ (cylinder), $\mathrm{Pr}=0.7[123$

$\Gamma=2$ (cylinder), $\operatorname{Pr}=0.7[123]$

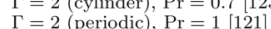

$\Gamma=2.25$ (cylinder), $\operatorname{Pr}=0.7[123]$

$\Gamma=2.5$ (cylinder), $\mathrm{Pr}=0.7$ [123]

$\Gamma=2.75$ (cylinder), $\operatorname{Pr}=0.7[123$

$\Gamma=3$ (cylinder), $\operatorname{Pr}=0.7[123$

$\Gamma=3$ (periodic), $\mathrm{Pr}=1[121]$

$\Gamma=4$ (cylinder), $\mathrm{Pr}=0.7[123]$

$\Gamma=4$ (periodic), $\mathrm{Pr}=1[121]$

$\Gamma=5$ (cylinder), $\operatorname{Pr}=0.7[135]$

$\Gamma=6$ (cylinder), $\mathrm{Pr}=0.7[123$

$\Gamma=8$ (periodic), $\mathrm{Pr}=1[121]$

$\Gamma=10$ (cylinder), $\mathrm{Pr}=0.7[136]$

$\Gamma=16$ (periodic), $\mathrm{Pr}=1[121$

(periodic), $\mathrm{Pr}=1[121]$

$\Gamma=1, \mathrm{Pr}=1$, theo
slope $\mathrm{Nu} \sim \mathrm{Ra}^{0.38}$

FIG. 14. (a) Compensated $\mathrm{Nu}$ vs $\mathrm{Ra}$, as obtained in (almost) Oberbeck-Boussinesq experiments and numerical simulations of Rayleigh-Bénard convection in a cylinder or in a three-dimensional cell with periodic boundary conditions, for Prandtl numbers $\operatorname{Pr}$ that correspond to water $(\operatorname{Pr} \approx 4)$ and air $(\operatorname{Pr} \approx 1)$, for different $\Gamma[59,87,89,107,109-139]$. Most data are for $\Gamma=1$ and $1 / 2$, which form the shape of this dependence. The data for extremely small $\Gamma(\operatorname{Pr}=4.38)$ show no discernible dependence. Vertical lines indicate the onset of the transition at high Ra, observed in Göttingen experiments [126-128] (the onset moves to higher Ra with decreasing $\Gamma$ ). (b) Compensated $\mathrm{Nu}$ vs Ra based on the proper length scale $\ell$, for the same data as in (a). Now the data for extremely small $\Gamma$ follow the general trend and the transition happens at the same location for all $\Gamma$ (the vertical lines from (a) merge into one line). The legend on the right corresponds to both panels, (a) and (b). Figure adapted from [59]. 
Finally, we notice that the data by Hartmann et al. [109] for extremely small $\Gamma$, from $1 / 32$ to $1 / 5$, for $\operatorname{Pr}=4.38$, which were completely off from the main trend and seemed to scatter when plotted versus Ra [as in Fig. 14(a)], start to follow the trend, if they are plotted versus Ra $\mathrm{a}_{\ell}$; see Fig. 14(b). In Fig. 14(b) the data for different $\Gamma$, from 1/32 to 32, show similar behavior and collapse on the theoretical curve of the Grossmann and Lohse predictions [137-139]. This again supports the fact that the relevant length scale in Rayleigh-Bénard convection is $\ell$, Eq. (125), and the corresponding relevant Rayleigh number is $\mathrm{Ra}_{\ell}$, Eq. (137).

\section{FURTHER RELATIONS IN THERMALLY DRIVEN FLOWS THAT FOLLOW FROM THE POINCARÉ-FRIEDRICHS INEQUALITIES AND ANALYSIS OF THE LEADING LAPLACE EIGENMODES}

Relations which we have derived in this paper and which are closely related to the PoincaréFriedrichs inequalities, can be useful in various other applications. In the next subsection, we will show, for example, how one can estimate the upper bound for the Reynolds number through the combination of the Rayleigh, Nusselt, and Prandtl numbers and the aspect ratio of the container. This estimate is accurate near the onset of convection but formally also holds in the limit $\mathrm{Ra} \rightarrow \infty$. In the following subsection, we will give another example and show how one can estimate the range of the convection roll size in multiple-state turbulent two-dimensional Rayleigh-Bénard convection. In the last subsection, we will briefly discuss rotating Rayleigh-Bénard convection.

\section{A. Relation between kinetic energy and its dissipation}

Let us consider, as previously, the case when the velocity satisfies Dirichlet (no-slip) boundary conditions at all boundaries. Applying subsequently Eqs. (11), (8), and (32), we obtain

$$
\frac{v^{3}}{H^{4}}(\mathrm{Nu}-1) \frac{\mathrm{Ra}}{\operatorname{Pr}^{2}}=\epsilon_{u} \equiv v\left\langle(\nabla \mathbf{u})^{2}\right\rangle \geqslant v \lambda_{u}\left\langle\mathbf{u}^{2}\right\rangle .
$$

For a rectangular two-dimensional domain or a parallelepiped three-dimensional domain, using Eq. (47), from Eq. (141) we derive that as soon as the Rayleigh number exceeds the critical Rayleigh number for the onset of convection, the fluid motion starts and the following relation holds:

$$
\frac{\nu^{3}}{H^{4}}(\mathrm{Nu}-1) \frac{\mathrm{Ra}}{\operatorname{Pr}^{2}} \geqslant v \frac{4 \pi^{2}}{H^{2}}\left(1+\frac{1}{\Gamma^{2}}\right)\left\langle\mathbf{u}^{2}\right\rangle .
$$

Introducing the Reynolds number Re, which is based on the total kinetic energy in the system,

$$
\operatorname{Re} \equiv\left\langle\mathbf{u}^{2}\right\rangle^{1 / 2} H / v
$$

from Eq. (142) we obtain the following inequality:

$$
(\mathrm{Nu}-1) \frac{\mathrm{Ra}}{\operatorname{Pr}^{2}} \geqslant 4 \pi^{2}\left(1+\frac{1}{\Gamma^{2}}\right) \operatorname{Re}^{2},
$$

which relates the kinetic energy with the dissipation rate or, in other words, the Reynolds number with $\mathrm{Ra}, \mathrm{Nu}, \mathrm{Pr}$, and $\Gamma$. Relation (144) can be reformulated in a simper form if we introduce the quantity $B$,

$$
B \equiv \frac{\operatorname{Re}^{2} \operatorname{Pr}^{2}}{\mathrm{Ra}(\mathrm{Nu}-1)} .
$$

Thus, using relation (144) we conclude that as soon as a convective fluid motion sets in, for the integral quantity $B$, Eq. (145), we will find to hold

$$
B \leqslant\left[4 \pi^{2}\left(1+\Gamma^{-2}\right)\right]^{-1} .
$$


(a)

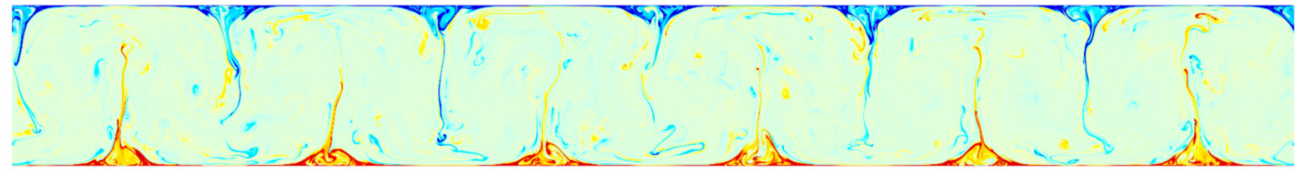

(b)

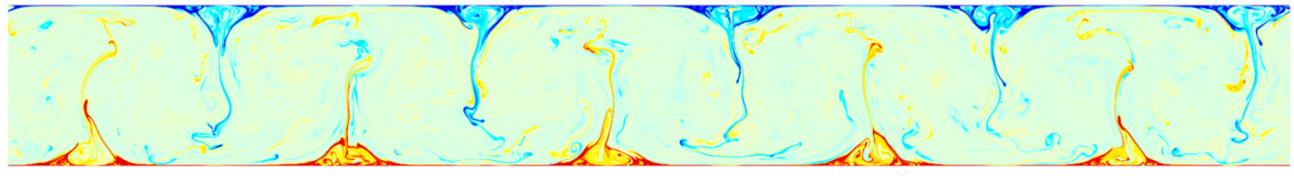

(c)

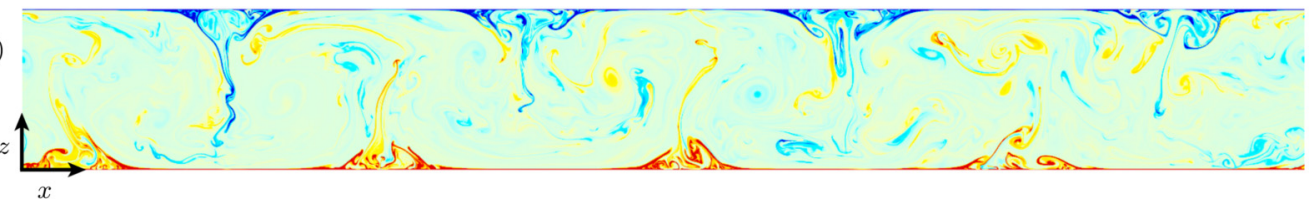

FIG. 15. Snapshots of the temperature for the three statistically stable turbulent states in two-dimensional turbulent Rayleigh-Bénard convection with no-slip boundary conditions at the top and bottom plates and periodic boundary conditions at the sides, for $\mathrm{Ra}=10^{10}, \mathrm{Pr}=10, \Gamma=8$ : (a) $\Gamma_{r}=2 / 3(m=12, \mathrm{Nu} \approx 105.2)$, (b) $\Gamma_{r}=4 / 5(m=10, \mathrm{Nu} \approx 101.5)$, and (c) $\Gamma_{r}=1(m=8, \mathrm{Nu} \approx 96.8)$. One can see that for exactly the same control parameters $\operatorname{Ra}, \operatorname{Pr}$ and $\Gamma$, the system can have different statistically steady states, with different number of rolls and different heat transport properties. Adopted from [153].

This relation gives an upper bound for the quantity $B$. It is also useful for the derivation of the range of the possible sizes of the convection rolls in two-dimensional turbulent Rayleigh-Bénard convection, as we will see in the next subsection.

\section{B. Multiple states in two-dimensional turbulent Rayleigh-Bénard convection}

Another example of the applicability of our theoretical approach is related to the wall-bounded flows that for the same control parameters can take different statistically stationary turbulent states. Here, again, we consider two-dimensional turbulent Rayleigh-Bénard convection. In wide containers with no-slip walls, these convective flows can take different statistically stationary turbulent states, which are characterized by different transport properties reflected in different values of the Nusselt and Reynolds numbers, even for exactly the same values of the control parameters, i.e., the Rayleigh and Prandtl numbers (see [152-155]).

Depending on the initial flow conditions, the convective system with specified control parameters $\mathrm{Ra}, \mathrm{Pr}$, and $\Gamma$, can end up in different states. The state of the system is characterized by the number $m$ of convection rolls that develop in a wide container with the aspect ratio $\Gamma$. The mean aspect ratio of the convection rolls is then equal to

$$
\Gamma_{r}=\Gamma / m \text {. }
$$

The more efficient heat transport is provided by slender rolls, i.e., for the smaller $\Gamma_{r}[152,153]$; see Fig. 15. But in general, what values can $\Gamma_{r}$ take? Here we answer this question, generalizing the approach proposed in Wang et al. [153].

We assume that the two-dimensional convective flow is just a set of elliptical rolls of similar shape, which are attached to each other in the horizontal direction $x$. Since all rolls are similar, let us consider a single roll which is described by a streamfunction $\Psi$,

$$
\Psi(x, z)=(\xi+\eta) \frac{z^{2}}{2}+(\xi-\eta) \frac{x^{2}}{2}
$$


in the area

$$
\Omega \equiv\left[-\Gamma_{r} H / 2, \Gamma_{r} H / 2\right] \times[-H / 2, H / 2],
$$

where $\xi \geqslant \eta \geqslant 0$. Here $\eta$ is the strain and $\xi$ is half of the vorticity; the balance between them determines the elliptical shape of the convection roll. More precisely, the aspect ratio of the roll, $\Gamma_{r}$, is related to the strain $\eta$ and vorticity $\xi$ through the relation

$$
\Gamma_{r}=\sqrt{(\xi+\eta) /(\xi-\eta)}
$$

or, in other words,

$$
\frac{\eta}{\xi}=\frac{\Gamma_{r}^{2}-1}{\Gamma_{r}^{2}+1} .
$$

Now we extract the horizontal and vertical components of the velocity $\mathbf{u} \equiv\left(u_{x}, u_{z}\right)$ from the streamfunction $\Psi$, which are, respectively, $u_{x}(x, z)=\partial \Psi / \partial z$ and $u_{z}(x, z)=-\partial \Psi / \partial x$, and evaluate the Reynolds number of the roll,

$$
\operatorname{Re}_{r} \equiv\left\langle(\partial \Psi / \partial x)^{2}+(\partial \Psi / \partial z)^{2}\right\rangle^{1 / 2} H / \nu,
$$

where the average is made over $\Omega$. With that we estimate the Reynolds number as follows:

$$
\operatorname{Re}^{2} \geqslant \operatorname{Re}_{r}^{2}=\frac{\xi(\xi+\eta) H^{4}}{6 v^{2}}
$$

According to Eq. (150), in order to form a roll, the strain must be smaller than the vorticity, $\eta<\xi$, which together with Eq. (153) gives

$$
\operatorname{Re}^{2}>\frac{\eta^{2} H^{4}}{3 v^{2}}
$$

On the other hand, there is the exact global balance (see, e.g., [156]) of the total enstrophy $\omega^{2}$ and the mean kinetic energy dissipation rate $\epsilon_{u}$, namely,

$$
4 v \xi^{2}=v \omega^{2}=\epsilon_{u} .
$$

Combining Eqs. (155), (11), and (145), we obtain

$$
\xi^{2}=\frac{v^{2}}{4 H^{4}} \frac{\mathrm{Re}^{2}}{B} .
$$

Applying successively Eqs. (151) and (156) to the relation (154), we derive

$$
\operatorname{Re}^{2}>\left(\frac{\Gamma_{r}^{2}-1}{\Gamma_{r}^{2}+1}\right)^{2} \frac{\mathrm{Re}^{2}}{12 B},
$$

which is equivalent to the following lower bound for the quantity $B$, Eq. (145),

$$
B>\frac{1}{12}\left(\frac{\Gamma_{r}^{2}-1}{\Gamma_{r}^{2}+1}\right)^{2} .
$$

The upper bound for the integral quantity $B$ was actually derived in the previous subsection. As soon as convection maintained by the considered roll of the aspect ratio $\Gamma_{r}$ is able to sustain (and does not decay), the relation (146) should hold also for $\Gamma_{r}$ taken instead of $\Gamma$. Thus, we obtain

$$
B \leqslant \frac{1}{4 \pi^{2}\left(1+\Gamma_{r}^{-2}\right)} .
$$


Combining (158) and (159) we finally derive a constraint for possible values of the convection roll aspect ratio $\Gamma_{r}$ in the case of no-slip plates:

$$
\pi^{2}\left(1+\frac{1}{\Gamma_{r}^{2}}\right) \leqslant 3\left(\frac{\Gamma_{r}^{2}+1}{\Gamma_{r}^{2}-1}\right)^{2},
$$

which gives a principle range of possible $\Gamma_{r}$ :

$$
0.7 \lesssim \Gamma_{r} \lesssim 1.6
$$

This estimate is very precise as one can conclude from comparison with plenty numerical simulations reported in Wang et al. [153]. Which exactly states are realizable, depends on particular values of $B$, i.e., on particular combinations of $\mathrm{Ra}, \mathrm{Pr}, \mathrm{Nu}$, and $\mathrm{Re}$ (see [153]).

In the three-dimensional case, the typical size of the rolls can differ from those in the above discussed two-dimensional case, because the corresponding leading eigenmodes of the Laplace operator are different. All this needs further investigations, to understand better the formation and dynamics of the superstructures in turbulent Rayleigh-Bénard convection in wide domains (see, e.g., [157-159]).

\section{The problem of the whispering gallery and rotating Rayleigh-Bénard convection}

Another example is again closely related to the studies by Lord Rayleigh. Six years before Lord Rayleigh published his seminal "attempt to examine how far the interesting results obtained by Bénard in his careful and skilful experiments can be explained theoretically" [5], he explained an interesting acoustical phenomenon known as the problem of the whispering gallery in Saint Paul's Cathedral in London [160]: a high-frequency whisper or bird call can propagate along the curved wall. So if the whispering person is standing near the wall, he or she can be heard by someone who is far from him but is also standing near the wall. Lord Rayleigh wrote that the phenomena "indicate that sonorous vibrations have a tendency to cling to concave surface" [160] and explained it by considering the eigenmodes of the Laplace operator in a circular domain. The eigenfunctions $\sim J_{n}\left(2 j_{n k} r / D\right) \cos (n \phi)$ with the corresponding eigenvalues $\lambda=4 j_{n k}^{2} / D^{2}$, for the first nontrivial root, $j_{n k}$, of the Bessel function $J_{n}$ for $k=1$ and sufficiently large $n$ are the supporters of the whispering gallery phenomena. [One can see an illustration of such eigenfunctions in Fig. 9(f) for $k=1$ and not that large but representative $n=25$.] It was shown that for larger eigenvalues the associated eigenfunctions are localized closer to the sidewall.

In fact, the problem of the whispering gallery is closely related to the problem of rapidly rotating Rayleigh-Bénard convection, where the cylindrical sample is rotated very fast around its vertically aligned axis so that convection is suppressed in the core part of the domain and the fluid motion takes place close to the sidewall only. This phenomenon is known as wall modes in rotating RayleighBénard convection (see [78-84,161]).

The wall modes, that occur at the onset of convection prior to the bulk modes, are very important as they persist (in different forms) through all regimes of rotating Rayleigh-Bénard convection, where rotation dominates or at least influences processes inside the convection cell [162]. The wall modes are connected to the recently discovered boundary zonal flows in turbulent regime of rotating Rayleigh-Bénard convection (see $[87,89,125,162,163]$ ), which first were observed in cylindrical containers of small aspect ratio, for $\Gamma \leqslant 1 / 2$ [87,163], and later also for $\Gamma \geqslant 1$ (see $[89,162]$ ). Thus, with increasing thermal driving (or with decreasing rotation rate) the linear wall modes start to interact with each other and also with the occurring bulk modes and then finally form the boundary zonal flow, which remains till buoyancy start to strongly dominate over rotation (or, in other words, the role of rotation becomes negligible).

The wall modes are extremely robust, as it was demonstrated in a recent study by Favier and Knobloch [86,88]. They seem to stubbornly drift along any wall and follow its contour, independently of the presence of geometric obstacles. Favier and Knobloch [86] provided the evidence of the robustness of the wall modes: the introduction of an extreme radial barrier, which extends from 
the cylinder centerline to its sidewall through the whole height of the cylinder, had almost no effect on the wall mode properties, compared to the case without the barrier. It seems that the wall modes do not require the shape of the sidewall to be concave (from inside)! (With this respect one might wonder, whether it is possible, being inside Saint Paul's Cathedral near the wall, to hear a whisper of a person standing at a distance, but also inside and near the wall, if a huge soundproof screen would be placed from the center of the Cathedral to its wall.)

In light of the here presented study, it might be reasonable to revisit the problem of the stability and properties of the wall modes close to their onset, in terms of the eigenmodes of the Laplace operator in cylindrical domains of different aspect ratios, with Neumann boundary conditions. For such analysis, one needs the roots of the derivative of the Bessel function $J_{n}$, i.e., $\widetilde{j}_{n k}$, for a certain sufficiently large number of $n$, which nowadays are well studied. Let us recall that in a numerical linear stability analysis of rotational Rayleigh-Bénard convection in a cylindrical sample, conducted by Goldstein et al. [81], two types of modes were found: the "fast modes," which locate near the cylinder sidewall and which have relatively large precession velocity, and the "slow modes," which show their largest values in the central part of the domain. These modes resemble the eigenfunctions of the Laplace operator in a circular domain, $\sim J_{n}\left(2 j_{n k} r / D\right) \cos (n \phi)$, with $k=1$ and large $n$ in the case of fast modes (see Fig. 2 in [81]) and with $k \gg 1$ and $n \lesssim k$ in the case of slow modes (see Fig. 7 in [81]). The fast modes are naturally related to the here discussed wall modes, trapped modes, and Rayleigh's whispering gallery modes.

With respect to the geostrophic regime in rotating Rayleigh-Bénard convection, recent studies $[87,89,125,162-164]$ showed that the heat transport in the systems with strong thermal driving (i.e., at very high Rayleigh numbers) and under rapid rotation (at very small Ekman numbers) is highly influenced by the boundary zonal flows, which, however, should be of little relevance in astrophysical systems. With that a question occurs [88], how should rotating Rayleigh-Bénard experiments be designed, to gain the relevant knowledge on geo- and astrophysical flows in the laboratory? How to disentangle the contributions and roles of the bulk and boundary zonal flows to all processes inside the convection cell, including the dynamics and global heat and momentum transport? What is the optimal shape of the container to study rotating Rayleigh-Bénard convection in the geostrophic regime? These problems and many others related to the shape of the container in rotating Rayleigh-Bénard convection certainly need further investigations.

\section{CONCLUSIONS AND OUTLOOK}

Investigation of turbulent Rayleigh-Bénard convection for very large Rayleigh numbers numerically or experimentally is challenging. To save resources, one is tempted to perform measurements or simulations at possibly small aspect ratio $\Gamma$ of the Rayleigh-Bénard convection cell. In this paper, we have studied how $\Gamma$, especially small $\Gamma$, influences processes inside the convection cell and how it affects the different flow regimes, from the onset of convection and up to the transition to the ultimate regime.

We have proved analytically that the critical Rayleigh number $\mathrm{Ra}_{c, \Gamma}$ for the onset of convection in containers of different shapes, with no-slip boundaries, for small $\Gamma$ grows as $\sim\left(1+c_{u} \Gamma^{-2}\right)(1+$ $\left.c_{\theta} \Gamma^{-2}\right), c_{u}>0, c_{\theta}>0$, Eqs. (29), (33), and (34). In order to derive accurate estimates of $\mathrm{Ra}_{c, \Gamma}$, we assumed that in the expansions of the temperature and velocity by the onset of convection in terms of the eigenfunctions of the Laplace operator, the contributions of the constant-sign eigenfunctions, both in the horizontal and vertical directions, vanish. With that we have derived $\operatorname{Ra}_{c, \Gamma} \approx$ $(2 \pi)^{4}\left(1+c_{u} \Gamma^{-2}\right)\left(1+c_{\theta} \Gamma^{-2}\right)$, where the constants $c_{u}$ and $c_{\theta}$ are determined by the container shape and boundary conditions for the velocity and temperature, respectively; see Eqs. (54)-(56), (67)-(68), and (101)-(103). For the regime close to the onset of convection, we also derived some relations for the Nusselt number, Eq. (130), and Reynolds number, Eq. (144). In the derivations we assumed the validity of the Navier-Stokes equations under Oberbeck-Boussinesq approximation, which in real situations of extremely small aspect ratios $\Gamma \ll 1$ would not hold; they would fail in the case of extremely tall containers as well as in the case of extremely thin containers. 
We demonstrated that the derived estimates for the critical Rayleigh number for the onset of convection are in very good agreement with the numerically conducted linear stability analysis from Ahlers et al. [59]. A very good agreement was also obtained between our results and the estimates occurring from the amplitude equation $[60,64]$ for $\Gamma \geqslant 1$, i.e., for the domain of validity of the amplitude equation; see Eqs. (87) and (88). In the limit $\Gamma \rightarrow \infty$, our estimates of the critical Rayleigh number $\mathrm{Ra}_{c, \Gamma \rightarrow \infty}$ for the onset of convection in confined geometries tend to $(2 \pi)^{4}$, which is about $8.8 \%$ smaller than Jeffreys' 1708 [23]. Note that the Jeffreys result was derived from the linear stability analysis, where the consideration of the perturbations in the horizontal directions was restricted to a single wave of the form $\sim \exp \left(i k_{x} x+i k_{y}+\sigma t\right)$, and, therefore, it approximates the onset of bulk convection only. Our ansatz admits perturbations of any form, under the restrictions that they are represented by combinations of the eigenfunctions that change the sign in the vertical direction and at least in one horizontal direction and that the average of these perturbations over the whole domain vanishes. Therefore, our consideration includes not only the bulk modes but also the wall modes by the onset of convection [see example in Fig. 3 and Figs. 9(e) and 9(f)].

What has been interpreted as "subcritical" modes in some (almost) Oberbeck-Boussinesq Rayleigh-Bénard experiments is nothing else by wall modes (note that subcritical convection does not exist in the Oberbeck-Boussinesq case). The wall modes contribute a little to the total heat transport and therefore are undetectable in the Nusselt number measurements, based on which one recognizes the onset of the subcritical regime. At the same time, the probability that modes with the near the wall activity occur prior to the bulk modes is high in large aspect ratio cylindrical containers. As we have explained, the reason for that is that the eigenvalues of the wall modes are distributed much denser than the eigenvalues of the bulk modes and that the critical Rayleigh number for the onset of convection is directly related to these eigenvalues. Once the wall modes set in, they ease and realize the implementation of the well-known property of the roll-shaped structures to approach the sidewall of the container at right angles.

We have also addressed the question, which shape of the convection cell is optimal, for the given Oberbeck-Boussinesq fluid and given the maximal size of the plates and maximal achievable in temperature difference between the plates. Of course, the optimality of the convection cell can be defined very differently. For example, one might simply want to have a possibly wide container of a fixed sufficiently large height, to study turbulent superstructures [121,157]. In this paper, under the optimal shape of the convection cell, we, however, understand such a shape, which provides the maximal range in Rayleigh numbers (measured in orders of magnitude), from the onset of convection up to the maximal achievable Rayleigh number. In other words, the cell of the optimal shape provides the maximal ratio between the maximal achievable Rayleigh number and the critical Rayleigh number for the onset of convection in the same container. For the case of no-slip boundaries we have calculated the optimal $\Gamma$, Eqs. (114), and showed that deviations from the optimal $\Gamma$ lead to a reduction of the attainable Ra range, as $\log _{10}(\Gamma)$ for $\Gamma \rightarrow 0$ and as $\log _{10}\left(\Gamma^{-3}\right)$ for $\Gamma \rightarrow \infty$. One should note here that in real experiments, the lowest Rayleigh number might be restricted not by the onset of convection, but rather by the smallest reliable temperature difference that one can achieve in the experiment.

Furthermore, we have derived that the relevant length scale in Rayleigh-Bénard convection for any $\Gamma$ is $\ell \sim D / \sqrt{\Gamma^{2}+C}=H / \sqrt{1+C / \Gamma^{2}}$, Eq. (125), and not simply the distance between the plates, $H$. For large $\Gamma \gg 1$ one indeed has $\ell \approx H$, while for small $\Gamma \ll 1$, the diameter of the plates, $D$, determines the relevant length scale, $\ell \approx D$. Moreover, we have shown that the scaling of the reduced heat transport data, i.e., $(\mathrm{Nu}-1) \mathrm{Ra}^{-1 / 3}$, is independent of the container aspect ratio $\Gamma$ if plotted versus the Rayleigh number $\mathrm{Ra}_{\ell}$, which is based on the relevant length scale $\ell$. Using this relevant length scale $\ell$, we managed to put on one master curve plenty of numerical and experimental reduced heat transport data $(\mathrm{Nu}-1) \mathrm{Ra}^{-1 / 3}$, for the aspect ratio of the cylindrical container from $1 / 32$ to 32 (see also [59]).

A further example of the applicability of our ansatz is the derived accurate estimate of the principle range of possible states in two-dimensional turbulent Rayleigh-Bénard convection with 
no-slip plates, in terms of the roll aspect ratio $\Gamma_{r}$, namely, $0.7 \lesssim \Gamma_{r} \lesssim 1.6$, Eq. (161). This estimate does not include any information on the fluid properties, the aspect ratio of the domain, or the Rayleigh number. Independently from these input characteristics the estimate (161) for the roll aspect ratio should hold. Which exactly rolls aspect ratio states the system chooses depends on the balance between the kinetic energy and its dissipation; see also Wang et al. [153].

As we have seen in this study, the lateral confinement of the convection cell can significantly influence all global response characteristics in the system and also the global structure of the convective flow. Therefore it is desired in the future to advance also the boundary-layer theory for Rayleigh-Bénard convection (see Shishkina et al. [165,166] and Ching et al. [167-169]) to the case of confined plates, in order to obtain accurate predictions of the profiles of the main flow characteristics in confined geometries.

Furthermore, the here developed approach, which is based on the analysis of the relevant and most energetic modes in terms of the Laplace eigenfunctions, can be further applied to study formation and dynamics of the superstructures in wide domains [153,158,159], and in general, the dependence of the wall-bounded flows on the geometry of the container in different systems, for example, the Taylor-Couette flows [170], double-diffusive convection [171], modulated with the boundary conditions thermal convection [172,173], thermomagnetohydrodynamic problems [174], internally heated convection [46,175-178], vertical and horizontal convection [179-182], and classical and rotating Rayleigh-Bénard convection, in particular, its wall modes regime in a form of traveling waves [79-81,84,86,88,162], as well as other traveling-wave wall mode regimes that occur in nonrotating convection of fluid mixtures [90].

The problems can have different driving forces, different boundary conditions, and different geometries. Solving the problems with modified steady boundary conditions or modified container geometry seems to be straightforward: here one would need to calculate and examine Laplace eigenmodes for the prescribed boundary conditions in specific geometries. Problems of convection in containers with plate roughness or under the presence of rigid obstacles inside the container or convection in porous media (see, e.g., [183]) are also directly related to the problems of the confined geometry of the container in convective processes. Investigations of these topics might raise additional problems related to the convex shape of the domain occupied with fluid. Interesting and important is also to investigate the systems with dynamically changing driving force, container geometry, and/or boundary conditions, for example, those related to solidification or melting of the working substance (see, e.g., [184]. And finally, how the non-Oberbeck-Boussinesq effects change all convective processes and their main response characteristics, starting from the onset of convection and up to highly turbulent regimes, remains to be one of the challenging problems to investigate.

To sum up: the shape of the Rayleigh-Bénard container matters. Now we know a little more about how the container shape influences the critical Rayleigh numbers and typical flows patterns and length scales. Many questions still remain to be answered.

\section{ACKNOWLEDGMENTS}

I would like to express my sincere gratitude to all my colleagues for all the discussions related to this paper. My special thanks for very useful comments go to E. Bodenschatz, R. E. Ecke, S. Horn, D. Lohse, A. Pumir, P. Reiter, An. Shishkin, C. Sun, S. Weiss, and L. Zwirner. I also want to thank P. Reiter and Al. Shishkin for their help with the figures.

[1] A. Oberbeck, Über die Wärmeleitung der Flüssigkeiten bei Berücksichtigung der Strömungen in Folge von Temperaturdifferenzen, Ann. Phys. (Berlin) 243, 271 (1879).

[2] J. Boussinesq, Théorie analytique de la chaleur (Gauthier-Villars, Paris, 1903).

090502-45 
[3] H. Bénard, Les tourbillons cellulairs dans une nappe liquide, Rev. Gén. Sciences Pure Appl. 11, 1261 (1900).

[4] H. Bénard, Les tourbillons cellulairs dans une nappe liquide.-Méthodes optiques d'observation et d'enregistrement, J. Phys. Théor. Appl. 10, 254 (1901).

[5] Lord Rayleigh, On convection currents in a horizontal layer of fluid, when the higher temperature is on the under side, Philos. Mag.6, 32, 529 (1916).

[6] E. Bodenschatz, W. Pesch, and G. Ahlers, Recent developments in Rayleigh-Bénard convection, Annu. Rev. Fluid Mech. 32, 709 (2000).

[7] G. Ahlers, S. Grossmann, and D. Lohse, Heat transfer and large scale dynamics in turbulent RayleighBénard convection, Rev. Mod. Phys. 81, 503 (2009).

[8] D. Lohse and K.-Q. Xia, Small-scale properties of turbulent Rayleigh-Bénard convection, Annu. Rev. Fluid Mech. 42, 335 (2010).

[9] F. Chillà and J. Schumacher, New perspectives in turbulent Rayleigh-Bénard convection, Eur. Phys. J. E 35, 58 (2012).

[10] H. T. Rossby, On thermal convection driven by non-uniform heating from below: An experimental study, Deep Sea Res. 12, 9 (1965).

[11] G. O. Hughes and R. W. Griffiths, Horizontal convection, Annu. Rev. Fluid Mech. 40, 185 (2008).

[12] G. J. Sheard and M. P. King, Horizontal convection: Effect of aspect ratio on Rayleigh number scaling and stability, Appl. Math. Model. 35, 1647 (2011).

[13] O. Shishkina, S. Grossmann, and D. Lohse, Heat and momentum transport scalings in horizontal convection, Geophys. Res. Lett. 43, 1219 (2016).

[14] O. Shishkina, Mean flow structure in horizontal convection, J. Fluid Mech. 812, 525 (2017).

[15] A. Belmonte, A. Tilgner, and A. Libchaber, Turbulence and internal waves in side-heated convection, Phys. Rev. E 51, 5681 (1995).

[16] H. Yu, N. Li, and R. E. Ecke, Scaling in laminar natural convection in laterally heated cavities: Is turbulence essential in the classical scaling of heat transfer? Phys. Rev. E 76, 026303 (2007).

[17] C. S. Ng, A. Ooi, D. Lohse, and D. Chung, Vertical natural convection: Application of the unifying theory of thermal convection, J. Fluid Mech. 764, 349 (2015).

[18] O. Shishkina, Momentum and heat transport scalings in laminar vertical convection, Phys. Rev. E 93, 051102(R) (2016).

[19] C. S. Ng, A. Ooi, D. Lohse, and D. Chung, Changes in the boundary-layer structure at the edge of the ultimate regime in vertical natural convection, J. Fluid Mech. 825, 550 (2017).

[20] Q. Wang, H. Liu, R. Verzicco, O. Shishkina, and D. Lohse, Regime transitions in thermally driven high-Rayleigh number vertical convection, J. Fluid. Mech. 917, A6 (2021).

[21] A. V. Getling, Rayleigh-Bénard Convection: Structures and Dynamics, edited by R. S. MacKay, Advanced Series in Nonlinear Dynamics, Vol. 11 (World Scientific, 1998).

[22] E. S. C. Ching, Statistics and Scaling in Turbulent Rayleigh-Bénard Convection (Springer, Singapore, 2014).

[23] H. Jeffreys, Some cases of instability in fluid motion, Proc. R. Soc. London Ser. A 118, 195 (1928).

[24] W. H. Reid and D. L. Harris, Some further results on the Bénard problem., Phys. Fluids 1, 102 (1958).

[25] S. Chandrasekhar, Hydrodynamic and Hydromagnetic Stability (Clarendon Press, 1961).

[26] E. L. Koschmieder, Bénard Cells and Taylor Vortices, edited by G. K. Batchelor and L. B. Freund, Cambridge Monographs on Mechanics and Applied Mathematics (Cambridge University Press, 1993).

[27] G. A. Glatzmaier, Introduction to Modeling Convection in Planets and Stars. Magnetic Field, Density Stratification, Rotation (Princeton University Press, 2014).

[28] G. Charlson and R. Sani, Thermoconvective instability in a bounded cylindrical fluid layer, Int. J. Heat Mass Transfer 13, 1479 (1970).

[29] G. Charlson and R. Sani, On thermoconvective instability in a bounded cylindrical fluid layer, Int. J. Heat Mass Transfer 14, 2157 (1971).

[30] I. Catton, Convection in a closed rectangular region: The onset of motion, J. Heat Transfer 92, 186 (1970). 
[31] I. Catton and D. K. Edwards, Initiation of thermal convection in finite right circular cylinders, AlChE J. 16, 594 (1970).

[32] J. C. Buell and I. Catton, The effect of wall conduction on the stability of a fluid in a right circular cylinder heated from below, J. Heat Transf. 105, 255 (1983).

[33] J. Mizushima, Onset of the thermal convection in a finite two-dimensional box, J. Phys. Soc. Jpn. 64, 2420 (1995).

[34] A. Y. Gelfgat, Different modes of Rayleigh-Bénard instability in two- and three-dimensional rectangular enclosures, J. Comput. Phys. 156, 300 (1999).

[35] K. Boronska and L. S. Tuckerman, Standing and travelling waves in cylindrical Rayleigh-Bénard convection, J. Fluid Mech. 559, 279 (2006).

[36] F. Hebert, R. Hufschmid, J. Scheel, and G. Ahlers, Onset of Rayleigh-Bénard convection in cylindrical containers, Phys. Rev. E 81, 046318 (2010).

[37] B. F. Wang, D. J. Ma, C. Chen, and D. J. Sun, Linear stability analysis of cylindrical Rayleigh-Bénard convection, J. Fluid Mech. 711, 27 (2012).

[38] J. Yu, A. Goldfaden, M. Flagstad, and J. D. Scheel, Onset of Rayleigh-Bénard convection for intermediate aspect ratio cylindrical containers, Phys. Fluids 29, 024107 (2017).

[39] G. A. Ostroumov, Free convection under the conditions of the internal problem, Technical Memorandum 1407, National Advisory Committee for Aeronautics (1958).

[40] L. N. Howard, Convection at high Rayleigh number, in Applied Mechanics, edited by H. Görtler (Springer, Berlin, 1966), pp. 1109-1115.

[41] B. I. Shraiman and E. D. Siggia, Heat transport in high-Rayleigh-number convection, Phys. Rev. A 42 , 3650 (1990).

[42] E. Siggia, High Rayleigh number convection, Annu. Rev. Fluid Mech. 26, 137 (1994).

[43] P. J. Schmid and D. S. Henningson, Stability and Transition in Shear Flows (Springer-Verlag, New York, 2001).

[44] L. N. Howard, Heat transport by turbulent convection, J. Fluid Mech. 17, 405 (1963).

[45] D. D. Joseph, Stability of Fluid Motions (Springer Verlag, Berlin, 1976).

[46] D. Goluskin, Internally Heated Convection and Rayleigh-Bénard Convection (Springer, 2016).

[47] O. Reynolds, On the dynamical theory of incompressible viscous fluids and the determination of the criterion, Philos. Trans. R. Soc. London A 186, 123 (1895).

[48] W. M. Orr, The stability or instability of steady motions of a liquid. Part II: A viscous liquid, Proc. R. Irish Acad. (A) 27, 69 (1907).

[49] M. R. Ukhovskii and V. G. Iudovich, On the equation of steady-state convection, Prik. Math. Mek. 27, 353 (1963).

[50] R. L. Sani, On the non-existence of subcritical instability in fluid layers heated from below, J. Fluid Mech. 20, 315 (1964).

[51] D. D. Joseph, On the stability of the Boussinesq equations, Arch. Ration. Mech. Anal. 20, 59 (1965).

[52] D. D. Joseph, Nonlinear stability of the Boussinesq equations by the method of energy, Arch. Ration. Mech. Anal. 22, 163 (1966).

[53] K. Rektorys, Variational Methods in Mathematics, Science and Engineering (D. Reidel, 1977).

[54] D. Pauly, On Maxwell's and Poincaré's constants, Discrete Contin. Dyn. Syst. Series S 8, 607 (2015).

[55] R. Courant and D. Hilbert, Methods of Mathematical Physics (Wiley, New York, 1989).

[56] R. D. Benguria, Isoperimetric inequalities for eigenvalues of the Laplacian, Contemp. Math. 552, 21 (2011).

[57] R. D. Benguria, H. Linde, and B. Loewe, Isoperimetric inequalities for eigenvalues of the Laplacian and the Schrödinger operator, Bull. Math. Sci. 2, 1 (2012).

[58] D. S. Grebenkov and B.-T. Nguyen, Geometrical structure of Laplacian eigenfunctions, SIAM Rev. 55, 601 (2013).

[59] G. Ahlers, E. Bodenschatz, R. Hartmann, X. He, D. Lohse, P. Reiter, R. Stevens, R. Verzicco, M. Wedi, S. Weiss, X. Zhang, L. Zwirner, and O. Shishkina, Aspect ratio dependence of heat transfer in a cylindrical Rayleigh-Bénard cell (unpublished). 
[60] G. Ahlers, M. C. Cross, P. C. Hohenberg, and S. Safran, The amplitude equation near the convective threshold: Application to time-dependent heating experiments, J. Fluid Mech. 110, 297 (1981).

[61] A. Schlueter, D. Lortz, and F. Busse, On the stability of steady finite amplitude convection, J. Fluid Mech. 23, 129 (1965).

[62] A. Newell and J. Whitehead, Finite bandwidth, finite amplitude convection, J. Fluid Mech. 38, 279 (1969).

[63] J. Swift and P. C. Hohenberg, Hydrodynamic fluctuations at the convective instability, Phys. Rev. A 15, 319 (1977).

[64] R. P. Behringer and G. Ahlers, Heat Transport and Temporal Evolution of Fluid Flow near the RayleighBénard Instability in Cylindrical Containers, J. Fluid Mech. 125, 218 (1982).

[65] P. L. Silveston, Wärmedurchgang in waagerechten Flüssigkeitsschichten, Forsch. Ing. Wes. 24, 29 (1958).

[66] V. Croquette, M. Mory, and F. Schosseler, Rayleigh-Bénard convective structures in a cylindrical container, J. Phys. (France) 44, 293 (1983).

[67] K. Stork and U. Möller, Convection in boxes: Experiments, J. Fluid Mech. 54, 599 (1972).

[68] Y. Hu, R. Ecke, and G. Ahlers, Convection near threshold for Prandtl numbers near 1, Phys. Rev. E 48, 4399 (1993).

[69] V. S. Berdnikov and A. G. Kirdyshkin, On the spatial structure of cellular convection, Izv. Acad. Sci. USSR, Atmos. Oceanic Phys. 15, 561 (1979).

[70] C. W. Meyer, G. Ahlers, and D. S. Cannell, Initial Stages of Pattern Formation in Rayleigh-Bénard Convection, Phys. Rev. Lett. 59, 1577 (1987).

[71] K. M. S. Bajaj, N. Mukolobwiez, N. Currier, and G. Ahlers, Wave Number Selection and Large-ScaleFlow Effects Due to a Radial Ramp of the Spacing in Rayleigh-Bénard Convection, Phys. Rev. Lett. 83, 5282 (1999).

[72] G. Ahlers, D. S. Cannell, and V. V. Steinberg, Time Dependence of Flow Patterns Near the Convective Threshold in a Cylindrical Container, Phys. Rev. Lett. 54, 1373 (1985).

[73] E. Bodenschatz, J. R. de Bruyn, G. Ahlers, and D. S. Cannell, Transitions Between Patterns in Thermal Convection, Phys. Rev. Lett. 67, 3078 (1991).

[74] E. Bodenschatz, D. S. Cannell, J. R. de Bruyn, R. Ecke, Y.-C. Hu, K. Lerman, and G. Ahlers, Experiments on three systems with non-variational aspects, Physica D 61, 77 (1992).

[75] V. Steinberg, G. Ahlers, and D. S. Cannell, Pattern formation and wave-number selection by RayleighBénard convection in a cylindrical container, Phys. Scr. T9, 97 (1985).

[76] F. W. J. Olver, Bessel Functions. Part III: Zeros and Associated Values, Royal Society Mathematical Tables, Vol. 7 (Cambridge University Press, Cambridge, 1960).

[77] J. McMahon, On the roots of the Bessel and certain related functions, Ann. Math. 9, 23 (1894).

[78] F. Zhong, R. Ecke, and V. Steinberg, Asymmetric Modes and the Transition to Vortex Structures in Rotating Rayleigh-Bénard Convection, Phys. Rev. Lett. 67, 2473 (1991).

[79] R. Ecke, F. Zhong, and E. Knobloch, Hopf bifurcation with broken reflection symmetry in rotating Rayleigh-Bénard convection, Europhys. Lett. 19, 177 (1992).

[80] F. Zhong, R. Ecke, and V. Steinberg, Rotating Rayleigh-Bénard convection: Asymmetric modes and vortex states, J. Fluid Mech. 249, 135 (1993).

[81] H. F. Goldstein, E. Knobloch, I. Mercader, and M. Net, Convection in a rotating cylinder. Part 1 Linear theory for moderate Prandtl numbers, J. Fluid Mech. 248, 583 (1993).

[82] J. Herrmann and F. H. Busse, Asymptotic theory of wall-attached convection in a rotating fluid layer, J. Fluid Mech. 255, 183 (1993).

[83] K. Zhang and X. Liao, The onset of convection in rotating circular cylinders with experimental boundary conditions, J. Fluid Mech. 622, 63 (2009).

[84] K. Zhang and X. Liao, Theory and Modeling of Rotating Fluids. Convection, Inertial Waves and Precession (Cambridge University Press, 2017).

[85] S. Horn and P. J. Schmid, Prograde, retrograde, and oscillatory modes in rotating Rayleigh-Bénard convection, J. Fluid Mech. 831, 182 (2017). 
[86] B. Favier and E. Knobloch, Robust wall states in rapidly rotating Rayleigh-Bénard convection, J. Fluid Mech. 895, R1 (2020).

[87] X. Zhang, D. P. M. van Gils, S. Horn, M. Wedi, L. Zwirner, G. Ahlers, R. E. Ecke, S. Weiss, E. Bodenschatz, and O. Shishkina, Boundary Zonal Flow in Rotating Turbulent Rayleigh-Bénard Convection, Phys. Rev. Lett. 124, 084505 (2020).

[88] O. Shishkina, Tenacious wall states in thermal convection in rapidly rotating containers, J. Fluid Mech. 898, F1 (2020).

[89] X. Zhang, R. E. Ecke, and O. Shishkina, Boundary zonal flows in rapidly rotating turbulent thermal convection, J. Fluid Mech. 915, A62 (2021).

[90] K. Lerman, G. Ahlers, and D. S. Cannell, Different convection dynamics in mixtures with the same separation ratio, Phys. Rev. E 53, R2041(R) (1996).

[91] S. A. Nazarov, Localization effects for eigenfunctions near to the edge of a thin domain, Mathematica Bohemica 127, 283 (2002).

[92] I. V. Kamotskii and S. A. Nazarov, On eigenfunctions localized in a neighborhood of the lateral surface of a thin domain, J. Math. Sci. 101, 2941 (2000).

[93] G. Cardone, T. Durante, and S. A. Nazarov, The localization effect for eigenfunctions of the mixed boundary value problem in a thin cylinder with distorted ends, SIAM J. Math. Anal. 42, 2581 (2010).

[94] L. Friedlander and M. Solomyak, On the spectrum of the Dirichlet Laplacian in a narrow strip, Israel J. Math. 170, 337 (2009).

[95] L. Friedlander and M. Solomyak, On the spectrum of the Dirichlet Laplacian in a narrow infinite strip, Am. Math. Soc. Transl. 225, 103 (2008).

[96] J. W. S. Rayleigh, The Theory of Sound, Vol. I (Macmillan, 1877).

[97] G. Faber, Beweis, dass unter allen homogenen Membranen von gleicher Fläche und gleicher Spannung die kreisförmige den tiefsten Grundton gibt, in Sitzungberichte der mathematischphysikalischen Klasse der Bayerischen Akademie der Wissenschaften zu München Jahrgang, 169 (1923).

[98] E. Krahn, Über eine von Rayleigh formulierte Minimaleigenschaft des Kreises, Math. Ann. 94, 97 (1925).

[99] G. Szegö, Inequalities for certain eigenvalues of a membrane of given area, J. Rat. Mech. Anal. 3, 343 (1954).

[100] E. Krahn, Über Minimaleigenschaften der Kugel in drei und mehr Dimensionen, Acta Comm. Univ. Tartu (Dorpat) A9, 1 (1926).

[101] A. Henrot and E. Oudet, Minimizing the second eigenvalue of the Laplace operator with Dirichlet boundary conditions, Arch. Ration. Mech. Anal. 169, 73 (2003).

[102] A. Henrot, Isoperimetric inequalities for eigenvalues of the Laplacian, in Geometry of PDEs and Related Problems, edited by R. M. C. Bianchini and A. Henrot, Lecture Notes in Mathematics, Vol. 2220 (Springer, 2018), pp. 47-88.

[103] B. Bogosel, A. Henrot, and I. Lucardesi, Minimization of the eigenvalues of the Dirichlet-Laplacian with a diameter constraint, SIAM J. Math. Anal. 50, 5337 (2018).

[104] S. Wagner and O. Shishkina, Aspect ratio dependency of Rayleigh-Bénard convection in box-shaped containers, Phys. Fluids 25, 085110 (2013).

[105] K. L. Chong, Y. Yang, S.-D. Huang, J.-Q. Zhong, R. J. A. M. Stevens, R. Verzicco, D. Lohse, and K.-Q. Xia, Confined Rayleigh-Bénard, Rotating Rayleigh-Bénard, and Double Diffusive Convection: A Unifying View on Turbulent Transport Enhancement Through Coherent Structure Manipulation, Phys. Rev. Lett. 119, 064501 (2017).

[106] K. L. Chong, S. Wagner, M. Kaczorowski, O. Shishkina, and K.-Q. Xia, Effect of Prandtl number on heat transport enhancement in Rayleigh-Bénard convection under geometrical confinement, Phys. Rev. Fluids 3, 013501 (2018).

[107] L. Zwirner and O. Shishkina, Confined inclined thermal convection in low-Prandtl-number fluids, J. Fluid Mech. 850, 984 (2018).

[108] Y. Hu, R. E. Ecke, and G. Ahlers, Convection under rotation for Prandtl numbers near 1: Linear stability, wave-number selection, and pattern dynamics, Phys. Rev. E 55, 6928 (1997). 
[109] R. Hartmann, K. Chong, R. Stevens, R. Verzicco, and D. Lohse, Heat transport enhancement in confined Rayleigh-Bénard convection feels the shape of the container, Europhys. Lett. (to be published).

[110] A. Nikolaenko, E. Brown, D. Funfschilling, and G. Ahlers, Heat transport by turbulent RayleighBénard convection in cylindrical cells with aspect ratio one and less, J. Fluid Mech. 523, 251 (2005).

[111] S. Weiss and G. Ahlers, Turbulent Rayleigh-Bénard convection in a cylindrical container with aspect ratio $\Gamma=0.50$ and Prandtl number $P r=4.38$, J. Fluid Mech. 676, 5 (2011).

[112] R. J. A. M. Stevens, J. Overkamp, D. Lohse, and H. J. H. Clercx, Effect of aspect ratio on vortex distribution and heat transfer in rotating Rayleigh-Bénard convection, Phys. Rev. E 84, 056313 (2011).

[113] R. J. A. M. Stevens, H. J. H. Clercx, and D. Lohse, Breakdown of the large-scale wind in aspect ratio $\Gamma=1 / 2$ rotating Rayleigh-Bénard flow, Phys. Rev. E 86, 056311 (2012).

[114] D. Funfschilling, E. Brown, A. Nikolaenko, and G. Ahlers, Heat transport by turbulent RayleighBénard convection in cylindrical samples with aspect ratio one and larger, J. Fluid Mech. 536, 145 (2005).

[115] S. Horn and O. Shishkina, Rotating non-Oberbeck-Boussinesq Rayleigh-Bénard convection in water, Phys. Fluids 26, 055111 (2014).

[116] J.-Q. Zhong and G. Ahlers, Heat transport and the large-scale circulation in rotating turbulent RayleighBénard convection, J. Fluid Mech. 665, 300 (2010).

[117] R. Lakkaraju, R. J. A. M. Stevens, R. Verzicco, S. Grossmann, A. Prosperetti, C. Sun, and D. Lohse, Spatial distribution of heat flux and fluctuations in turbulent Rayleigh-Bénard convection, Phys. Rev. E 86, 056315 (2012).

[118] O. Shishkina and A. Thess, Mean temperature profiles in turbulent Rayleigh-Bénard convection of water, J. Fluid Mech. 633, 449 (2009).

[119] O. Shishkina, M. S. Emran, S. Grossmann, and D. Lohse, Scaling relations in large-Prandtl-number natural thermal convection, Phys. Rev. Fluids 2, 103502 (2017).

[120] R. J. A. M. Stevens, D. Lohse, and R. Verzicco, Toward DNS of the ultimate regime of Rayleigh-Bénard convection, Direct Large Eddy Simul. 12, ERCOFTAC Series 27, 215 (2020).

[121] R. J. A. M. Stevens, A. Blass, X. Zhu, R. Verzicco, and D. Lohse, Turbulent thermal superstructures in Rayleigh-Bénard convection, Phys. Rev. Fluids 3, 041501(R) (2018).

[122] X. He, E. Bodenschatz, and G. Ahlers, Universal scaling of temperature variance in Rayleigh-Bénard convection near the transition to the ultimate state, J. Fluid Mech. (to be published).

[123] J. Bailon-Cuba, M. S. Emran, and J. Schumacher, Aspect ratio dependence of heat transfer and largescale flow in turbulent convection, J. Fluid Mech. 655, 152 (2010).

[124] R. J. A. M. Stevens, R. Verzicco, and D. Lohse, Radial boundary layer structure and Nusselt number in turbulent Rayleigh-Bénard convection, J. Fluid Mech. 643, 495 (2010).

[125] M. Wedi, D. P. van Gils, S. Weiss, and E. Bodenschatz, Rotating turbulent thermal convection at very large Rayleigh numbers, J. Fluid Mech. 912, A30 (2021).

[126] G. Ahlers, X. He, D. Funfschilling, and E. Bodenschatz, Heat transport by turbulent Rayleigh-Bénard convection for $\operatorname{Pr} \sim 0.8$ and $3 \times 10^{12} \lesssim R a \lesssim 10^{15}$ : Aspect ratio $\Gamma=0.50$, New J. Phys. 14, 103012 (2012).

[127] X. He, D. Funfschilling, H. Nobach, E. Bodenschatz, and G. Ahlers, Transition to the Ultimate State of Turbulent Rayleigh-Bénard Convection, Phys. Rev. Lett. 108, 024502 (2012).

[128] X. He, D. Funfschilling, H. Nobach, E. Bodenschatz, and G. Ahlers, Comment on Effect of Boundary Layers Asymmetry on Heat Transfer Efficiency in Turbulent Rayleigh-Bénard Convection at Very High Rayleigh Numbers, Phys. Rev. Lett. 110, 199401 (2013).

[129] M. S. Emran and O. Shishkina, Natural convection in cylindrical containers with isothermal ring-shaped obstacles, J. Fluid Mech. 882, A3 (2020).

[130] J. D. Scheel and J. Schumacher, Predicting transition ranges to fully turbulent viscous boundary layers in low Prandtl number convection flows, Phys. Rev. Fluids 2, 123501 (2017).

[131] S. Weiss, P. Wei, and G. Ahlers, Heat-transport enhancement in rotating turbulent Rayleigh-Bénard convection, Phys. Rev. E 93, 043102 (2016). 
[132] G. L. Kooij, M. A. Botchev, E. M. Frederix, B. J. Geurts, S. Horn, D. Lohse, E. P. van der Poel, O. Shishkina, R. J. A. M. Stevens, and R. Verzicco, Comparison of computational codes for direct numerical simulations of turbulent Rayleigh-Bénard convection, Comput. Fluids 166, 1 (2018).

[133] S. Wagner, O. Shishkina, and C. Wagner, Boundary layers and wind in cylindrical Rayleigh-Bénard cells, J. Fluid Mech. 697, 336 (2012).

[134] X. He, D. Funfschilling, E. Bodenschatz, and G. Ahlers, Heat transport by turbulent Rayleigh-Bénard convection for $\operatorname{Pr} \sim 0.8$ and $4 \times 10^{11} \lesssim R a \lesssim 2 \times 10^{14}$ : Ultimate-state transition for aspect ratio $\Gamma=$ 1.00, New J. Phys. 14, 063030 (2012).

[135] O. Shishkina and C. Wagner, Local heat fluxes in turbulent Rayleigh-Bénard convection, Phys. Fluids 19, 085107 (2007).

[136] O. Shishkina and C. Wagner, Analysis of thermal dissipation rates in turbulent Rayleigh-Bénard convection, J. Fluid Mech. 546, 51 (2006).

[137] S. Grossmann and D. Lohse, Scaling in thermal convection: A unifying theory, J. Fluid Mech. 407, 27 (2000).

[138] S. Grossmann and D. Lohse, Thermal Convection for Large Prandtl Numbers, Phys. Rev. Lett. 86, 3316 (2001).

[139] R. J. A. M. Stevens, E. P. van der Poel, S. Grossmann, and D. Lohse, The unifying theory of scaling in thermal convection: The updated prefactors, J. Fluid Mech. 730, 295 (2013).

[140] X. He, E. Bodenschatz, and G. Ahlers, Aspect ratio dependence of the ultimate-state transition in turbulent thermal convection, Proc. Natl. Acad. Sci. USA 117, 30022 (2020).

[141] R. Kraichnan, Turbulent thermal convection at arbitrary Prandtl number, Phys. Fluids 5, 1374 (1962).

[142] E. A. Spiegel, Convection in stars, I. Basic Boussinesq convection, Annu. Rev. Astron. Astrophys. 9, 323 (1971).

[143] S. Grossmann and D. Lohse, Multiple scaling in the ultimate regime of thermal convection, Phys. Fluids 23, 045108 (2011).

[144] E. Bodenschatz, X. He, and D. van Gils, Aspect-ratio dependence of the transition to the ultimate state, 15th European Turbulence Conference, Delft (2015).

[145] X. Chavanne, F. Chilla, B. Castaing, B. Hebral, B. Chabaud, and J. Chaussy, Observation of the Ultimate Regime in Rayleigh-Bénard Convection, Phys. Rev. Lett. 79, 3648 (1997).

[146] X. Chavanne, F. Chillà, B. Chabaud, B. Castaing, and B. Hébral, Turbulent Rayleigh-Bénard convection in gaseous and liquid He, Phys. Fluids 13, 1300 (2001).

[147] P.-E. Roche, F. Gauthier, R. Kaiser, and J. Salort, On the triggering of the ultimate regime of convection, New J. Phys. 12, 085014 (2010).

[148] J. J. Niemela, L. Skrbek, K. R. Sreenivasan, and R. J. Donnely, Turbulent convection at very high Rayleigh numbers, Nature (London) 404, 837 (2000).

[149] J. J. Niemela and K. R. Sreenivasan, Confined turbulent convection, J. Fluid Mech. 481, 355 (2003).

[150] J. J. Niemela and K. R. Sreenivasan, The use of cryogenic helium for classical turbulence: Promises and hurdles, J. Low Temp. Phys. 143, 163 (2006).

[151] P.-E. Roche, The ultimate state of convection: a unifying picture of very high Rayleigh numbers experiments, New J. Phys. 22, 073056 (2020).

[152] E. P. van der Poel, R. J. A. M. Stevens, K. Sugiyama, and D. Lohse, Flow states in two-dimensional Rayleigh-Bénard convection as a function of aspect ratio and Rayleigh number, Phys. Fluids 24, 085104 (2012).

[153] Q. Wang, R. Verzicco, D. Lohse, and O. Shishkina, Multiple States in Turbulent Large-Aspect Ratio Thermal Convection: What Determines the Number of Convection Rolls? Phys. Rev. Lett. 125, 074501 (2020).

[154] Q. Wang, K.-L. Chong, R. J. A. M. Stevens, R. Verzicco, and D. Lohse, From zonal flow to convection rolls in Rayleigh-Bénard convection with free-slip plates, J. Fluid. Mech. 905, A21 (2020).

[155] L. Zwirner, A. Tilgner, and O. Shishkina, Elliptical Instability and Multiple-Roll Flow Modes of the Large-Scale Circulation in Confined Turbulent Rayleigh-Bénard Convection, Phys. Rev. Lett. 125, 054502 (2020). 
[156] P. A. Davidson, Turbulence (Oxford University Press, 2004).

[157] A. Pandey, J. D. Scheel, and J. Schumacher, Turbulent superstructures in Rayleigh-Bénard convection, Nat. Commun. 9, 2118 (2018).

[158] C. C. Lalescu and M. Wilczek, Transitions of turbulent superstructures in generalized Kolmogorov flow, Phys. Rev. Research 3, L022010 (2021).

[159] A. Blass, R. Verzicco, D. Lohse, R. Stevens, and D. Krug, Flow organisation in laterally unconfined Rayleigh-Bénard turbulence, J. Fluid Mech. 906, A26 (2021).

[160] J. W. S. Rayleigh, The problem of the whispering gallery, Philos. Mag. 20, 1001 (1910).

[161] E. Y. Kuo and M. C. Cross, Traveling-wave wall states in rotating Rayleigh-Bénard convection, Phys. Rev. E 47, R2245(R) (1993).

[162] R. E. Ecke, X. Zhang, and O. Shishkina, Connecting wall modes and boundary zonal flows in rotating Rayleigh-Bénard convection, arXiv:2106.07710.

[163] X. M. de Wit, A. J. A. Guzmán, M. Madonia, J. S. Cheng, H. J. H. Clercx, and R. P. J. Kunnen, Turbulent rotating convection confined in a slender cylinder: The sidewall circulation, Phys. Rev. Fluids 5, 023502 (2020).

[164] R. P. J. Kunnen, The geostrophic regime of rapidly rotating turbulent convection, J. Turbulence 22, 267 (2021).

[165] O. Shishkina, S. Horn, S. Wagner, and E. S. C. Ching, Thermal Boundary Layer Equation for Turbulent Rayleigh-Bénard Convection, Phys. Rev. Lett. 114, 114302 (2015).

[166] O. Shishkina, S. Horn, M. S. Emran, and E. S. C. Ching, Mean temperature profiles in turbulent thermal convection, Phys. Rev. Fluids 2, 113502 (2017).

[167] E. S. C. Ching, O.-Y. Dung, and O. Shishkina, Fluctuating thermal boundary layers and heat transfer in turbulent Rayleigh-Bénard convection, J. Stat. Phys. 167, 626 (2017).

[168] E. S. C. Ching, H. S. Leung, L. Zwirner, and O. Shishkina, Velocity and thermal boundary layer equations for turbulent Rayleigh-Bénard convection, Phys. Rev. Research 1, 033037 (2019).

[169] N. C. Tai, E. S. C. Ching, L. Zwirner, and O. Shishkina, Heat flux in turbulent Rayleigh-Bénard convection: Predictions derived from a boundary layer theory, Phys. Rev. Fluids 6, 033501 (2021).

[170] S. Grossmann, D. Lohse, and C. Sun, High-Reynolds number Taylor-Couette turbulence, Annu. Rev. Fluid Mech. 48, 53 (2016).

[171] Y. Yang, R. Verzicco, and D. Lohse, Vertically Bounded Double Diffusive Convection in the Finger Regime: Comparing No-Slip Versus Free-Slip Boundary Conditions, Phys. Rev. Lett. 117, 184501 (2016).

[172] P. Reiter, X. Zhang, R. Stepanov, and O. Shishkina, Generation of zonal flows in convective systems by travelling thermal waves, J. Fluid Mech. 913, A13 (2021).

[173] R. Yang, K. L. Chong, Q. Wang, R. Verzicco, O. Shishkina, and D. Lohse, Periodically Modulated Thermal Convection, Phys. Rev. Lett. 125, 154502 (2020).

[174] O. Zikanov, Y. Listratov, N. Razuvanov, I. Belyaev, P. Frick, and V. Sviridov, Mixed convection in pipe and duct flows with strong magnetic fields, Appl. Mech. Rev. 73, 010801 (2021).

[175] P. H. Roberts, Convection in horizontal layers with internal heat generation. Theory, J. Fluid. Mech. 30, 33 (1967).

[176] D. Goluskin and E. A. Spiegel, Convection driven by internal heating, Phys. Lett. A 377, 83 (2012).

[177] D. Goluskin and E. P. van der Poel, Penetrative internally heated convection in two and three dimensions, J. Fluid Mech. 791, R6 (2016).

[178] Q. Wang, D. Lohse, and O. Shishkina, Scaling in internally heated convection: A unifying theory, Geophys. Res. Lett. 48, e2020GL091198 (2021).

[179] O. Shishkina and S. Wagner, Prandtl-Number Dependence of Heat Transport in Laminar Horizontal Convection, Phys. Rev. Lett. 116, 024302 (2016).

[180] P.-Y. Passaggia, A. Scotti, and B. White, Transition and turbulence in horizontal convection: Linear stability analysis, J. Fluid Mech. 821, 31 (2017).

[181] P. Reiter and O. Shishkina, Classical and symmetrical horizontal convection: Detaching plumes and oscillations, J. Fluid Mech. 892, R1 (2020). 
[182] B. Yan, O. Shishkina, and X. He, Thermal boundary-layer structure in laminar horizontal convection, J. Fluid Mech. 915, R5 (2021).

[183] S. Liu, L. Jiang, K. L. Chong, X. Zhu, Z.-H. Wan, R. Verzicco, R. J. A. M. Stevens, D. Lohse, and C. Sun, From Rayleigh-Bénard convection to porous-media convection: How porosity affects heat transfer and flow structure, J. Fluid Mech. 895, A18 (2020).

[184] Z. Wang, E. Calzavarini, C. Sun, and F. Toschi, How the growth of ice depends on the fluid dynamics underneath, Proc. Natl. Acad. Sci. U. S. A. 118, e2012870118 (2021). 